\title{
Bi-unitary multiperfect numbers, I
}

\section{Pentti Haukkanen $^{1}$ and Varanasi Sitaramaiah ${ }^{2}$}

${ }^{1}$ Faculty of Information Technology and Communication Sciences, FI-33014 Tampere University, Finland e-mail: pentti.haukkanendtuni.fi

2 1/194e, Poola Subbaiah Street, Taluk Office Road, Markapur Prakasam District, Andhra Pradesh, 523316 India e-mail: sitaramaiah52@gmail.com

\begin{abstract}
A divisor $d$ of a positive integer $n$ is called a unitary divisor if $\operatorname{gcd}(d, n / d)=1$; and $d$ is called a bi-unitary divisor of $n$ if the greatest common unitary divisor of $d$ and $n / d$ is unity. The concept of a bi-unitary divisor is due to D. Surynarayana [12]. Let $\sigma^{* *}(n)$ denote the sum of the bi-unitary divisors of $n$. A positive integer $n$ is called a bi-unitary perfect number if $\sigma^{* *}(n)=2 n$. This concept was introduced by C. R. Wall in 1972 [15], and he proved that there are only three bi-unitary perfect numbers, namely 6, 60 and 90.

In 1987, Peter Hagis [6] introduced the notion of bi-unitary multi $k$-perfect numbers as solutions $n$ of the equation $\sigma^{* *}(n)=k n$. A bi-unitary multi 3-perfect number is called a bi-unitary triperfect number. A bi-unitary multiperfect number means a bi-unitary multi $k$-perfect number with $k \geq 3$. Hagis [6] proved that there are no odd bi-unitary multiperfect numbers. We aim to publish a series of papers on bi-unitary multiperfect numbers focusing on multiperfect numbers of the form $n=2^{a} u$, where $u$ is odd. In this paper-part I of the series-we investigate bi-unitary triperfect numbers of the form $n=2^{a} u$, where $1 \leq a \leq 3$. It appears that $n=120=2^{3} 15$ is the only such number. Hagis [6] found by computer the bi-unitary multiperfect numbers less than $10^{7}$. We have found 31 such numbers up to $8.10^{10}$. The first 13 are due to Hagis. After completing this paper we noticed that further numbers are already listed in The On-Line Encyclopedia of Integer Sequences (sequence A189000 Bi-unitary multiperfect numbers). The numbers listed there have been found by direct computer calculations. Our purpose is to present a mathematical search and treatment of bi-unitary multiperfect numbers.
\end{abstract}

Keywords: Perfect numbers, Triperfect numbers, Multiperfect numbers, Bi-unitary analogues. 2010 Mathematics Subject Classification: 11A25. 


\section{Introduction}

Throughout this paper, all lower case letters denote positive integers; $p$ and $q$ denote primes. The letters $u, v$ and $w$ are reserved for odd numbers.

Let $\sigma(n)$ denote the sum of the positive divisors of $n$. It is well known that a positive integer $n$ is called a perfect number if $\sigma(n)=2 n$; a concept introduced by Euclid (300 BC). He demonstrated that numbers of the form $n=2^{p-1}\left(2^{p}-1\right)$ are perfect numbers, where $p$ and $2^{p}-1$ are primes.

The converse that these numbers are the only even perfect numbers was established by Euler two thousand years later. Primes of the form $2^{p}-1$, where $p$ is a prime number, are called Mersenne primes and are denoted by $M_{p}$. Each Mersenne prime gives rise to an even perfect number. The first few examples of even perfect numbers are 6, 28, 496, 8128 and 33550336 which correspond to the primes $p=2,3,5,7$ and 13. One does not know whether this list of even perfect numbers can be continued indefinitely as the answer depends on whether there exist infinitely many Mersenne primes. Till now, 51 Mersenne primes are known (consequently 51 even perfect numbers). The internet information says that the 51 -st Mersenne prime namely $2^{82,589,933}-1$ was found by Patrick Laroche on December 7, 2018. This appears to be the largest known prime with 24,862,048 digits.

Till to date, we do not know an example of an odd perfect number. Euler proved that if $n$ is an odd perfect number, then $n=p^{a} k^{2}$, where $p$ is a prime not dividing the positive integer $k$ and $p \equiv a \equiv 1(\bmod 4)$. In 2012, Pascal Ochem and Michael Rao (cf. [7]) proved that there are no odd perfect numbers less than $10^{1500}$ and an odd perfect number should have at least 101 distinct prime factors. These appear to be the latest results of this type on odd perfect numbers.

A positive integer $n$ is called multiply perfect if $\sigma(n)=k n$ for some positive integer $k$ and the integer $k$ is called the multiplicity of the number $n$; or equivalently $n$ is multiply perfect if and only if $n \mid \sigma(n)$. Multiply perfect numbers with multiplicity $k$ are called multi k-perfect numbers or $k$-fold perfect numbers. Multiply perfect numbers with multiplicity 2 are of course perfect numbers. Multi 3-perfect numbers are called triperfect numbers. For example, the smallest positive integers that are solutions of $\sigma(n)=k n$ for $k=1,2,3,4,5$ are, respectively, $1,6,120,30240$ and $14182439040=2^{7} \cdot 3^{4} \cdot 5 \cdot 7 \cdot 11^{2} 17 \cdot 19$.

Examples of multi $k$-perfect numbers are known for $1 \leq k \leq 11$ (see Wikipedia). The number $n=2^{468} .3^{140} \cdot 5^{66} .11^{40} .17^{11} \cdot 19^{12} \ldots$, a multiply perfect number of multiplicity 11 and with 1907 digits appears to have been found by G.F. Woltman on March 13, 2001.

Till now, the only known odd multiply perfect number is 1 . It is yet to be known whether there are finitely many multiply perfect numbers for each fixed multiplicity $>2$ (see Wikipedia).

An open question is whether each multi $k$-perfect number is divisible by $k !$. Note that L. E. Dickson's book [2] contains a good account of work on multiply perfect numbers (see also [3, 8]).

A divisor $d$ of $n$ is called a unitary divisor if $\operatorname{gcd}(d, n / d)=1$. If $d$ is a unitary divisor of $n$, we write $d \| n$. The concept of unitary divisor was originally due to $\mathrm{R}$. Vaidyanathaswamy [13] who called such a divisor as block factor. The present terminology is due to E. Cohen [1]. Let $\sigma^{*}(n)$ denote the sum of the unitary divisors of $n$. It is known (cf. [1]) that $\sigma^{*}$ is a multiplicative function, $\sigma^{*}(1)=1$ and $\sigma^{*}\left(p^{a}\right)=p^{a}+1$ whenever $p$ is a prime and $a$ is a positive 
integer. M. V. Subbarao and L. J. Warren [11] introduced the notion of a unitary perfect number as follows: a positive integer $n$ is called a unitary perfect number if $\sigma^{*}(n)=2 n$. They proved that there are no odd unitary perfect numbers. The only five unitary perfect numbers known till now are $6,60,90,87360$ and

$$
146361946186458562560000=2^{18} \cdot 3 \cdot 5^{4} \cdot 7 \cdot 11 \cdot 13.19 .37 .79 .109 .157 .313 .
$$

The first four examples were due to M. V. Subbarao and L. J. Warren [11] and the last being due to C. R. Wall $[14,16]$. It is not known whether there exists a unitary perfect number not divisible by 3 (see M. V. Subbarao and L. J. Warren [11]) and also whether there are infinitely many unitary perfect numbers (see M. V. Subbarao [10]).

A positive integer $m$ is called unitary multiply perfect if $m \mid \sigma^{*}(m)$; that is, it is solution of $\sigma^{*}(m)=k m$ for some positive integer $k$. In the case $k>2$, no example of a unitary multiply perfect number is known till now. It is known that if such a number exists, it must be even and greater than $10^{102}$ and must have more than forty four odd prime factors (see $\left.[4,5]\right)$. This problem is probably very difficult to settle.

It is easily seen that if $p$ is a prime, $a$ and $n$ are positive integers such that

$$
p^{a} \mid \sigma^{*}(n) \text { and } n \mid \sigma^{*}\left(p^{a}\right) \text {, }
$$

then

$$
m=p^{a} n
$$

is unitary multiply perfect number. However it has been proved in [9] that the only solutions of (1.1) are $p=2, a=1, n=3 ; p=3, a=1, n=2$; and $p=3, a=2, n=10$. The only solutions of (1.1) in the form (1.2) are $m=6$ and $m=90$, which are unitary perfect numbers. Thus if $k>3$, there are no solutions to the equation $\sigma^{*}(m)=k m$ of the form (1.2).

A divisor $d$ of $n$ is called a bi-unitary divisor if $(d, n / d)^{* *}=1$, where the symbol $(a, b)^{* *}$ denotes the greatest common unitary divisor of $a$ and $b$. The concept of a bi-unitary divisor is due to D. Suryanarayana [12].

Let $\sigma^{* *}(n)$ denote the sum of bi-unitary divisors of $n$. The function $\sigma^{* *}(n)$ is multiplicative, that is, $\sigma^{* *}(1)=1$ and $\sigma^{* *}(m n)=\sigma^{* *}(m) \sigma^{* *}(n)$ whenever $(m, n)=1$. If $p^{\alpha}$ is a prime power and $\alpha$ is odd then every divisor of $p^{\alpha}$ is a bi-unitary divisor; if $\alpha$ is even, each divisor of $p^{\alpha}$ is a bi-unitary divisor except for $p^{\alpha / 2}$. Hence

$$
\sigma^{* *}\left(p^{\alpha}\right)= \begin{cases}\sigma\left(p^{\alpha}\right)=\frac{p^{\alpha+1}-1}{p-1} & \text { if } \alpha \text { is odd, } \\ \sigma\left(p^{\alpha}\right)-p^{\alpha / 2} & \text { if } \alpha \text { is even. }\end{cases}
$$

If $\alpha$ is even, say $\alpha=2 k$, then $\sigma^{* *}\left(p^{\alpha}\right)$ can be simplified to

$$
\sigma^{* *}\left(p^{\alpha}\right)=\left(\frac{p^{k}-1}{p-1}\right) \cdot\left(p^{k+1}+1\right)
$$

From (1.3), it is not difficult to observe that $\sigma^{* *}(n)$ is odd only when $n=1$ or $n=2^{\alpha}$.

The concept of a bi-unitary perfect number was introduced by C. R. Wall in 1972 [15]; a positive integer $n$ is called a bi-unitary perfect number if $\sigma^{* *}(n)=2 n$. In [15], C. R. Wall proved that there are only three bi-unitary perfect numbers, namely 6,60 and 90. 
In 1987, Peter Hagis [6] introduced the notion of bi-unitary multi $k$-perfect numbers; $n$ is called a bi-unitary multi $k$-perfect number if $\sigma^{* *}(n)=k n$. A bi-unitary multiply perfect number is simply a bi-unitary multi $k$-perfect number for some positive integer $k$. Equivalently, bi-unitary multiply perfect numbers $n$ are solutions of $n \mid \sigma^{* *}(n)$. A bi-unitary multi 3-perfect number is called a bi-unitary triperfect number.

Since $\sigma^{* *}\left(p^{\alpha}\right)=\sigma\left(p^{\alpha}\right)$ when $\alpha$ is odd, it follows that if $n=\prod_{i} p_{i}^{\alpha_{i}}$ is the canonical representation of $n$ with each $\alpha_{i}$ odd then $\sigma^{* *}(n)=\sigma(n)$. Hence, if $n$ has all odd exponents in its canonical representation, then $n$ is multiply perfect if and only if it is bi-unitary multiply perfect. We may also note that if $k$ and $n$ are relatively prime and $n$ is bi-unitary multi $k$-perfect, then $k n$ is bi-unitary multi $m$-perfect, where $m=\sigma^{* *}(k)$.

Hereafter, a bi-unitary multiperfect number means a bi-unitary multi $k$-perfect number with $k \geq 3$. Peter Hagis [6] proved that there are no odd bi-unitary multiperfect numbers. This motivates us to examine even bi-unitary multiperfect numbers.

We are going to publish a series of papers on bi-unitary multiperfect numbers focusing on multiperfect numbers of the form $n=2^{a} u$, where $u$ is odd. In this paper, that is the first part of the series, we investigate bi-unitary triperfect numbers of the form $n=2^{a} u$, where $1 \leq a \leq 3$. In Section 3, we prove (see Theorem 3.1) that if $1 \leq a \leq 3$ and $n=2^{a} u$ is a bi-unitary triperfect number, then $a=3$ and $n=120=2^{3} .3 .5$. This shows that there exists only one bi-unitary triperfect number of this type.

Peter Hagis [6] found by computer all bi-unitary multiperfect numbers less than $10^{7}$. We have found these numbers from $10^{7}$ to $8.10^{10}$. A list of these numbers (up to $8.10^{10}$ ) is given in Appendix A. This list contains 31 bi-unitary multiperfect numbers. The first 13 are due to Hagis [6].

Note. After completing this paper we found that even more bi-unitary multiperfect numbers are already listed in The On-Line Encyclopedia of Integer Sequences (sequence A189000 Bi-unitary multiperfect numbers). The numbers listed there have been found by direct computer calculations. On the contrary, our purpose is to present a mathematical search and treatment of bi-unitary multiperfect numbers.

Section 2 contains preliminaries needed in the main topic. Appendix A contains our list of bi-unitary multiperfect numbers less than $8 \cdot 10^{10}$. Appendix B/Appendix $\mathrm{C}$ contain tables helpful in finding the divisibility of $\left(5^{t}-1\right.$ and $\left.5^{t}+1\right) /\left(7^{t}-1\right.$ and $\left.7^{t}+1\right)$ by primes in the interval $[3,2501]$. Appendices D and E contain factorizations of numbers of the form $5^{t}-1$ and $5^{t}+1$, respectively, and Appendices $\mathrm{F}$ and $\mathrm{G}$ contain factorizations of numbers of the form $7^{t}-1$ and $7^{t}+1$, respectively. These are needed here and in the future papers.

\section{Preliminaries}

Lemma 2.1. (I) If $\alpha$ is odd, then

$$
\frac{\sigma^{* *}\left(p^{\alpha}\right)}{p^{\alpha}}>\frac{\sigma^{* *}\left(p^{\alpha+1}\right)}{p^{\alpha+1}}
$$

for any prime $p$. 
(II) For any $\alpha \geq 2 \ell-1$ and any prime $p$,

$$
\frac{\sigma^{* *}\left(p^{\alpha}\right)}{p^{\alpha}} \geq\left(\frac{1}{p-1}\right)\left(p-\frac{1}{p^{2 \ell}}\right)-\frac{1}{p^{\ell}}=\frac{1}{p^{2 \ell}}\left(\frac{p^{2 \ell+1}-1}{p-1}-p^{\ell}\right) .
$$

(III) If $p$ is any prime and $\alpha$ is a positive integer, then

$$
\frac{\sigma^{* *}\left(p^{\alpha}\right)}{p^{\alpha}}<\frac{p}{p-1}
$$

Proof. (I) Since $\alpha$ is odd,

$$
\sigma^{* *}\left(p^{\alpha}\right)=\sigma\left(p^{\alpha}\right)=1+p+p^{2}+\cdots+p^{\alpha}
$$

and

$$
\sigma^{* *}\left(p^{\alpha+1}\right)=\sigma\left(p^{\alpha+1}\right)-p^{\frac{\alpha+1}{2}}=1+p+\cdots+p^{\alpha}+p^{\alpha+1}-p^{\frac{\alpha+1}{2}} .
$$

Hence

$$
\frac{\sigma^{* *}\left(p^{\alpha}\right)}{p^{\alpha}}>\frac{\sigma^{* *}\left(p^{\alpha+1}\right)}{p^{\alpha+1}}
$$

if and only if $p \sigma^{* *}\left(p^{\alpha}\right)>\sigma^{* *}\left(p^{\alpha+1}\right)$

if and only if $p\left[1+p+p^{2}+\cdots+p^{\alpha}\right]>\left[1+p+\cdots+p^{\alpha}+p^{\alpha+1}-p^{\frac{\alpha+1}{2}}\right]$

if and only if $\left[p+p^{2}+\cdots+p^{\alpha+1}\right]>\left[1+p+\cdots+p^{\alpha}+p^{\alpha+1}-p^{\frac{\alpha+1}{2}}\right]$

if and only if $0>1-p^{\frac{\alpha+1}{2}}$.

(II) Let $\alpha \geq 2 \ell-1$.

Case 1. Let $\alpha$ be odd. If $\alpha+1=2 k$, then $k \geq \ell$. By (I),

$$
\begin{aligned}
\frac{\sigma^{* *}\left(p^{\alpha}\right)}{p^{\alpha}} & >\frac{\sigma^{* *}\left(p^{\alpha+1}\right)}{p^{\alpha+1}} \\
& =\frac{\sigma\left(p^{2 k}\right)-p^{k}}{p^{2 k}}=\frac{\left(\frac{p^{2 k+1}-1}{p-1}-p^{k}\right)}{p^{2 k}}=\left(\frac{1}{p-1}\right)\left(p-\frac{1}{p^{2 k}}\right)-\frac{1}{p^{k}} \\
& \geq\left(\frac{1}{p-1}\right)\left(p-\frac{1}{p^{2 \ell}}\right)-\frac{1}{p^{\ell}} .
\end{aligned}
$$

Case 2. Let $\alpha$ be even and $\alpha=2 k$. Then $\alpha \geq 2 \ell-1$ implies that $k \geq \ell$. Hence

$$
\frac{\sigma^{* *}\left(p^{\alpha}\right)}{p^{\alpha}}=\left(\frac{1}{p-1}\right)\left(p-\frac{1}{p^{2 k}}\right)-\frac{1}{p^{k}} \geq\left(\frac{1}{p-1}\right)\left(p-\frac{1}{p^{2 \ell}}\right)-\frac{1}{p^{\ell}} .
$$

The proof of (II) is complete.

(III) We have

$$
\frac{\sigma^{* *}\left(p^{\alpha}\right)}{p^{\alpha}} \leq \frac{\sigma\left(p^{\alpha}\right)}{p^{\alpha}}=\frac{p^{\alpha+1}-1}{p^{\alpha}(p-1)}=\frac{1}{p-1} \cdot\left[p-\frac{1}{p^{\alpha}}\right]<\frac{p}{p-1}
$$

Remark 2.1. (I) and (III) of Lemma 2.1 are mentioned in C. R. Wall [15]; (II) of Lemma 2.1 has been used by him [15] without explicitly stating it.

Lemma 2.2. Let $a>1$ be an integer not divisible by an odd prime $p$ and let $\alpha$ be a positive integer. Let $r$ denote the least positive integer such that $a^{r} \equiv 1\left(\bmod p^{\alpha}\right) ;$ then $r$ is usually denoted by $\operatorname{ord}_{p^{\alpha}}$ a. We have the following properties: 
(i) If $r$ is even, then $s=r / 2$ is the least positive integer such that $a^{s} \equiv-1\left(\bmod p^{\alpha}\right)$. Also, $a^{t} \equiv-1\left(\bmod p^{\alpha}\right)$ for a positive integer $t$ if and only if $t=s u$, where $u$ is odd.

(ii) If $r$ is odd, then $p^{\alpha} \nmid a^{t}+1$ for any positive integer $t$.

The proof is straightforward.

Remark 2.2. Let $a, p, r$ and $s=r / 2$ be as in Lemma $2.2(\alpha=1)$. Then, $p \mid a^{t}-1$ if and only if $r \mid t$. If $t$ is odd and $r$ is even, then $r \nmid t$. Hence, $p \nmid a^{t}-1$. Also, $p \mid a^{t}+1$ if and only if $t=s u$, where $u$ is odd. In particular, if $t$ is even and $s$ is odd, then $p \nmid a^{t}+1$. In order to check the divisibility of $a^{t}-1$ (when $t$ is odd) by an odd prime $p$, we can confine to those $p$ for which $\operatorname{ord}_{p} a$ is odd. Similarly, for examining the divisibility of $a^{t}+1$ by $p$ when $t$ is even we need to consider primes $p$ with $s=\operatorname{ord}_{p} a / 2$ even.

Lemma 2.3. Let $k$ be odd and $k \geq 3$. Let $p \neq 5$.

(a) If $p \in[3,2520]-\{11,19,31,71,181,829,1741\}$, ord ${ }_{p} 5$ is odd and $p \mid 5^{k}-1$, then we can find a prime $p^{\prime}$ (depending on $p$ ) such that $p^{\prime} \mid \frac{5^{k}-1}{4}$ and $p^{\prime} \geq 2521$.

(b) If $q \in[3,2520]-\{13,313,601\}, s=\frac{1}{2} \operatorname{ord}_{q} 5$ is even and $q \mid 5^{k+1}+1$ then we can find $a$ prime $q^{\prime}$ (depending on $q$ ) such that $q^{\prime} \mid \frac{5^{k+1}+1}{2}$ and $q^{\prime} \geq 2521$.

Proof. (a) Let $p \mid 5^{k}-1$. If $r=\operatorname{ord}_{p} 5$, that is, $r$ is the least positive integer such that $5^{r} \equiv 1(\bmod p)$, then $r \mid k$. Since $k$ is odd, $r$ must be odd. Also, $5^{r}-1 \mid 5^{k}-1$. Let

$$
S_{5}=\left\{(p, r): p \neq 5, p \in[3,2520] \text { and } r=\operatorname{ord}_{p} 5 \text { odd }\right\}
$$

From Appendix B, we have

$S_{5}=\{(11,5),(19,9),(31,3),(59,29),(71,5),(79,39),(101,25),(109,27),(131,65),(139,69)$,

$(149,37),(151,75),(179,89),(181,15),(191,19),(199,33),(211,35),(239,119)$,

$(251,25),(269,67),(271,27),(311,155),(331,165),(359,179),(379,21),(389,97)$,

$(401,25),(409,17),(419,209),(431,215)(439,219),(461,115),(479,239),(491,245)$,

$(499,249),(541,135),(569,71),(571,285),(599,299),(619,309)(631,35),(659,329)$,

$(691,115),(719,359),(739,123),(751,375),(811,405),(829,9),(839,419),(859,429)$,

$(911,455),(919,459),(941,235),(971,485),(991,495),(1019,509),(1021,255)$,

(1031, 515), (1039, 173), (1051, 525), (1061, 265), (1069, 267), (1091, 109), (1151, 575),

$(1171,45),(1231,615),(1259,629),(1279,639),(1291,215),(1319,659),(1399,699)$,

(1429, 119), (1439, 719), (1451, 725), (1459, 243), (1471, 735), (1499, 749), (1511, 755),

$(1531,85),(1559,779),(1571,785),(1579,789),(1609,67),(1619,809),(1621,405)$,

$(1699,283),(1741,15),(1759,293),(1811,905),(1831,305),(1861,31),(1871,935)$,

(1879, 939), (1931, 965), (1949, 487), (1951, 975), (1979, 989), (1999, 999), (2011, 1005),

(2039, 1019), (2099, 1049), (2111, 1055), (2131, 1065), (2179, 1089), (2251, 1125),

$(2239,373),(2269,567),(2309,577),(2311,165),(2339,1169),(2351,1175),(2371,1185)$,

$(2239,373),(2399,1199),(2411,1205),(2441,305),(2459,1229)\}$. 
Let $p \mid 5^{k}-1$ and $p \in[3,2520]-\{11,19,31,71,181,829,1741\}$. Then

$$
(p, r) \in S_{5}-\{(11,5),(19,9),(31,3),(71,5),(181,15),(829,9),(1741,15)\},
$$

where $r=\operatorname{ord}_{p} 5$. Also, $5^{r}-1 \mid 5^{k}-1$. To prove (a), it is enough to show that $\frac{5^{r}-1}{4}$ is divisible by a prime $p^{\prime} \geq 2521$. From Appendix D, we can know the factors of $5^{r}-1$. By examining the factors of $5^{r}-1$ for $r \notin\{5,9,3,5,15,9,15\}$ which correspond to the primes $11,19,31,71,181,829$ and 1741 , respectively, we infer that we can a find a prime $p^{\prime}\left|\frac{5^{r}-1}{4}\right| \frac{5^{k}-1}{4}$ satisfying $p^{\prime} \geq 2521$. This proves (a).

For example, if $p=59$, then $r=29$. From Appendix D,

$$
5^{29}-1=\{\{2,2\},\{59,1\},\{35671,1\},\{22125996444329,1\}\} .
$$

Thus, if $59 \mid 5^{k}-1$, then $p^{\prime}=35671 \mid \frac{5^{k}-1}{4}$ and trivially $p^{\prime}>2521$.

(b) Let $q \mid 5^{k+1}+1$ and $q \in[3,2520]-\{13,313,601\}$. Let $r=\operatorname{ord}_{q} 5$. If $r$ is odd, then $q \nmid 5^{k+1}+1$ (See Remark $2.2(a=5)$ ). We may assume that $r$ is even. Let $s=r / 2$. Then $s$ is the least positive integer such that $q \mid 5^{s}+1$. Again from Remark $2.2(a=5), q \nmid 5^{k+1}+1$ if $s$ is odd. Since $q \mid 5^{k+1}+1$, we have that $s$ is even. Also, $k+1=s u$, where $u$ is odd. This implies that $5^{s}+1 \mid 5^{k+1}+1$. Let

$$
T_{5}=\left\{(q, s): q \neq 5, q \in[3,2520] \text { and } s=\frac{1}{2} \operatorname{ord}_{q} 5 \text { even }\right\} .
$$

From Appendix B, we have

$T_{5}=\{(13,2),(17,8),(37,18),(41,10),(53,26),(73,36),(89,22),(97,48),(113,56),(137,68)$, $(157,78),(173,86),(193,96),(197,98),(233,116),(241,20),(257,128),(277,138)$, $(281,70),(293,146),(313,4),(317,158),(337,56),(353,176),(373,186),(397,198)$, $(433,216),(457,76),(557,278),(577,288),(593,296),(601,6),(613,306),(617,308)$, $(641,32),(653,326),(673,336),(677,338),(733,122),(757,378),(769,64),(773,386)$, $(797,398),(809,202),(853,142),(857,428),(877,438),(881,220),(929,116),(937,468)$, $(953,476),(977,488),(997,166),(1009,252),(1013,252),(1033,506),(1049,262)$, $(1093,546),(1097,548),(1117,186),(1153,576),(1193,596),(1201,300),(1213,606)$, $(1217,608),(1237,618),(1249,312),(1277,638),(1289,322),(1297,72),(1321,330)$, $(1361,170),(1373,686),(1409,352),(1433,716),(1453,66),(1481,370),(1489,186)$, (1493, 746), (1553, 776), (1597, 266), (1601, 200), (1613, 806), (1637, 818), (1657, 276), (1693, 846), (1697, 848), (1733, 866), (1753, 292), (1777, 888), (1801, 450), (1873, 312), $(1877,938),(1889,236)(1913,956),(1933,966),(1973,34),(1993,996),(1997,998)$, $(2017,1008),(2053,1026),(2081,260),(2089,522),(2113,1056),(2137,356)$, $(2153,1076),(2161,540),(2213,1106),(2237,1118),(2273,1136),(2281,30)$, (2293, 1146), (2297, 1148), (2333, 1166), (2357, 1178), (2377, 1188), (2393, 1196), (2417, 1208), (2473, 1236), (2477, 1238). 
Let $q \mid 5^{k+1}+1$ and $q \in[3,2520]-\{13,313,601\}$. Then $(q, s) \in T-\{(13,2),(313,4),(601,6)\}$, where $s=\frac{1}{2} \operatorname{ord}_{q} 5$. To prove (b), it is enough to show that $\frac{5^{s}+1}{2}$ is divisible by a prime $q^{\prime} \geq 2521$ for all $s \in T^{\prime}=\{s:(q, s) \in T-\{(13,2),(313,4),(601,6)\}\}$. This follows by examining the factors of $5^{t}+1$ given in Appendix E.

For example, if $q=53$, then $s=26$. Also,

$$
5^{26}+1=\{\{2,1\},\{13,2\},\{53,1\},\{83181652304609,1\}\} .
$$

We can take $q^{\prime}=83181652304609$.

The proof of Lemma 2.3 is complete.

Lemma 2.4. Let $k$ be odd and $k \geq 3$. Let $p \neq 7$.

(a) If $p \in[3,2520]-\{3,19,37,1063\}, r=\operatorname{ord}_{p} 7$ is odd and $p \mid 7^{k}-1$, then we can find a prime $p^{\prime}$ (depending on $p$ ) such that $p^{\prime} \mid \frac{7^{k}-1}{6}$ and $p^{\prime} \geq 2521$.

(b) If $q \in[3,1193]-\{5,13,181,193,409\}, s=\frac{1}{2} \operatorname{ord}_{q} 7$ is even and $q \mid 7^{k+1}+1$, then we can find a prime $q^{\prime}$ (depending on $q$ ) such that $q^{\prime} \mid \frac{7^{k+1}+1}{2}$ and $q^{\prime}>1193$.

Proof. (a) Let $p \mid 7^{k}-1$. If $r=\operatorname{ord}_{p} 7$ that is, $r$ is the least positive integer such that $7^{r} \equiv 1(\bmod p)$, then $r \mid k$. Since $k$ is odd, $r$ must be odd. Also, $7^{r}-1 \mid 7^{k}-1$. Let

$$
S_{7}=\left\{(p, r): p \neq 7, p \in[3,2520] \text { and } r=\operatorname{ord}_{p} 7 \text { odd }\right\} .
$$

From Appendix C, we have

$S_{7}=\{(3,1),(19,3),(29,7),(31,15),(37,9),(47,23),(59,29),(83,41),(103,51),(109,27)$, $(131,65),(139,69),(167,83),(199,99),(223,37),(227,113),(251,125),(271,135)$, $(283,141),(307,153),(311,31),(367,61),(383,191),(389,97),(419,19),(439,73)$, $(467,233),(479,239),(503,251),(523,261),(563,281),(587,293),(607,101),(613,153)$, $(619,309),(643,321),(647,323),(653,163),(691,345),(701,175),(709,177),(719,359)$, $(727,363),(757,189),(787,393),(809,101),(811,27),(839,419),(859,(429),(877,219)$, $(887,443),(971,97),(983,491),(1039,519),(1061,265),(1063,9),(1091,545),(1093,273)$, $(1123,11),(1151,115),(1213,303),(1223,611),(1231,615),(1259,629),(1279,639)$, (1291, 645), (1307, 653), (1319, 659), (1373, 343), (1381, 345), (1399, 699), (1427, 713), $(1429,357),(1447,241),(1453,121),(1459,243),(1481,185),(1483,741),(1487,743)$, $(1511,755),(1531,85),(1543,257),(1559,779),(1567,783),(1571,785),(1621,81)$, $(1627,813),(1699,849),(1733,433),(1783,891),(1811,181),(1823,911),(1847,923)$, (1867, 933), (1873, 117), (1879, 939), (1907, 953), (1931, 965), (1951, 975), (1979, 989), (1987, 993), (1997, 499), (2063, 1031), (2069, 517), (2099, 517), (2131, 355), (2153, 269), $(2203,1101),(2213,553),(2237,559),(2239,373),(2243,1121),(2267,1133),(2269,567)$, $(2287,1143),(2333,583),(2351,1175),(2371,237),(2377,27),(2383,397),(2399,1199)$, $(2411,1205),(2467,1233)\}$. 
Let $p \mid 7^{k}-1$ and $p \in[3,2520]-\{3,19,37,1063\}$. Then

$$
(p, r) \in S_{7}-\{(3,1),(19,3),(37,9),(1063,9)\},
$$

where $r=\operatorname{ord}_{p} 7$. Also, $7^{r}-1 \mid 7^{k}-1$. To prove (a), it is enough to show that $\frac{7^{r}-1}{6}$ is divisible by a prime $p^{\prime} \geq 2521$. From Appendix F, we know the factors of $7^{r}-1$. By examining the factors of $7^{r}-1$ for $r \notin\{1,3,9,9\}$ which correspond to the primes $3,19,37,1063$, respectively, we infer that we can a find a prime $p^{\prime}\left|\frac{7^{r}-1}{6}\right| \frac{7^{k}-1}{6}$ satisfying $p^{\prime} \geq 2521$. This proves (a).

For example, if $p=47$, then $r=23$. Also,

$$
7^{23}-1=\{\{2,1\},\{3,1\},\{47,1\},\{3083,1\},\{31479823396757,1\}\} .
$$

We can take $p^{\prime}=3083$.

(b) Let $q \mid 7^{k+1}+1$ and $q \in[3,1193]-\{5,13,181,193,409\}$. Let $r=\operatorname{ord}_{q} 7$. If $r$ is odd, then $q \nmid 7^{k+1}+1$ (See Remark $2.2(a=7)$ ). We may assume that $r$ is even. Let $s=r / 2$. Then $s$ is the least positive integer such that $q \mid 7^{s}+1$. Again from Remark $2.2(a=7), q \nmid 7^{k+1}+1$ if $s$ is odd. Since $q \mid 7^{k+1}+1$, we have that $s$ is even. Also, $k+1=s u$, where $u$ is odd. This implies that $7^{s}+1 \mid 7^{k+1}+1$. Let

$$
T_{7}=\left\{(q, s): q \neq 7, q \in[3,1193] \text { and } s=\frac{1}{2} \operatorname{ord}_{q} 7 \text { even }\right\} .
$$

From Appendix C, we have

$$
\begin{aligned}
T_{7}= & (5,2),(13,6),(17,8),(41,20),(61,30),(73,12),(89,44),(97,48),(101,50),(137,34), \\
& (157,26),(173,86),(181,6),(193,12)(229,114),(233,58),(241,120),(257,128), \\
& (269,134),(281,10),(293,146),(313,52),(337,28),(349,174),(353,16),(397,198), \\
& (401,100),(409,12),(433,216),(449,56),(461,230),(509,254),(521,260),(569,142), \\
& (577,288),(593,296),(601,300),(617,154),(641,160),(661,22),(673,56),(677,38), \\
& (733,366),(761,380),(769,128),(773,386),(797,398),(829,138),(853,142),(857,428), \\
& (881,40),(929,464),(937,468),(941,470),(977,244),(997,498),(1009,126), \\
& (1013,506),(1021,170),(1033,86),(1049,524),(1069,534),(1097,548),(1109,554), \\
& (1129,282),(1153,192),(1181,590),(1193,596)\} .
\end{aligned}
$$

Let $q \mid 7^{k+1}+1$ and $q \in[3,1193]-\{5,13,181,193,409\}$. Then

$$
(q, s) \in T_{7}-\{(5,2),(13,6),(181,6),(193,12),(409,12)\},
$$

where $s=\frac{1}{2} \operatorname{ord}_{q} 7$. To prove (b), it is enough to show that $\frac{7^{s}+1}{2}$ is divisible by a prime $q^{\prime}>1193$ for all $s \in T_{7}^{\prime}=\left\{s:(q, s) \in T_{7}-\{(5,2),(13,6),(181,6),(193,12),(409,12)\}\right.$. This follows by examining the factors of $7^{s}+1$ given in Appendix G.

For example if $q=41$ then $s=20$. Also,

$$
7^{20}+1=\{\{2,1\},\{41,1\},\{1201,1\},\{810221830361,1\}\} .
$$

We can take $q^{\prime}=1201$. 


\section{Bi-unitary triperfect numbers of the form $n=2^{a} u$ \\ with $1 \leq a \leq 3$}

In this section, we find all bi-unitary triperfect numbers $n$ such that $2^{a} \| n$, where $1 \leq a \leq 3$.

Theorem 3.1. Let $1 \leq a \leq 3$. If $n=2^{a} u$ (where $u$ is odd) is a bi-unitary triperfect number, then $a=3$ and $n=120=2^{3} .3 .5$.

Proof. Let $n=2^{a} u$ be a bi-unitary triperfect number so that $n$ is a solution of

$$
\sigma^{* *}(n)=3 n
$$

(i) Let $a=1$. Then $n=2 u$. From (3.1), we have $6 u=3 n=\sigma^{* *}(2 u)=\sigma^{* *}(2) \cdot \sigma^{* *}(u)=3 \sigma^{* *}(u)$ so that $\sigma^{* *}(u)=2 u$. Hence $u$ is an odd bi-unitary perfect number. But such a number does not exist (C. R. Wall [15]). Hence $a=1$ is not possible.

(ii) Let $a=2$ so that $n=4 u$. From (3.1), we have

$$
12 u=3 n=\sigma^{* *}(n)=\sigma^{* *}(4) \cdot \sigma^{* *}(u)=5 \cdot \sigma^{* *}(u) .
$$

and so $5 \mid u$. Hence we can write $u=5^{b} . v$, where $b \geq 1$ and $v$ is prime to 2.5 . Thus we have

$$
n=4 u=2^{2} \cdot 5^{b} \cdot v, \quad(v, 2.5)=1,
$$

and from (3.1a), we obtain $12.5^{b} \cdot v=5 \cdot \sigma^{* *}\left(5^{b}\right) \cdot \sigma^{* *}(v)$, or

$$
2^{2} \cdot 3 \cdot 5^{b-1} \cdot v=\sigma^{* *}\left(5^{b}\right) \cdot \sigma^{* *}(v) .
$$

Considering the parity of the values of the function $\sigma^{* *}$ in (3.1c), it is clear that $v$ cannot have more than one odd prime factor. Hence $v=1$ or $v=p^{c}$, where $p$ is an odd prime. In any case $\frac{\sigma^{* *}(v)}{v}<\frac{p}{p-1}$. Hence from (3.1b), we have by Lemma 2.1,

$$
3=\frac{\sigma^{* *}(n)}{n}<\frac{5}{4} \cdot \frac{5}{4} \cdot \frac{p}{p-1} \leq \frac{5}{4} \cdot \frac{5}{4} \cdot \frac{3}{2}=2.34375<3,
$$

a contradiction. Thus $a=2$ is not possible.

(iii) Let $a=3$, so that $n=2^{3} \cdot u$. Since $\sigma^{* *}\left(2^{3}\right)=15$, from (3.1), we obtain $3.2^{3} \cdot u=3 n=$ $\sigma^{* *}(n)=15 \cdot \sigma^{* *}(u)$ and after simplification,

$$
2^{3} \cdot u=5 \cdot \sigma^{* *}(u) .
$$

From (3.2) we have $5 \mid u$ and so $u=5^{b} . v$, where $(v, 2.5)=1$. Hence

$$
n=2^{3} \cdot 5^{b} \cdot v
$$

and from (3.2), after simplification we obtain

$$
2^{3} \cdot 5^{b-1} \cdot v=\sigma^{* *}\left(5^{b}\right) \cdot \sigma^{* *}(v)
$$

$v$ cannot have more than two odd prime factors.

The remaining proof of (iii) depends on the following lemmas: 
Lemma 3.1. Let $n$ be as given in (3.2a). If $b=1$ and $n$ is a bi-unitary triperfect number, then $n=120=2^{3} .3 .5$.

Proof. Let $b=1$ and $n$ be a bi-unitary triperfect number. Hence $n$ satisfies $(3.2 \mathrm{~b})(b=1)$. Taking $b=1$ in $(3.2 \mathrm{~b})$, we get $2^{3} \cdot v=6 \cdot \sigma^{* *}(v)$ or

$$
2^{2} \cdot v=3 . \sigma^{* *}(v)
$$

hence $3 \mid v$. Let $v=3^{c} . w$, where $(w, 2.3 .5)=1$. From $(3.2 \mathrm{a})(b=1)$, we get

$$
n=2^{3} \cdot 5 \cdot 3^{c} \cdot w
$$

and from (3.2d),

$$
2^{2} \cdot 3^{c-1} \cdot w=\sigma^{* *}\left(3^{c}\right) \cdot \sigma^{* *}(w),
$$

where $w$ can have at most one odd prime factor and $(w, 2.3 .5)=1$.

Let $c=1$. From (3.3b), we get $2^{2} . w=4 . \sigma^{* *}(w)$ or $w=\sigma^{* *}(w)$; hence $w=1$. Thus when $c=1,(3.3 \mathrm{~b})$ is satisfied. Hence $n=2^{3} .5 .3=120$ is a bi-unitary triperfect number.

Let $c=2$. From (3.3b) $(c=2)$, we get $2^{2} \cdot 3 \cdot w=10 \cdot \sigma^{* *}(w)$ and this implies that $5 \mid w$. This is false since $w$ is prime to 5 .

So we may assume that $c \geq 3$. By Lemma $2.1(\ell=2)$,

$$
\frac{\sigma^{* *}\left(3^{c}\right)}{3^{c}} \geq \frac{1}{3^{4}} \cdot\left(\frac{3^{5}-1}{2}-3^{2}\right)=\frac{112}{81} \quad(c \geq 3) .
$$

Hence from (3.3a), we have

$$
3=\frac{\sigma^{* *}(n)}{n} \geq \frac{15}{8} \cdot \frac{6}{5} \cdot \frac{112}{81}=3.11>3,
$$

a contradiction.

The proof of Lemma 3.1 is complete.

Lemma 3.2. Let $n$ be as in (3.2a). If $b \geq 2$ and $3 \mid n$, then $n$ cannot be a bi-unitary triperfect number.

Proof. Let $n$ be a bi-unitary triperfect number with $b \geq 2$. The relevant equations are (3.2a) and (3.2b). We assume that $3 \mid n$. Hence $v=3^{c} . w$, where $(w, 2.3 .5)=1$. In this case we have from (3.2a) and (3.2b),

$$
n=2^{3} .5^{b} .3^{c} . w
$$

and

$$
2^{3} \cdot 5^{b-1} \cdot 3^{c} \cdot w=\sigma^{* *}\left(5^{b}\right) \cdot \sigma^{* *}\left(3^{c}\right) \cdot \sigma^{* *}(w)
$$

where

$$
w \text { has no more than one odd prime factor. }
$$

Let $b=2$. Since $\sigma^{* *}\left(5^{2}\right)=26$, taking $b=2$ in (3.2b), after simplification we get

$$
2^{2} \cdot 5 \cdot 3^{c} \cdot w=13 \cdot \sigma^{* *}\left(3^{c}\right) \cdot \sigma^{* *}(w) .
$$


From (3.4d), 13|w. Let $w=13^{d}$. Hence from (3.4a),

$$
n=2^{3} \cdot 5^{2} \cdot 3^{c} \cdot 13^{d}
$$

and (3.4d) reduces to

$$
2^{2} \cdot 5 \cdot 3^{c} \cdot 13^{d-1}=\sigma^{* *}\left(3^{c}\right) \cdot \sigma^{* *}\left(13^{d}\right) .
$$

If $d=1$, (3.5b) $(d=1)$ implies that 7 divides the left-hand side of it. This is false. Similarly, taking $d=2$ in (3.5b), we see that 17 divides the left-hand side of it. Again this is false.

Hence we may assume that $d \geq 3$.

Taking $c=1$ in (3.5b), we find that $2^{3}$ divides the right-hand side of it while $2^{2}$ is a unitary divisor of its left-hand side. Also, taking $c=2$ in (3.5b), we obtain $2.3^{2} .13^{d-1}=\sigma^{* *}\left(13^{d}\right)$; this implies that $13 \mid \sigma^{* *}\left(13^{d}\right)$ which is false. Since $\sigma^{* *}\left(3^{3}\right)=\frac{3^{4}-1}{2}=40=2^{3} .5$ and $\sigma^{* *}\left(3^{4}\right)=\left(\frac{3^{2}-1}{2}\right) \cdot\left(3^{3}+1\right)=2^{4} .7$, it follows that $2^{3} \mid \sigma^{* *}\left(3^{c}\right)$ when $c=3$ or $c=4$. Hence if $c=3$ or $c=4$ in (3.5b), we find an imbalance between its two sides in powers of two. Hence we may assume that $c \geq 5$.

Since $c \geq 5$, by Lemma $2.1(\ell=3)$, we have

$$
\frac{\sigma^{* *}\left(3^{c}\right)}{3^{c}} \geq \frac{1}{3^{6}}\left(\frac{3^{7}-1}{2}-3^{3}\right)=\frac{1066}{729}
$$

and for $d \geq 3(\ell=2)$,

$$
\frac{\sigma^{* *}\left(13^{d}\right)}{13^{d}} \geq \frac{1}{13^{4}}\left(\frac{13^{5}-1}{12}-13^{2}\right)=\frac{30772}{28561}
$$

Since we can assume that $c \geq 5$ and $d \geq 3$, we have from (3.5a),

$$
3=\frac{\sigma^{* *}(n)}{n} \geq \frac{15}{8} \cdot \frac{26}{25} \cdot \frac{1066}{729} \cdot \frac{30772}{28561}=3.072179609>3
$$

a contradiction.

Thus $b=2$ cannot occur. We may assume that $b \geq 3$. The relevant equations are (3.4a) and (3.4b). Using the results $\frac{\sigma^{* *}\left(5^{b}\right)}{5^{b}} \geq \frac{756}{625}$ when $b \geq 3$, and $\frac{\sigma^{* *}\left(3^{c}\right)}{3^{c}} \geq \frac{112}{81},(c \geq 3)$ we have from (3.4a) for $c \geq 3$,

$$
3=\frac{\sigma^{* *}(n)}{n} \geq \frac{15}{8} \cdot \frac{756}{625} \cdot \frac{112}{81}=3.136>3,
$$

a contradiction. Hence $c=1$ or $c=2$.

Taking $c=1$ in (3.4b), we get $2.5^{b-1} .3 . w=\sigma^{* *}\left(5^{b}\right) \cdot \sigma^{* *}(w)$ which implies $w=1$ and hence it follows that $5 \mid \sigma^{* *}\left(5^{b}\right)$; but this is false. Hence $c=1$ is not possible.

Let $c=2$. Taking $c=2$ in (3.4a) and (3.4b), we obtain,

$$
n=2^{3} \cdot 5^{b} \cdot 3^{2} \cdot w, \quad(b \geq 3)
$$

and

$$
2^{2} \cdot 5^{b-2} \cdot 3^{2} \cdot w=\sigma^{* *}\left(5^{b}\right) \cdot \sigma^{* *}(w)
$$

where

$$
w \text { has not more than one odd prime factor. }
$$

We obtain a contradiction by examining the factors of $\sigma^{* *}\left(5^{b}\right)$. 
Case I. Let $b$ be odd. Then

$$
\sigma^{* *}\left(5^{b}\right)=\frac{5^{b+1}-1}{4}=\frac{\left(5^{t}-1\right)\left(5^{t}+1\right)}{4} . \quad\left(t=\frac{b+1}{2}\right)
$$

Since $b \geq 3$, we have $t \geq 2$.

(i) Let $t$ be even. Then $8 \mid 5^{t}-1$ and for any $t, 2 \mid 5^{t}+1$. Hence $4 \mid \frac{\left(5^{t}-1\right)\left(5^{t}+1\right)}{4}=\sigma^{* *}\left(5^{b}\right)$. From (3.6b), we must have $w=1$. With this, (3.6b) reduces to $2^{2} \cdot 5^{b-2} \cdot 3^{2}=\sigma^{* *}\left(5^{b}\right)$; this implies that $5 \mid \sigma^{* *}\left(5^{b}\right)$, which is false.

(ii) Let $t$ be odd. We prove that $\frac{5^{t}-1}{4}$ is odd, $>1$ and divisible by a prime $p \geq 29$.

(A) Clearly, $4 \| 5^{t}-1$. Hence $\frac{5^{t}-1}{4}$ is odd; it is $>1$, since $t \geq 2$. Also, since $t$ is odd, $\frac{5^{t}-1}{4}$ is not divisible by $3,7,13,17$ or 23 ; trivially not divisible by 5 .

(B) Suppose that $11 \mid 5^{t}-1$. This holds if and only if $5 \mid t$. This implies $5^{5}-1 \mid 5^{t}-1$. Also, $5^{5}-1=2^{2} .11 .71$. Hence 11 and 71 are factors of $\frac{5^{t}-1}{4} \mid \sigma^{* *}\left(5^{b}\right)$. From (3.6b) it follows that 11 and 71 divide $w$. This contradicts (3.6c). Thus $11 \nmid 5^{t}-1$.

(C) Suppose that $19 \mid 5^{t}-1$. This is if and only if $9 \mid t$. So, $5^{9}-1=2^{2} \cdot 19.31 .829 \mid 5^{t}-1$ and so $\frac{5^{t}-1}{4} \mid \sigma^{* *}\left(5^{b}\right)$ is divisible by three prime factors 19,31 and 829 . It follows form (3.6b) that these three prime factors divide $w$ contradicting $3.6 c$. Thus $19 \nmid 5^{t}-1$.

(D) From (A)-(C), we conclude that $\frac{5^{t}-1}{4}$ is not divisible by any prime in $[3,23]$; hence it must be divisible by a prime $p \geq 29$. Since $p \mid \sigma^{* *}\left(5^{b}\right)$, from(3.6b), $p \mid w$. From (3.6c), $w=p^{d}$. Substituting this into (3.6a), we obtain $n=2^{3} \cdot 5^{b} \cdot 3^{2} \cdot p^{d}$. Hence

$$
3=\frac{\sigma^{* *}(n)}{n}<\frac{15}{8} \cdot \frac{5}{4} \cdot \frac{10}{9} \cdot \frac{29}{28}=2.697172619<3
$$

a contradiction.

Case I is complete.

Case II. Let $b$ be even and $b=2 k$ say. Then

$$
\sigma^{* *}\left(5^{b}\right)=\left(\frac{5^{k}-1}{4}\right) \cdot\left(5^{k+1}+1\right) .
$$

(iii) Let $k$ be even. Then as in (i) of Case I, $4 \mid \sigma^{* *}\left(5^{b}\right)$. This leads to a contradiction from (3.6b) as before.

(iv) Let $k$ be odd. Since $b \geq 3$, we have $k \geq 3$; $\frac{5^{k}-1}{4}>1$, odd and must be divisible by a prime $p \geq 29$ and this gives a contradiction as in (ii)(D) of Case I.

Case II and thus the case $c=2$ is complete.

With this we have completed the proof of Lemma 3.2.

Lemma 3.3. Let $n$ be as in (3.2a). If $b \geq 2$ and $3 \nmid n$, then $n$ cannot be a bi-unitary triperfect number. 
Proof. Let $b \geq 2$ and $3 \nmid n$. Assume that $n$ is a bi-unitary triperfect number. We return to the equations (3.2a) and (3.2b). Again we consider $\sigma^{* *}\left(5^{b}\right)$ for obtaining a contradiction.

(i) If $b$ is odd or $4 \mid b$, then $3 \mid \sigma^{* *}\left(5^{b}\right)$. From (3.2b), it follows that $3 \mid v$ and so $3 \mid n$. But we have assumed that $3 \nmid n$.

(ii) Let $b=2 k$ and $k$ be odd. $b \geq 2$ implies that $k \geq 3 ; \frac{5^{k}-1}{4}>1$, odd and not divisible by $3,5,7,13,17$ and 23 as in (ii)(A) of Case I in Lemma 3.2.

(iii) Suppose $11 \mid 5^{k}-1$. This is if and only if $5 \mid k$ and this implies that $5^{5}-1=2^{2} .11 .71 \mid 5^{k}-1$. Hence 11 and 71 divide $\frac{5^{k}-1}{4} \mid \sigma^{* *}\left(5^{b}\right)$. From (3.2b), we infer that $v$ is divisible by 11 and 71 so that $v=11^{c} .71^{d}$. Hence from (3.2a), $n=2^{3} .5^{b} .11^{c} .71^{d}$ so that by Lemma 2.1,

$$
3=\frac{\sigma^{* *}(n)}{n}<\frac{15}{8} \cdot \frac{5}{4} \cdot \frac{11}{10} \cdot \frac{71}{70}=2.614955357<3,
$$

a contradiction. Hence $11 \nmid 5^{k}-1$.

(iv) That $19 \nmid 5^{k}-1$ can be proved exactly as in (ii)(C) of Case I in Lemma 3.2.

Thus $\frac{5^{k}-1}{4}$ is not divisible by any prime in $[3,23]$; being $>1$ and odd, it must be divisible by a prime $p \geq 29$. By (3.2b), $p \mid v$. Since $v$ has no more than two odd prime factors, let $q$ be the other possible odd prime factor of $v$; we may assume that $q \geq 7$. We can assume that $v=p^{c} . q^{d}$ and so from (3.2a), $n=2^{a} \cdot 5^{b} \cdot p^{c} \cdot q^{d}$. This implies

$$
3=\frac{\sigma^{* *}(n)}{n}<\frac{15}{8} \cdot \frac{5}{4} \cdot \frac{29}{28} \cdot \frac{7}{6}=2.83203125<3,
$$

a contradiction.

This completes the proof of Lemma 3.3.

Completion of proof of Theorem 3.1. Follows from Lemmas 3.1, 3.2 and 3.3.

\section{References}

[1] Cohen, E. (1960). Arithmetical functions associated with the unitary divisors of an integer, Math. Z., 74, 66-80.

[2] Dickson, L. E. (1919). History of the theory of numbers, Volume-I (Divisibility and Primality) AMS Chelsea Publishing, American Mathematical Society, Providence, Rhode Island, USA.

[3] Guy, R. K. (1981). Unsolved problems in number theory, Springer-Verlag.

[4] Harris, V. C. \& Subbarao, M. V. (1974). Unitary multiperfect numbers (Abstract), Notices Amer. Math. Soc., 21, A435. 
[5] Hagis, P., Jr. (1984). Lower bound for unitary multiperfect numbers, Fibonacci Quart., 22, $140-143$.

[6] Hagis, P., Jr. (1987). Bi-unitary amicable and multiperfect numbers, Fibonacci Quart., 25 (2), 144-150.

[7] Ochem, P. \& Rao, M. (2012). Odd perfect numbers are greater than $10^{1500}$, Math. Comp., 81, 1869-1877.

[8] Sándor, J. \& Crstici, P. (2004). Handbook of Number Theory II, Kluwer Academic.

[9] Sitaramaiah, V. \& Subbarao, M. V. (1998). On Unitary Multiperfect Numbers, Nieuw Arch. Wiskunde, 16, 57-61.

[10] Subbarao, M. V. (1970). Are there infinity of unitary perfect numbers? Amer. Math. Monthly, 77, 389-390.

[11] Subbarao, M. V. \& Warren, L. J. (1966). Unitary perfect numbers, Canad. Math. Bull. 9, 147-153.

[12] Suryanarayana, D. (1972). The number of bi-unitary divisors of an integer, in The Theory of Arithmetic Functions, Lecture Notes in Mathematics 251: 273-282, New York, SpringerVerlag.

[13] Vaithyanathaswamy, R. (1931). The theory of multiplicative arithmetic functions, Trans. Amer. Math. Soc., 33, 579-662.

[14] Wall, C. R. (1969). A new unitary perfect number, Notices Amer. Math. Soc., 16, 825.

[15] Wall, C. R. (1972). Bi-unitary perfect numbers, Proc. Amer. Math. Soc., 33, No. 1, 39-42.

[16] Wall, C. R. (1975). The fifth unitary perfect number, Canad. Math. Bull., 18, 115-122.

[17] Wikipedia contributors. (2019, September 28). Multiply perfect number. In Wikipedia, The Free Encyclopedia. Retrieved 2020, March 5, from https://en.wikipedia.org/ w/index .php?title=Multiply_perfect_number\&oldid=918383187 


\section{Appendix A Bi-unitary multiperfect numbers $<8.10^{10}$}

\begin{tabular}{|c|c|c|c|}
\hline SL.No & $k \geq 3$ & $n$ & Factorization \\
\hline 1 & 3 & 120 & $2^{3} \cdot 3 \cdot 5$ \\
2 & 3 & 672 & $2^{5} \cdot 3 \cdot 7$ \\
3 & 3 & 2160 & $2^{4} \cdot 3^{3} \cdot 5$ \\
4 & 3 & 10080 & $2^{5} \cdot 3^{2} \cdot 5 \cdot 7$ \\
5 & 3 & 22848 & $2^{6} \cdot 3 \cdot 7 \cdot 17$ \\
6 & 4 & 30240 & $2^{5} \cdot 3^{3} \cdot 5 \cdot 7$ \\
7 & 3 & 342720 & $2^{6} \cdot 3^{2} \cdot 5 \cdot 7 \cdot 17$ \\
8 & 3 & 523776 & $2^{9} \cdot 3 \cdot 11 \cdot 31$ \\
9 & 4 & 1028160 & $2^{6} \cdot 3^{3} \cdot 5 \cdot 7 \cdot 17$ \\
10 & 3 & 1528800 & $2^{5} \cdot 3 \cdot 5^{2} \cdot 7^{2} \cdot 13$ \\
11 & 4 & 6168960 & $2^{7} \cdot 3^{4} \cdot 5 \cdot 7 \cdot 17$ \\
12 & 3 & 7856640 & $2^{9} \cdot 3^{2} \cdot 5 \cdot 11 \cdot 31$ \\
13 & 4 & 7983360 & $2^{8} \cdot 3^{4} \cdot 5 \cdot 7 \cdot 11$ \\
14 & 3 & 14443520 & $2^{10} \cdot 5 \cdot 7 \cdot 13 \cdot 31$ \\
15 & 3 & 22932000 & $2^{5} \cdot 3^{2} \cdot 5^{3} \cdot 7^{2} \cdot 13$ \\
16 & 4 & 23569920 & $2^{9} \cdot 3^{3} \cdot 5 \cdot 11 \cdot 31$ \\
17 & 4 & 43330560 & $2^{10} \cdot 3 \cdot 5 \cdot 7 \cdot 13 \cdot 31$ \\
18 & 3 & 44553600 & $2^{7} \cdot 3^{2} \cdot 5^{2} \cdot 7 \cdot 13 \cdot 17$ \\
19 & 3 & 51979200 & $2^{6} \cdot 3 \cdot 5^{2} \cdot 7^{2} \cdot 13 \cdot 17$ \\
20 & 3 & 57657600 & $2^{8} \cdot 3^{2} \cdot 5^{2} \cdot 7 \cdot 11 \cdot 13$ \\
21 & 4 & 68796000 & $2^{5} \cdot 3^{3} \cdot 5^{3} \cdot 7^{2} \cdot 13$ \\
22 & 4 & 133660800 & $2^{7} \cdot 3^{3} \cdot 5^{2} \cdot 7 \cdot 13 \cdot 17$ \\
23 & 4 & 172972800 & $2^{8} \cdot 3^{3} \cdot 5^{2} \cdot 7 \cdot 11 \cdot 13$ \\
24 & 3 & 779688000 & $2^{6} \cdot 3^{2} \cdot 5^{3} \cdot 7^{2} \cdot 13 \cdot 17$ \\
25 & 3 & 1476304896 & $2^{13} \cdot 3 \cdot 11 \cdot 43 \cdot 127$ \\
26 & 4 & 2339064000 & $2^{6} \cdot 3^{3} \cdot 5^{3} \cdot 7^{2} \cdot 13 \cdot 17$ \\
27 & 3 & 6840038400 & $2^{10} \cdot 3 \cdot 5^{2} \cdot 13^{2} \cdot 17 \cdot 31$ \\
28 & 4 & 14034384000 & $2^{7} \cdot 3^{4} \cdot 5^{3} \cdot 7^{2} \cdot 13 \cdot 17$ \\
29 & 4 & 18162144000 & $2^{8} \cdot 3^{4} \cdot 5^{3} \cdot 7^{2} \cdot 11 \cdot 13$ \\
30 & 3 & 22144573440 & $2^{13} \cdot 3^{2} \cdot 5 \cdot 11 \cdot 43 \cdot 127$ \\
31 & 4 & 66433720320 & $2^{13} \cdot 3^{3} \cdot 5 \cdot 11 \cdot 43 \cdot 127$ \\
\hline
\end{tabular}

Note. SL.No 1 to 13 are due to Peter Hagis (Hagis, P., Jr. (1987). Bi-unitary amicable and multiperfect numbers, Fibonacci Quart., 25 (2), 144-150. ). 


\section{Appendix B $\quad \operatorname{ord}_{p} 5$}

Let $p$ be an odd prime $\neq 5$. In the following table, $r$ denotes the smallest positive integer such that $5^{r} \equiv 1(\bmod p)$; that is, $r=\operatorname{ord}_{p} 5$; $s$ denotes the smallest positive integer such that $5^{s} \equiv-1(\bmod p)$ if $s$ exists; if $s$ does not exist, that is, if $5^{t}+1$ is not divisible by $p$ for any positive integer $t$, the entry in column $s$ will be filled up by dash sign. If $r$ is even, then $s=r / 2$, and if $r$ is odd, $s$ does not exist.

Table-I

\begin{tabular}{|c|c|c|c||c|c|c|c||c|c|c|c|}
\hline SL.No & $\mathrm{p}$ & $\mathrm{r}$ & $\mathrm{s}$ & SL.No & $\mathrm{p}$ & $\mathrm{r}$ & $\mathrm{s}$ & SL.No & $\mathrm{p}$ & $\mathrm{r}$ & $\mathrm{s}$ \\
\hline 1 & 3 & 2 & 1 & 11 & 37 & 36 & 18 & 21 & 79 & 39 & - \\
2 & 5 & - & - & 12 & 41 & 20 & 10 & 22 & 83 & 82 & 41 \\
3 & 7 & 6 & 3 & 13 & 43 & 42 & 21 & 23 & 89 & 44 & 22 \\
4 & 11 & 5 & - & 14 & 47 & 46 & 23 & 24 & 97 & 96 & 48 \\
5 & 13 & 4 & 2 & 15 & 53 & 52 & 26 & 25 & 101 & 25 & - \\
6 & 17 & 16 & 8 & 16 & 59 & 29 & - & 26 & 103 & 102 & 51 \\
7 & 19 & 9 & - & 17 & 61 & 30 & 15 & 27 & 107 & 106 & 53 \\
8 & 23 & 22 & 11 & 18 & 67 & 22 & 11 & 28 & 109 & 27 & - \\
9 & 29 & 14 & 7 & 19 & 71 & 5 & - & 29 & 113 & 112 & 56 \\
10 & 31 & 3 & - & 20 & 73 & 72 & 36 & 30 & 127 & 42 & 21 \\
\hline
\end{tabular}

Table-II

\begin{tabular}{|c|c|c|c||c|c|c|c||c|c|c|c|}
\hline SL.No & $\mathrm{p}$ & $\mathrm{r}$ & $\mathrm{s}$ & SL.No & $\mathrm{p}$ & $\mathrm{r}$ & $\mathrm{s}$ & SL.No & $\mathrm{p}$ & $\mathrm{r}$ & $\mathrm{s}$ \\
\hline 31 & 131 & 65 & - & 57 & 271 & 27 & - & 83 & 433 & 432 & 216 \\
32 & 137 & 136 & 68 & 58 & 277 & 276 & 138 & 84 & 439 & 219 & - \\
33 & 139 & 69 & - & 59 & 281 & 140 & 70 & 85 & 443 & 442 & 221 \\
34 & 149 & 37 & - & 60 & 283 & 282 & 141 & 86 & 449 & 14 & 7 \\
35 & 151 & 75 & - & 61 & 293 & 292 & 146 & 87 & 457 & 152 & 76 \\
36 & 157 & 156 & 78 & 62 & 307 & 306 & 153 & 88 & 461 & 115 & - \\
37 & 163 & 54 & 27 & 63 & 311 & 155 & - & 89 & 463 & 462 & 231 \\
38 & 167 & 166 & 83 & 64 & 313 & 8 & 4 & 90 & 467 & 466 & 233 \\
39 & 173 & 172 & 86 & 65 & 317 & 316 & 158 & 91 & 479 & 239 & - \\
40 & 179 & 89 & - & 66 & 331 & 165 & - & 92 & 487 & 54 & 27 \\
41 & 181 & 15 & - & 67 & 337 & 112 & 56 & 93 & 491 & 245 & - \\
42 & 191 & 19 & - & 68 & 347 & 346 & 173 & 94 & 499 & 249 & - \\
43 & 193 & 192 & 96 & 69 & 349 & 174 & 87 & 95 & 503 & 502 & - \\
44 & 197 & 196 & 98 & 70 & 353 & 352 & 176 & 96 & 509 & 254 & - \\
45 & 199 & 33 & - & 71 & 359 & 179 & - & 97 & 521 & 10 & 5 \\
46 & 211 & 35 & - & 72 & 367 & 122 & 61 & 98 & 523 & 522 & 261 \\
47 & 223 & 222 & 111 & 73 & 373 & 372 & 186 & 99 & 541 & 135 & - \\
48 & 227 & 226 & 113 & 74 & 379 & 21 & - & 100 & 547 & 546 & 273 \\
49 & 229 & 114 & 57 & 75 & 383 & 382 & 191 & 101 & 557 & 556 & 278 \\
50 & 233 & 232 & 116 & 76 & 389 & 97 & - & 102 & 563 & 562 & - \\
51 & 239 & 119 & - & 77 & 397 & 396 & 198 & 103 & 569 & 71 & - \\
52 & 241 & 40 & 20 & 78 & 401 & 25 & - & 104 & 571 & 285 & - \\
53 & 251 & 25 & - & 79 & 409 & 17 & - & 105 & 577 & 576 & 288 \\
54 & 257 & 256 & 128 & 80 & 419 & 209 & - & 106 & 587 & 586 & 293 \\
55 & 263 & 262 & - & 81 & 421 & 210 & 105 & & & & \\
56 & 269 & 67 & - & 82 & 431 & 215 & - & & & & \\
\hline
\end{tabular}


Table-III

\begin{tabular}{|c|c|c|c||c|c|c|c||c|c|c|c|}
\hline SL.No & $p$ & $r$ & $s$ & SL.No & $\mathrm{p}$ & $\mathrm{r}$ & $\mathrm{s}$ & SL.No & $\mathrm{p}$ & $\mathrm{r}$ & $\mathrm{s}$ \\
\hline 107 & 593 & 592 & 296 & 132 & 751 & 375 & - & 157 & 929 & 232 & 116 \\
108 & 599 & 299 & - & 133 & 757 & 756 & 378 & 158 & 937 & 936 & 468 \\
109 & 601 & 12 & 6 & 134 & 761 & 38 & 19 & 159 & 941 & 235 & - \\
110 & 607 & 606 & 303 & 135 & 769 & 128 & 64 & 160 & 947 & 946 & 473 \\
111 & 613 & 612 & 306 & 136 & 773 & 772 & 386 & 161 & 953 & 952 & 476 \\
112 & 617 & 616 & 308 & 137 & 787 & 786 & 393 & 162 & 967 & 966 & 483 \\
113 & 619 & 309 & - & 138 & 797 & 796 & 398 & 163 & 971 & 485 & - \\
114 & 631 & 35 & - & 139 & 809 & 404 & 202 & 164 & 977 & 976 & 488 \\
115 & 641 & 64 & 32 & 140 & 811 & 405 & - & 165 & 983 & 982 & 491 \\
116 & 643 & 214 & 107 & 141 & 821 & 410 & 205 & 166 & 991 & 495 & - \\
117 & 647 & 646 & 323 & 142 & 823 & 274 & 137 & 167 & 997 & 332 & 166 \\
118 & 653 & 652 & 326 & 143 & 827 & 118 & 59 & 168 & 1009 & 504 & 252 \\
119 & 659 & 329 & - & 144 & 829 & 9 & - & 169 & 1013 & 1012 & 506 \\
120 & 661 & 330 & 165 & 145 & 839 & 419 & - & 170 & 1019 & 509 & - \\
121 & 673 & 672 & 336 & 146 & 853 & 284 & 142 & 171 & 1021 & 255 & - \\
122 & 677 & 676 & 338 & 147 & 857 & 856 & 428 & 172 & 1031 & 515 & - \\
123 & 683 & 682 & 341 & 148 & 859 & 429 & - & 173 & 1033 & 1032 & 516 \\
124 & 691 & 115 & - & 149 & 863 & 862 & 431 & 174 & 1039 & 173 & - \\
125 & 701 & 350 & 175 & 150 & 877 & 876 & 438 & 175 & 1049 & 524 & 262 \\
126 & 709 & 354 & 177 & 151 & 881 & 440 & 220 & 176 & 1051 & 525 & - \\
127 & 719 & 359 & - & 152 & 883 & 126 & 73 & 177 & 1061 & 265 & - \\
128 & 727 & 726 & 363 & 153 & 887 & 886 & 443 & 178 & 1063 & 354 & 177 \\
129 & 733 & 244 & 122 & 154 & 907 & 906 & 453 & 179 & 1069 & 267 & - \\
130 & 739 & 123 & - & 155 & 911 & 455 & & 180 & 1087 & 362 & 181 \\
131 & 743 & 742 & 371 & 156 & 919 & 459 & - & 181 & 1091 & 545 & - \\
\hline
\end{tabular}


Table-IV

\begin{tabular}{|c|c|c|c||c|c|c|c||c|c|c|c|}
\hline SL.No & $\mathrm{p}$ & $\mathrm{r}$ & $\mathrm{s}$ & SL.No & $\mathrm{p}$ & $\mathrm{r}$ & $\mathrm{s}$ & SL.No & $\mathrm{p}$ & $\mathrm{r}$ & $\mathrm{s}$ \\
\hline 182 & 1093 & 1092 & 546 & 207 & 1283 & 1282 & 641 & 232 & 1471 & 735 & - \\
183 & 1097 & 1096 & 548 & 208 & 1289 & 644 & 322 & 233 & 1481 & 740 & 370 \\
184 & 1103 & 1102 & 551 & 209 & 1291 & 215 & - & 234 & 1483 & 1482 & $741-$ \\
185 & 1109 & 554 & 277 & 210 & 1297 & 144 & 62 & 235 & 1487 & 1486 & 743 \\
186 & 1117 & 372 & 186 & 211 & 1301 & 650 & 325 & 236 & 1489 & 372 & 186 \\
187 & 1123 & 374 & 187 & 212 & 1303 & 62 & 31 & 237 & 1493 & 1492 & 746 \\
188 & 1129 & 282 & 141 & 213 & 1307 & 1306 & 653 & 238 & 1499 & 749 & - \\
189 & 1151 & 575 & - & 214 & 1319 & 659 & - & 239 & 1511 & 755 & - \\
190 & 1153 & 1152 & 576 & 215 & 1321 & 660 & 330 & 240 & 1523 & 1522 & 761 \\
191 & 1163 & 1162 & 581 & 216 & 1327 & 340 & 170 & 241 & 1531 & 85 & - \\
192 & 1171 & 45 & - & 217 & 1361 & 1360 & 680 & 242 & 1543 & 1542 & 771 \\
193 & 1181 & 590 & 295 & 218 & 1367 & 1366 & 683 & 243 & 1549 & 86 & 43 \\
194 & 1187 & 1186 & 593 & 219 & 1373 & 1372 & 686 & 244 & 1553 & 1552 & 776 \\
195 & 1193 & 1192 & 596 & 220 & 1381 & 690 & 345 & 245 & 1559 & 779 & - \\
196 & 1201 & 600 & 300 & 221 & 1399 & 699 & - & 246 & 1567 & 1566 & 783 \\
197 & 1213 & 1212 & 606 & 222 & 1409 & 44 & 22 & 247 & 1571 & 785 & - \\
198 & 1217 & 1216 & 608 & 223 & 1423 & 704 & 352 & 248 & 1579 & 789 & - \\
199 & 1223 & 1222 & 611 & 224 & 1427 & 1426 & 713 & 249 & 1583 & 1582 & 791 \\
200 & 1229 & 614 & 307 & 225 & 1429 & 119 & - & 250 & 1597 & 532 & 266 \\
201 & 1231 & 615 & - & 226 & 1433 & 1432 & 716 & 251 & 1601 & 400 & 200 \\
202 & 1237 & 1236 & 618 & 227 & 1439 & 719 & - & 252 & 1607 & 1606 & 803 \\
203 & 1249 & 624 & 312 & 228 & 1447 & 482 & 241 & 253 & 1609 & 67 & - \\
204 & 1259 & 629 & - & 229 & 1451 & 725 & - & 254 & 1613 & 1612 & 806 \\
205 & 1277 & 1276 & 638 & 230 & 1453 & 132 & 61 & 255 & 1619 & 809 & - \\
206 & 1279 & 639 & - & 231 & 1459 & 243 & - & 256 & 1621 & 405 & - \\
\hline
\end{tabular}


Table-V

\begin{tabular}{|c|c|c|c||c|c|c|c||c|c|c|c|}
\hline SL.No & $\mathrm{p}$ & $\mathrm{r}$ & $\mathrm{s}$ & SL.No & $\mathrm{p}$ & $\mathrm{r}$ & $\mathrm{s}$ & SL.No & $\mathrm{p}$ & $\mathrm{r}$ & $\mathrm{s}$ \\
\hline 257 & 1627 & 542 & 271 & 282 & 1847 & 1846 & 923 & 307 & 2029 & 1014 & 507 \\
258 & 1637 & 1636 & 818 & 283 & 1861 & 31 & - & 308 & 2039 & 1019 & - \\
259 & 1657 & 552 & 276 & 284 & 1867 & 622 & 311 & 309 & 2053 & 2052 & 1026 \\
260 & 1663 & 1662 & 831 & 285 & 1871 & 935 & - & 310 & 2063 & 2062 & 1031 \\
261 & 1667 & 1666 & 833 & 286 & 1873 & 624 & 312 & 311 & 2069 & 94 & 37 \\
262 & 1669 & 834 & 417 & 287 & 1877 & 1876 & 938 & 312 & 2081 & 520 & 260 \\
263 & 1693 & 1692 & 846 & 288 & 1879 & 939 & - & 313 & 2083 & 2082 & 1041 \\
264 & 1697 & 1696 & 848 & 289 & 1889 & 472 & 236 & 314 & 2087 & 2086 & 1043 \\
265 & 1699 & 283 & - & 290 & 1901 & 50 & 25 & 315 & 2089 & 1044 & 522 \\
266 & 1709 & 854 & 427 & 291 & 1907 & 1906 & 953 & 316 & 2099 & 1049 & - \\
267 & 1721 & 430 & 860 & 292 & 1913 & 965 & - & 317 & 2111 & 1055 & - \\
268 & 1723 & 574 & 287 & 293 & 1931 & 965 & - & 318 & 2113 & 2112 & 1056 \\
269 & 1733 & 1732 & 866 & 294 & 1933 & 1932 & 966 & 319 & 2129 & 266 & 133 \\
270 & 1741 & 15 & - & 295 & 1949 & 487 & - & 320 & 2131 & 1065 & - \\
271 & 1747 & 1746 & 873 & 296 & 1951 & 975 & - & 321 & 2137 & 712 & 356 \\
272 & 1753 & 584 & 292 & 297 & 1973 & 68 & 34 & 322 & 2141 & 1070 & 535 \\
273 & 1759 & 293 & - & 298 & 1979 & 989 & - & 323 & 2143 & 2142 & 1071 \\
274 & 1777 & 1776 & 888 & 299 & 1987 & 1986 & 993 & 324 & 2153 & 2152 & 1076 \\
275 & 1783 & 162 & 81 & 300 & 1993 & 1992 & 996 & 325 & 2161 & 1080 & 540 \\
276 & 1787 & 1786 & 893 & 301 & 1997 & 1996 & 998 & 326 & 2179 & 1089 & - \\
277 & 1789 & 894 & 447 & 302 & 1999 & 999 & - & 327 & 2203 & 2202 & 1101 \\
278 & 1801 & 900 & 450 & 303 & 2003 & 2002 & 1001 & 328 & 2207 & 2206 & 1103 \\
279 & 1811 & 905 & - & 304 & 2011 & 1005 & - & 329 & 2213 & 2212 & 1106 \\
280 & 1823 & 1822 & 911 & 305 & 2017 & 2016 & 1008 & 330 & 2221 & 370 & 185 \\
281 & 1831 & 305 & - & 306 & 2027 & 2026 & 1013 & 331 & 2237 & 2236 & 1118 \\
\hline
\end{tabular}

Table-VI

\begin{tabular}{|c|c|c|c||c|c|c|c|}
\hline SL.No & $\mathrm{p}$ & $\mathrm{r}$ & $\mathrm{s}$ & SL.No & $\mathrm{p}$ & $\mathrm{r}$ & $\mathrm{s}$ \\
\hline 332 & 2239 & 373 & - & 351 & 2377 & 2376 & 1188 \\
333 & 2243 & 2242 & 1121 & 352 & 2381 & 238 & 119 \\
334 & 2251 & 1125 & - & 353 & 2383 & 2382 & 1191 \\
335 & 2267 & 2266 & 1133 & 354 & 2389 & 398 & 194 \\
336 & 2269 & 567 & - & 355 & 2393 & 2392 & 1196 \\
337 & 2273 & 2272 & 1136 & 356 & 2399 & 1199 & - \\
338 & 2281 & 60 & 30 & 357 & 2411 & 1205 & - \\
339 & 2287 & 254 & 127 & 358 & 2417 & 2416 & 1208 \\
340 & 2293 & 2292 & 1146 & 359 & 2423 & 2422 & 1211 \\
341 & 2297 & 2296 & 1148 & 360 & 2437 & 2436 & 1218 \\
342 & 2309 & 577 & - & 361 & 2441 & 305 & - \\
343 & 2311 & 165 & - & 362 & 2447 & 2446 & 1223 \\
344 & 2333 & 1169 & - & 363 & 2459 & 1229 & - \\
345 & 2339 & 2338 & 1169 & 364 & 2467 & 2466 & 1233 \\
346 & 2341 & 1170 & 585 & 365 & 2473 & 2472 & 1236 \\
347 & 2347 & 2346 & 1173 & 366 & 2477 & 2476 & 1238 \\
348 & 2351 & 1175 & - & 367 & 2503 & 2502 & 1251 \\
349 & 2357 & 2356 & 1178 & & & & \\
350 & 2371 & 1185 & - & & & & \\
\hline
\end{tabular}




\section{Appendix C $\quad \operatorname{ord}_{p} 7$}

Let $p$ be an odd prime $\neq 7$. In the following table, $r$ denotes the smallest positive integer such that $7^{r} \equiv 1(\bmod p)$; that is, $r=\operatorname{ord}_{p} 7 ; s$ denotes the smallest positive integer such that $7^{s} \equiv-1(\bmod p)$ if $s$ exists; if $s$ does not exist, that is, if $7^{t}+1$ is not divisible by $p$ for any positive integer $t$, the entry in column $s$ will be filled up by dash sign. If $r$ is even, then $s=r / 2$, and if $r$ is odd, $s$ does not exist.

Table-I

\begin{tabular}{|c|c|c|c||c|c|c|c||c|c|c|c|}
\hline SL.No & $p$ & $r$ & $s$ & SL.No & $p$ & $r$ & $s$ & SL.No & $p$ & $r$ & $s$ \\
\hline 1 & 3 & 1 & - & 11 & 37 & 9 & - & 21 & 79 & 78 & 39 \\
2 & 5 & 4 & 2 & 12 & 41 & 40 & 20 & 22 & 83 & 41 & - \\
3 & 7 & - & - & 13 & 43 & 6 & 3 & 23 & 89 & 88 & 44 \\
4 & 11 & 10 & 5 & 14 & 47 & 23 & - & 24 & 97 & 96 & 48 \\
5 & 13 & 12 & 6 & 15 & 53 & 26 & 13 & 25 & 101 & 100 & 50 \\
6 & 17 & 16 & 8 & 16 & 59 & 29 & - & 26 & 103 & 51 & - \\
7 & 19 & 3 & - & 17 & 61 & 60 & 30 & 27 & 107 & 106 & 53 \\
8 & 23 & 22 & 11 & 18 & 67 & 66 & 33 & 28 & 109 & 27 & - \\
9 & 29 & 7 & - & 19 & 71 & 70 & 35 & 29 & 113 & 14 & 7 \\
10 & 31 & 15 & - & 20 & 73 & 24 & 12 & 30 & 127 & 126 & 63 \\
\hline
\end{tabular}

Table-II

\begin{tabular}{|c|c|c|c||c|c|c|c||c|c|c|c|}
\hline SL.No & $p$ & $r$ & $s$ & SL.No & $p$ & $r$ & $s$ & SL.No & $p$ & $r$ & $s$ \\
\hline 31 & 131 & 65 & - & 57 & 271 & 135 & - & 83 & 433 & 432 & 216 \\
32 & 137 & 68 & 34 & 58 & 277 & 138 & 69 & 84 & 439 & 73 & - \\
33 & 139 & 69 & - & 59 & 281 & 20 & 10 & 85 & 443 & 442 & 221 \\
34 & 149 & 74 & 37 & 60 & 283 & 141 & - & 86 & 449 & 112 & 56 \\
35 & 151 & 150 & 75 & 61 & 293 & 292 & 146 & 87 & 457 & 114 & 57 \\
36 & 157 & 52 & 26 & 62 & 307 & 153 & - & 88 & 461 & 460 & 230 \\
37 & 163 & 162 & 81 & 63 & 311 & 31 & - & 89 & 463 & 154 & 77 \\
38 & 167 & 83 & - & 64 & 313 & 104 & 52 & 90 & 467 & 233 & - \\
39 & 173 & 172 & 86 & 65 & 317 & 158 & 79 & 91 & 479 & 239 & - \\
40 & 179 & 178 & 89 & 66 & 331 & 110 & 55 & 92 & 487 & 162 & 81 \\
41 & 181 & 12 & 6 & 67 & 337 & 56 & 28 & 93 & 491 & 490 & 245 \\
42 & 191 & 10 & 5 & 68 & 347 & 346 & 173 & 94 & 499 & 498 & 249 \\
43 & 193 & 24 & 12 & 69 & 349 & 348 & 174 & 95 & 503 & 251 & - \\
44 & 197 & 98 & 49 & 70 & 353 & 32 & 16 & 96 & 509 & 508 & 254 \\
45 & 199 & 99 & - & 71 & 359 & 358 & 179 & 97 & 521 & 520 & 260 \\
46 & 211 & 210 & 105 & 72 & 367 & 61 & - & 98 & 523 & 261 & - \\
47 & 223 & 37 & - & 73 & 373 & 62 & 31 & 99 & 541 & 90 & 45 \\
48 & 227 & 113 & - & 74 & 379 & 378 & 189 & 100 & 547 & 546 & 273 \\
49 & 229 & 228 & 114 & 75 & 383 & 191 & - & 101 & 557 & 278 & 139 \\
50 & 233 & 116 & 58 & 76 & 389 & 97 & - & 102 & 563 & 281 & - \\
51 & 239 & 238 & 119 & 77 & 397 & 396 & 198 & 103 & 569 & 284 & 142 \\
52 & 241 & 240 & 120 & 78 & 401 & 200 & 100 & 104 & 571 & 190 & 95 \\
53 & 251 & 125 & - & 79 & 409 & 24 & 12 & 105 & 577 & 576 & 288 \\
54 & 257 & 256 & 128 & 80 & 419 & 19 & - & 106 & 587 & 293 & - \\
55 & 263 & 262 & 131 & 81 & 421 & 70 & 35 & & & & \\
56 & 269 & 268 & 134 & 82 & 431 & 430 & 215 & & & & \\
\hline
\end{tabular}


Table-III

\begin{tabular}{|c|c|c|c||c|c|c|c||c|c|c|c|}
\hline SL.No & $p$ & $r$ & $s$ & SL.No & $p$ & $r$ & $s$ & SL.No & $p$ & $r$ & $s$ \\
\hline 107 & 593 & 592 & 296 & 132 & 751 & 250 & 125 & 157 & 929 & 928 & 464 \\
108 & 599 & 598 & 299 & 133 & 757 & 189 & - & 158 & 937 & 936 & 468 \\
109 & 601 & 600 & 300 & 134 & 761 & 760 & 380 & 159 & 941 & 940 & 470 \\
110 & 607 & 101 & - & 135 & 769 & 256 & 128 & 160 & 947 & 86 & 43 \\
111 & 613 & 153 & - & 136 & 773 & 772 & 386 & 161 & 953 & 238 & 119 \\
112 & 617 & 308 & 154 & 137 & 787 & 393 & - & 162 & 967 & 966 & 483 \\
113 & 619 & 309 & - & 138 & 797 & 796 & 398 & 163 & 971 & 97 & - \\
114 & 631 & 630 & 315 & 139 & 809 & 101 & - & 164 & 977 & 488 & 244 \\
115 & 641 & 320 & 160 & 140 & 811 & 27 & - & 165 & 983 & 491 & - \\
116 & 643 & 321 & - & 141 & 821 & 410 & 205 & 166 & 991 & 990 & 495 \\
117 & 647 & 323 & - & 142 & 823 & 822 & 411 & 167 & 997 & 996 & 498 \\
118 & 653 & 163 & - & 143 & 827 & 826 & 413 & 168 & 1009 & 252 & 126 \\
119 & 659 & 658 & 329 & 144 & 829 & 276 & 138 & 169 & 1013 & 1012 & 506 \\
120 & 661 & 44 & 22 & 145 & 839 & 419 & - & 170 & 1019 & 1018 & 509 \\
121 & 673 & 112 & 56 & 146 & 853 & 284 & 142 & 171 & 1021 & 340 & 170 \\
122 & 677 & 676 & 338 & 147 & 857 & 856 & 428 & 172 & 1031 & 206 & 103 \\
123 & 683 & 682 & 341 & 148 & 859 & 429 & - & 173 & 1033 & 172 & 86 \\
124 & 691 & 345 & - & 149 & 863 & 862 & 431 & 174 & 1039 & 519 & - \\
125 & 701 & 175 & - & 150 & 877 & 219 & - & 175 & 1049 & 1048 & 524 \\
126 & 709 & 177 & - & 151 & 881 & 80 & 40 & 176 & 1051 & 1050 & 525 \\
127 & 719 & 359 & - & 152 & 883 & 98 & 49 & 177 & 1061 & 265 & - \\
128 & 727 & 363 & - & 153 & 887 & 443 & - & 178 & 1063 & 9 & - \\
129 & 733 & 732 & 366 & 154 & 907 & 906 & 453 & 179 & 1069 & 1068 & 534 \\
130 & 739 & 738 & 369 & 155 & 911 & 14 & 7 & 180 & 1087 & 362 & 181 \\
131 & 743 & 742 & 371 & 156 & 919 & 918 & 459 & 181 & 1091 & 545 & - \\
\hline
\end{tabular}


Table-IV

\begin{tabular}{|c|c|c|c||c|c|c|c||c|c|c|c|}
\hline SL.No & $p$ & $r$ & $s$ & SL.No & $p$ & $r$ & $s$ & SL.No & $p$ & $r$ & $s$ \\
\hline 182 & 1093 & 273 & - & 207 & 1283 & 1282 & 641 & 232 & 1471 & 1470 & 735 \\
183 & 1097 & 1096 & 548 & 208 & 1289 & 322 & 161 & 233 & 1481 & 185 & - \\
184 & 1103 & 1102 & 551 & 209 & 1291 & 645 & - & 234 & 1483 & 741 & - \\
185 & 1109 & 1108 & 554 & 210 & 1297 & 648 & 324 & 235 & 1487 & 743 & - \\
186 & 1117 & 558 & 279 & 211 & 1301 & 1300 & 650 & 236 & 1489 & 496 & 248 \\
187 & 1123 & 11 & - & 212 & 1303 & 1302 & 651 & 237 & 1493 & 746 & 373 \\
188 & 1129 & 564 & 282 & 213 & 1307 & 653 & - & 238 & 1499 & 214 & 107 \\
189 & 1151 & 115 & - & 214 & 1319 & 659 & - & 239 & 1511 & 755 & - \\
190 & 1153 & 384 & 192 & 215 & 1321 & 264 & 132 & 240 & 1523 & 1522 & 761 \\
191 & 1163 & 1162 & 581 & 216 & 1327 & 442 & 221 & 241 & 1531 & 85 & - \\
192 & 1171 & 234 & 117 & 217 & 1361 & 1360 & 680 & 242 & 1543 & 257 & - \\
193 & 1181 & 1180 & 590 & 218 & 1367 & 1366 & 683 & 243 & 1549 & 774 & 387 \\
194 & 1187 & 1186 & 593 & 219 & 1373 & 343 & - & 244 & 1553 & 1552 & 776 \\
195 & 1193 & 1192 & 596 & 220 & 1381 & 345 & - & 245 & 1559 & 779 & - \\
196 & 1201 & 8 & 4 & 221 & 1399 & 699 & - & 246 & 1567 & 783 & - \\
197 & 1213 & 303 & - & 222 & 1409 & 44 & 22 & 247 & 1571 & 785 & - \\
198 & 1217 & 1216 & 608 & 223 & 1423 & 474 & 237 & 248 & 1579 & 526 & 263 \\
199 & 1223 & 611 & - & 224 & 1427 & 713 & - & 249 & 1583 & 226 & 113 \\
200 & 1229 & 614 & 307 & 225 & 1429 & 357 & - & 250 & 1597 & 798 & 399 \\
201 & 1231 & 615 & - & 226 & 1433 & 1432 & 716 & 251 & 1601 & 1600 & 800 \\
202 & 1237 & 1236 & 618 & 227 & 1439 & 1438 & 719 & 252 & 1607 & 1606 & 803 \\
203 & 1249 & 1248 & 624 & 228 & 1447 & 241 & - & 253 & 1609 & 1608 & 804 \\
204 & 1259 & 629 & - & 229 & 1451 & 1450 & 725 & 254 & 1613 & 1612 & 806 \\
205 & 1277 & 1276 & 638 & 230 & 1453 & 121 & - & 255 & 1619 & 1618 & 809 \\
206 & 1279 & 639 & - & 231 & 1459 & 243 & - & 256 & 1621 & 81 & - \\
\hline
\end{tabular}


Table-V

\begin{tabular}{|c|c|c|c||c|c|c|c||c|c|c|c|}
\hline SL.No & $p$ & $r$ & $s$ SL.No & $p$ & $r$ & $s$ & SL.No & $p$ & $r$ & $s$ & \\
\hline 257 & 1627 & 813 & - & 282 & 1847 & 923 & - & 307 & 2029 & 676 & 338 \\
258 & 1637 & 1636 & 818 & 283 & 1861 & 372 & 186 & 308 & 2039 & 2038 & 1019 \\
259 & 1657 & 184 & 92 & 284 & 1867 & 933 & - & 309 & 2053 & 342 & 171 \\
260 & 1663 & 554 & 277 & 285 & 1871 & 374 & 187 & 310 & 2063 & 1031 & - \\
261 & 1667 & 1666 & 833 & 286 & 1873 & 117 & - & 311 & 2069 & 517 & - \\
262 & 1669 & 1668 & 834 & 287 & 1877 & 938 & 469 & 312 & 2081 & 1040 & 520 \\
263 & 1693 & 1692 & 846 & 288 & 1879 & 939 & - & 313 & 2083 & 2082 & 1041 \\
264 & 1697 & 1696 & 848 & 289 & 1889 & 1888 & 944 & 314 & 2087 & 2086 & 1043 \\
265 & 1699 & 849 & - & 290 & 1901 & 950 & 475 & 315 & 2089 & 2088 & 1044 \\
266 & 1709 & 854 & 427 & 291 & 1907 & 953 & - & 316 & 2099 & 1049 & - \\
267 & 1721 & 1720 & 860 & 292 & 1913 & 956 & 478 & 317 & 2111 & 2110 & 1055 \\
268 & 1723 & 574 & 287 & 293 & 1931 & 965 & - & 318 & 2113 & 192 & 96 \\
269 & 1733 & 433 & - & 294 & 1933 & 966 & 483 & 319 & 2129 & 56 & 28 \\
270 & 1741 & 580 & 290 & 295 & 1949 & 1948 & 974 & 320 & 2131 & 355 & - \\
271 & 1747 & 1746 & 873 & 296 & 1951 & 975 & - & 321 & 2137 & 356 & 178 \\
272 & 1753 & 1752 & 876 & 297 & 1973 & 1972 & 986 & 322 & 2141 & 2140 & 1070 \\
273 & 1759 & 1758 & 879 & 298 & 1979 & 989 & - & 323 & 2143 & 2142 & 1071 \\
274 & 1777 & 592 & 296 & 299 & 1987 & 993 & - & 324 & 2153 & 269 & - \\
275 & 1783 & 891 & - & 300 & 1993 & 1992 & 996 & 325 & 2161 & 432 & 216 \\
276 & 1787 & 1786 & 893 & 301 & 1997 & 499 & - & 326 & 2179 & 2178 & 1089 \\
277 & 1789 & 894 & 447 & 302 & 1999 & 666 & 333 & 327 & 2203 & 1101 & \\
278 & 1801 & 450 & 225 & 303 & 2003 & 2002 & 1001 & 328 & 2207 & 2206 & 1103 \\
279 & 1811 & 181 & - & 304 & 2011 & 2010 & 1005 & 329 & 2213 & 553 & - \\
280 & 1823 & 911 & - & 305 & 2017 & 1008 & 504 & 330 & 2221 & 1110 & 555 \\
281 & 1831 & 1830 & 915 & 306 & 2027 & 2026 & 1013 & 331 & 2237 & 559 & - \\
\hline
\end{tabular}

Table-VI

\begin{tabular}{|c|c|c|c||c|c|c|c|}
\hline SL.No & $p$ & $r$ & $s$ & SL.No & $p$ & $r$ & $s$ \\
\hline 332 & 2239 & 373 & - & 351 & 2377 & 27 & - \\
333 & 2243 & 1121 & - & 352 & 2381 & 238 & 119 \\
334 & 2251 & 2250 & 1125 & 353 & 2383 & 397 & - \\
335 & 2267 & 1133 & - & 354 & 2389 & 194 & 97 \\
336 & 2269 & 567 & - & 355 & 2393 & 2392 & 1196 \\
337 & 2273 & 2272 & 1136 & 356 & 2399 & 1199 & - \\
338 & 2281 & 2280 & 1140 & 357 & 2411 & 1205 & - \\
339 & 2287 & 1143 & - & 358 & 2417 & 604 & 302 \\
340 & 2293 & 1146 & 573 & 359 & 2423 & 346 & 173 \\
341 & 2297 & 1148 & 574 & 360 & 2437 & 1218 & 609 \\
342 & 2309 & 2308 & 1154 & 361 & 2441 & 488 & 244 \\
343 & 2311 & 110 & 55 & 362 & 2447 & 2446 & 1223 \\
344 & 2333 & 583 & - & 363 & 2459 & 2458 & 1229 \\
345 & 2339 & 2338 & 1169 & 364 & 2467 & 1233 & - \\
346 & 2341 & 2340 & 1170 & 365 & 2473 & 1236 & 618 \\
347 & 2347 & 782 & 391 & 366 & 2477 & 2476 & 1238 \\
348 & 2351 & 1175 & - & 367 & 2503 & 2502 & 1251 \\
349 & 2357 & 2356 & 1178 & & & & \\
350 & 2371 & 237 & - & & & & \\
\hline
\end{tabular}




\section{Appendix D Factors of $5^{t}-1$}

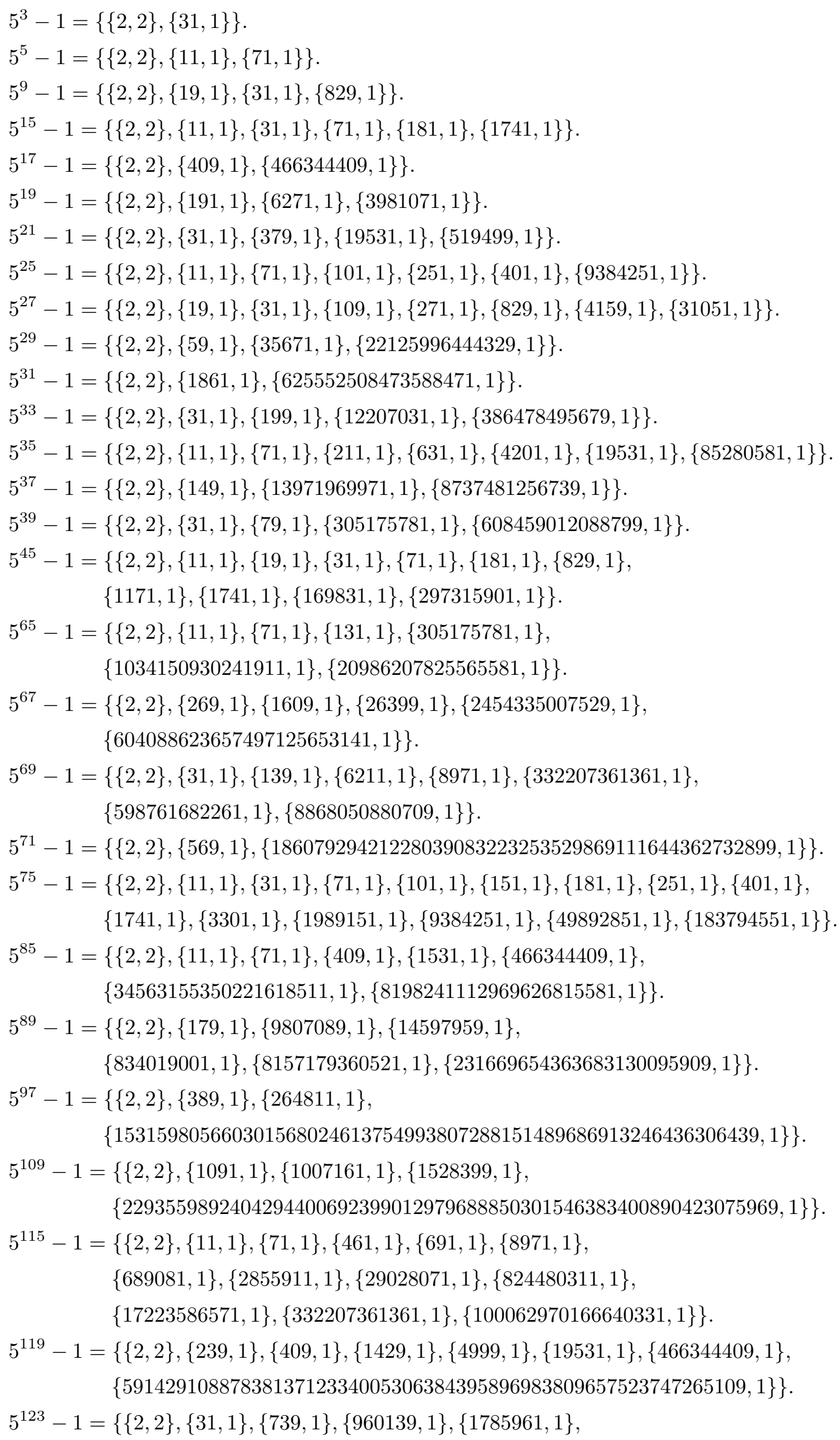


$\{2238236249,1\},\{9025583299,1\}$,

$\{29624915776321538277133753064212177050469622587571,1\}\}$.

$5^{129}-1=\{\{2,2\},\{31,1\},\{18471511,1\},\{1644512641,1\}$,

$\{172827552198815888791,1\}$,

$\{2257128352803905294127694161479357934346533799518511,1\}\}$.

$5^{135}-1=\{\{2,2\},\{11,1\},\{19,1\},\{31,1\},\{71,1\},\{109,1\},\{181,1\},\{271,1\},\{541,1\}$,

$\{829,1\},\{1171,1\},\{1741,1\},\{4159,1\},\{11071,1\},\{31051,1\}$,

$\{169831,1\},\{297315901,1\}$,

$\{1312315694449748688331,1\},\{26941244373060650224561,1\}\}$.

$5^{155}-1=\{\{2,2\},\{11,1\},\{71,1\},\{311,1\},\{1861,1\},\{11161,1\}$,

$\{257611,1\},\{5624951,1\},\{31473312961,1\}$,

$\{237905328491404897095803500170782113740458997606786018097569551$

$7755589901,1\}\}$.

$5^{165}-1=\{\{2,2\},\{11,2\},\{31,1\},\{71,1\},\{181,1\},\{199,1\},\{331,1\}$,

$\{1741,1\},\{2311,1\},\{103511,1\},\{511831,1\},\{12207031,1\}$,

$\{65628751,1\},\{190295821,1\},\{386478495679,1\}$,

$\{134046379175988442036236898365227099024297688760741,1\}\}$.

$5^{175}-1=\{\{2,2\},\{11,1\},\{71,1\},\{101,1\},\{211,1\},\{251,1\},\{401,1\},\{631,1\}$,

$\{4201,1\},\{12601,1\},\{19531,1\},\{28001,1\},\{50051,1\},\{200201,1\}$,

$\{8894201,1\},\{9384251,1\},\{22661801,1\},\{85280581,1\},\{1657309151,1\}$,

$\{38105263380318401,1\},\{16711449148651875290388101,1\}\}$.

$5^{173}-1=\{\{2,2\},\{1039,1\},\{3461,1\},\{1708272343265311,1\}$,

$\{339919831020187630960347957199421099342434386629129924807724899$

$548171905079254114627192212342747249,1\}\}$.

$5^{179}-1=\{\{2,2\},\{359,1\},\{3581,1\},\{75539,1\},\{17315029,1\}$,

$\{194033876682173390072729445439967121968705407607468704063229337$

$00153368084799315563561578033131860319559619,1\}\}$.

$5^{185}-1=\{\{2,2\},\{11,1\},\{71,1\},\{149,1\},\{2591,1\},\{30810641,1\}$,

$\{13971969971,1\},\{8737481256739,1\},\{21829321837586441,1\}$,

$\{205921274392755938824062408247800624179086850744953308815997462$ $39870280151,1\}\}$.

$5^{209}-1=\{\{2,2\},\{191,1\},\{419,1\},\{6271,1\},\{56431,1\},\{3981071,1\},\{12207031,1\}$, $\{220779632976901561101225956232975832161904766968039073773434923$ $37000542711435241254425456657076461909793693026506147389,1\}\}$.

$5^{215}-1=\{\{2,2\},\{11,1\},\{71,1\},\{431,1\},\{1291,1\},\{144982241,1\},\{1644512641,1\}$, $\{458230898247580490693474825320925631629830454700386225782183042$ $2052712517500006897081991082494828288526639138845788438012251,1\}\}$.

$5^{219}-1=\{\{2,2\},\{31,1\},\{439,1\},\{429241,1\},\{2183431,1\},\{4853479,1\},\{5729041,1\}$, $\{836698497295359483931065860382921422898058342124230343108001332$ $286410112772295081638094714264104687084007799369368144246311,1\}\}$.

$5^{235}-1=\{\{2,2\},\{11,1\},\{71,1\},\{941,1\},\{4231,1\}$,

$\{20885861,1\},\{68308861,1\},\{96766610646500911,1\}$, 
$\{105475693151619035529055506571643366314164278037417148495742900$

$661283586936266520114117025199330220448787532328441826879351,1\}\}$.

$5^{239}-1=\{\{2,2\},\{479,1\},\{40093613041379,1\}$,

$\{147353459691520632244555607717478117134030802606181953708010371$

9298166168947549642538525464219037490151421698581086237756602026720817

$756235926209843391,1\}\}$.

$5^{243}-1=\{\{2,2\},\{19,1\},\{31,1\},\{109,1\},\{271,1\},\{829,1\},\{1459,1\},\{4159,1\},\{4861,1\}$, $\{31051,1\},\{14001661,1\}$,

$\{956225817178767724216036727013172355771961587713861458223950326071325$ $32203177149599172957989777513262960247318785445596060912523871806859,1\}\}$.

$5^{245}-1=\{\{2,2\},\{11,1\},\{71,1\},\{211,1\},\{491,1\},\{631,1\},\{4201,1\},\{8821,1\}$,

$\{10781,1\},\{19531,1\},\{85280581,1\},\{16650328910366149531471,1\}$,

$\{781663320776776898423953184448429313182200602330122956263346853$

$362609732059029620242463194363111463695774602408391,1\}\}$.

$5^{249}-1=\{\{2,2\},\{31,1\},\{499,1\},\{12451,1\},\{169321,1\},\{20515111,1\}$,

$\{413066447794038087591111753737021242470889068026169507364672570$

9170070986722837017231782732467782049914630406727484039932117694033845

$10804794807777014929,1\}\}$.

$5^{255}-1=\{\{2,2\},\{11,1\},\{31,1\},\{71,1\},\{181,1\},\{409,1\},\{1021,1\},\{1531,1\},\{1741,1\}$,

$\{90271,1\},\{236641,1\},\{317731,1\},\{466344409,1\},\{654652168021,1\}$,

$\{782302186051,1\},\{38385602257801,1\},\{263420722813531,1\}$,

$\{1445377105917001,1\},\{373669429273223813320308794167606428171892018391$ $7268996709350346031,1\}\}$.

$5^{265}-1=\{\{2,2\},\{11,1\},\{71,1\},\{1061,1\},\{5960555749,1\}$,

$\{17154094481,1\},\{488094322309591,1\}$,

$\{1335618937071191,1\},\{27145365052629449,1\}$,

$\{281247180648443474201460266274425491641129732331700920080143804$

$12159116372499099715505705982461473940474528773181,1\}\}$.

$5^{267}-1=\{\{2,2\},\{31,1\},\{179,1\},\{1069,1\},\{1133149,1\},\{1652731,1\},\{9807089,1\}$,

$\{14597959,1\},\{834019001,1\},\{8157179360521,1\}$,

$\{18484574880511,1\},\{369718501929859,1\}$,

$\{142567547569335926285313687359043351519843476238292654496637148$

$00094573110287775614743544597716721806051,1\}\}$.

$5^{283}-1=\{\{2,2\},\{1699,1\},\{451669,1\},\{113334709,1\},\{416742821994932569,1\}$, $\{443819633770294750370320744731630318561750138440580012523002611$ 2644708672382627319041587277968263957097948226651971357369795069152786 $800319216626055584292591152381,1\}\}$

$5^{285}-1=\{\{2,2\},\{11,1\},\{31,1\},\{71,1\},\{181,1\},\{191,1\},\{571,1\}$, $\{1741,1\},\{2851,1\},\{6271,1\},\{30211,1\},\{3981071,1\}$,

$\{4113691,1\},\{260930841421,1\},\{1245576402371959291,1\}$, $\{168114510382313038536111985312939538121149394255181710985204428$ $0971975267257975994205963362124950242977896917525215729402771639991,1\}\}$. 


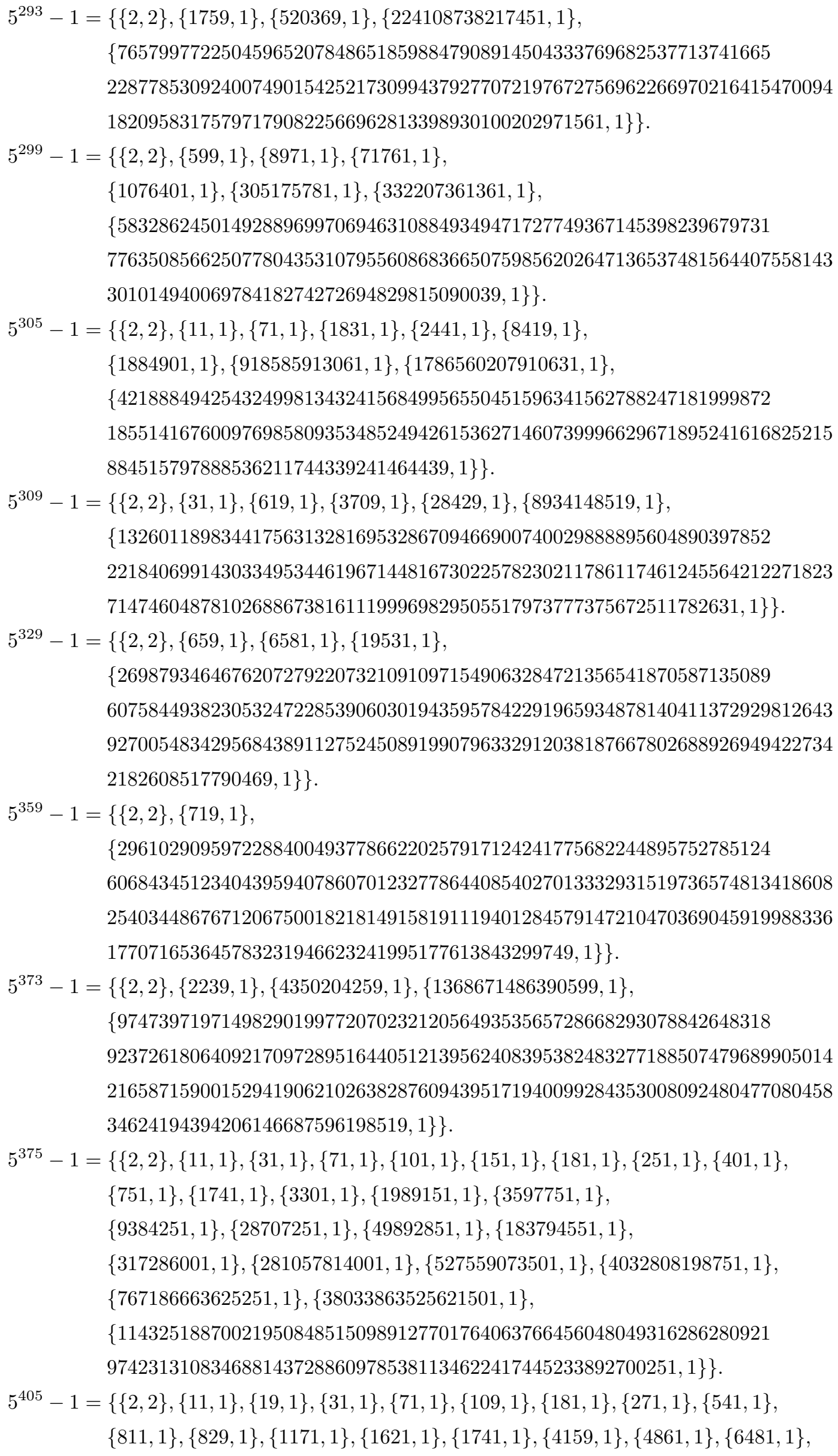


$\{11071,1\},\{31051,1\},\{169831,1\}$,

$\{297315901,1\},\{921737881,1\},\{2332368714077641,1\}$,

$\{209305668209401393906696586829173386548951703282782780402553460$

4281926648084538557320065865164192752664854338990368623633792347764896

$65502175622390165033543404704840479100212903257395725653743351,1\}\}$.

$5^{419}-1=\{\{2,2\},\{839,1\}$,

$\{220094841901924571650987622467147400187094486771311371516144911$

7140159759304200062656676660861113413530002784108123544879051065501207

5894299635120175043081595033434578406403276368642668179446972259374668

8082047236274409502287386645680602664196997296318792549098861502565421

$87002049015304329,1\}\}$.

$5^{429}-1=\{\{2,2\},\{31,1\},\{79,1\},\{199,1\},\{859,1\},\{12207031,1\},\{17885869,1\}$,

$\{300128401,1\},\{305175781,1\},\{386478495679,1\},\{24615836446631,1\}$,

$\{608459012088799,1\},\{53130566763791958299,1\}$,

$\{700396364054946116190231244187233697567991757371291316621476117$

6799006842906628885334213443937040728444168526156733697430270471385354

$3661941031239492658681225854942026969634862817879672867458561456299,1\}\}$.

$5^{455}-1=\{\{2,2\},\{11,1\},\{71,1\},\{131,1\},\{211,1\},\{631,1\},\{911,1\},\{4201,1\},\{19531,1\}$,

$\{4914911,1\},\{6481021,1\},\{85280581,1\},\{305175781,1\}$,

$\{1034150930241911,1\},\{20986207825565581,1\}$,

$\{146684795895102231260166784920352531108675064294400608294885034$

0905470330356375848939977489571716625611881592741713102101551752050402 3924813891216562301950476364388599231332757591866687441936062297396840 $595073147763297541261866796998261,1\}\}$.

$5^{459}-1=\{\{2,2\},\{19,1\},\{31,1\},\{109,1\},\{271,1\},\{409,1\},\{829,1\},\{919,1\},\{4159,1\}$, $\{4591,1\},\{31051,1\},\{90271,1\},\{317731,1\},\{26481241,1\},\{261594199,1\}$, $\{466344409,1\},\{1203176701,1\},\{654652168021,1\},\{360143672909416579,1\}$, $\{198791992856326005085331376433482969605877318722143940889431980$ 4894637205096730266048719884836781508200445907446177055541463223091905 6968448792217866979062634356900821261150046787250707621508329544486830 $56984579063792129,1\}\}$.

$5^{485}-1=\{\{2,2\},\{11,1\},\{71,1\},\{389,1\},\{971,1\},\{3881,1\},\{12611,1\},\{264811,1\}$, $\{2387171,1\},\{5829701,1\},\{11401381,1\},\{4588270721,1\}$, $\{899097422777008401851167409568439226688354258115294906618122747$ 7825324926036222787025968368592102291927703447093449702450262771318706 6249071410259549284341748679710143473042823510898803659134671330264377 6133966452350877715496313019175075477150523615095205796302505513649244 $61716018167949,1\}\}$.

$5^{487}-1=\{\{2,2\},\{1949,1\},\{949651,1\}$, $\{338030782923115295201942716301325798939609625814210230551939611$ 9450153312011888037389389385947409468465847668583884693195586019619386 0817165008766874819376951558998414740283961760882813466887725792278756 
3509269167736157038164038007165920417813999991264751741881801113872837 $0889507942642873762460448033520076465197895766637434176669,1\}\}$. $5^{495}-1=\{\{2,2\},\{11,2\},\{19,1\},\{31,1\},\{71,1\},\{181,1\},\{199,1\},\{331,1\},\{829,1\}$, $\{991,1\},\{1171,1\},\{1741,1\},\{2311,1\},\{19801,1\},\{103511,1\},\{143551,1\}$, $\{169831,1\},\{511831,1\},\{1731511,1\},\{12207031,1\},\{65628751,1\}$, $\{190295821,1\},\{297315901,1\},\{578259991,1\},\{386478495679,1\}$, $\{14300545887541,1\},\{22542470482159,1\},\{153560376376050799,1\}$, $\{471311128435705185395333851591707750513327503195414560148636789$ 3181842375952341924523640846996910941087490582379418488012396472231982 $85908481922810825497154824184211889638713528318295628721,1\}\}$.

$5^{509}-1=\{\{2,2\},\{1019,1\},\{219889,1\},\{3600024661,1\},\{1258198860123384481,1\}$, $\{146973746295196128684452452818361140606771984634491835822894983$ 8437884345459056777706312251825375554709899720188741443637459551194465 3088447227394532932093811045757366103850647757673358506700903814558120 7495055129900688439935425670675031729380609285642570582070360912250188 $85530147348349879074925378708005351331363730851,1\}\}$.

$5^{515}-1=\{\{2,2\},\{11,1\},\{71,1\},\{1031,1\},\{3709,1\},\{28429,1\},\{41201,1\},\{96821,1\}$, $\{236985509596761461,1\},\{326493403069610501,1\}$, $\{889386641705408789383971855246449676478294659052010668700163546$ 1202167877843986323894152311145359968823939470841480436415600261268441 2231356140285550468181177445891205264842788328993576535458329913616307 5737362864547921627805643054962647695001425717452682239655975646107070 $5556286550555820914760842281,1\}\}$.

$5^{525}-1=\{\{2,2\},\{11,1\},\{31,1\},\{71,1\},\{101,1\},\{151,1\},\{181,1\},\{211,1\},\{251,1\}$, $\{379,1\},\{401,1\},\{631,1\},\{1051,1\},\{1741,1\},\{3301,1\},\{4201,1\},\{12601,1\}$, $\{19531,1\},\{28001,1\},\{50051,1\},\{200201,1\},\{519499,1\},\{1736701,1\}$, $\{1989151,1\},\{8894201,1\},\{9384251,1\},\{22661801,1\},\{49892851,1\}$, $\{85280581,1\},\{183794551,1\},\{1657309151,1\},\{37815352051,1\}$, $\{119461537021,1\},\{21226783250214361,1\},\{38105263380318401,1\}$, $\{238058276429599046633889495325950582786109822001395692046300078$ 4429772902260954842067760053141367248182478411305061359649046387270446 $29087849693296660874466713715197797393692682501,1\}\}$.

$5^{567}-1=\{\{2,2\},\{19,1\},\{31,1\},\{109,1\},\{271,1\},\{379,1\},\{829,1\},\{2269,1\},\{4159,1\}$, $\{4861,1\},\{19531,1\},\{31051,1\},\{280729,1\},\{504631,1\},\{519499,1\}$, $\{2161279,1\},\{576448489,1\},\{19523494474219,1\}$, $\{23792163643711,1\},\{305921358183421,1\}$, $\{261192068102536376507282642127041664344171607867105650924528627$ 4596750554018456723970355767575097657709712607823360174583088569608923 3922967208332295114985706667211544260659083539213150528502893820571290 9452582033133573304440459720050238572577147519276397563917711499090275 $245563536244218079,1\}\}$.

$5^{575}-1=\{\{2,2\},\{11,1\},\{71,1\},\{101,1\},\{251,1\},\{401,1\},\{461,1\},\{691,1\},\{1151,1\}$, 
$\{8971,1\},\{173651,1\},\{689081,1\},\{1069501,1\},\{2855911,1\},\{9384251,1\}$, $\{29028071,1\},\{40231601,1\},\{201781301,1\},\{824480311,1\},\{17223586571,1\}$, $\{26135496851,1\},\{332207361361,1\},\{100062970166640331,1\}$, $\{776336687349790478683146353378325024509295187614020013470753591$ 9805002280206672322343575762994364780445829682469859194831432195034544 6172559108807780088047953275092657800931383604622064865008749807192276 $9076734850490448561395449553163289465447181382337609120985430951,1\}\}$.

$5^{577}-1=\{\{2,2\},\{2309,1\},\{23081,1\},\{185750794563808939,1\}$, $\{510532361770087092677610369775809716832374342780259549054971774$ 2124893690644995450992445164854173535740877942826040901048177544675143 7580506967315106981145306893158190984187824850132155627924424389614166 6600492281665066313062961310952533955731856065326527481880479798198289 0021309195271550512997431030529858391463628455972943672282802764051571 $84701335010821741499778466823081551,1\}\}$.

$5^{615}-1=\{\{2,2\},\{11,1\},\{31,1\},\{71,1\},\{181,1\},\{739,1\},\{1231,1\},\{1741,1\},\{15991,1\}$, $\{62731,1\},\{248461,1\},\{960139,1\},\{1785961,1\},\{2238236249,1\}$, $\{2908888501,1\},\{9025583299,1\},\{18383372131,1\},\{1256950067521,1\}$, $\{456484970171877894901989894933894898942574834047907321434076484$ 4336840124334879270913831344323062846599274786231684269862095407509136 8429721039883744204100156921134385130885137644882818628077143058175378 0799510654162406823735374363119666524638907529637669605204522000923314 $602201807870072259651929641024743419704239183571424195075830011,1\}\}$.

$5^{629}-1=\{\{2,2\},\{149,1\},\{409,1\},\{1259,1\},\{94351,1\},\{9895429,1\},\{466344409,1\}$, $\{13971969971,1\},\{2135840614741,1\},\{8737481256739,1\}$, $\{128835865446894588142534999730360282876025533774092105693220606$ 3583181903622535809458323281611123060456914577332640166721834435375558 2682696973764171794240522314293748479753077359269414736447573007517612 8848878862980382978292383611919560838398187108302650498036060196950888 9263158799944805231357810168384171639932610148809690802333388615734964 $360402793563340053161645626478021,1\}\}$.

$5^{639}-1=\{\{2,2\},\{19,1\},\{31,1\},\{569,1\},\{829,1\},\{1279,1\},\{12781,1\}$, $\{156309592445437909,1\}$, $\{154372407640178035972718959849855510075851291129195424555269926$ 21511807997024881018691911700160086751558867454940858883369535880181037 6574225878317083666478751008127311387396308176011881133512689090922868 6903644013894877500614888718197308144346730221874873185264528060083209 1968451930075236859610433292856547181624398200251821438426075272184672 6943438867060846545462387370375740538769557305573684041489509036225466 $9,1\}\}$.

$5^{659}-1=\{\{2,2\},\{1319,1\},\{146299,1\},\{4706579,1\}$, $\{115075099807935588882021264751940094056155658637463377663853667$ 2206325203222639995590991098386948972406876806450043309798100862551290 
1292522223419502309561907598083365569206790474080180532330333932089730 5234632825645985917158221618002412810184902524062641497304214529600271 0875872724080886540431482317383576698771720528781378676437790220381565 5720738331175622319741590097048106646130065955094232814718643685476637 $413014100006068869226112620421369,1\}\}$.

$5^{699}-1=\{\{2,2\},\{31,1\},\{1399,1\},\{6991,1\},\{258631,1\},\{1318781,1\},\{412500871,1\}$, $\{222831302398955532521959190646940823113894144155108483002198117$ 7501325222743556885240494533503982712196587074139199532772400503560172 8394673583737092361870300281877823040182496957960817930837500717198089 1287301747160520803485484678661057684054072997657392338164758307317505 0185369187191980829718153530185588994084797701815317910747398890149993 8540963893977695560902086517433868908057745827399178550040033902568727 $56233797854303188005365115119685364392259235269,1\}\}$.

$5^{719}-1=\{\{2,2\},\{1439,1\},\{8629,1\}$, $\{730048752772368934341123593939801606098928950940927711629242732$ 5701371806504719514940286879758892289909895020967193984118283825131897 5095217791119534671414904910299957951194155009452322926943008430294331 5738406779650578446298115081840384401164237856813807289330906718730121 1258167224216416885377153479655206689197604845471750147165436960489423 4260556846443417973787106568044080428710483867270480445839244758346117 4698387105966302027813502158311672480998820250194897966997959158393251 $419121375401,1\}\}$.

$5^{725}-1=\{\{2,2\},\{11,1\},\{59,1\},\{71,1\},\{101,1\},\{251,1\},\{401,1\},\{1451,1\},\{21751,1\}$, $\{35671,1\},\{97151,1\},\{1461311,1\},\{9384251,1\}$, $\{22125996444329,1\},\{508005163062054101,1\}$, $\{179361077822467174056605430720587201104180499067236863659757258$ 3246139207142222047982008477792446003969837501791733658935837497792483 4949686678977493215711816837355472847487615780543460344094576227386180 9144537104855211973547740874053104678976331674031690255482831339642669 4651516642482203138617240398608712932655656196080363400941650616538363 2684607155281790885121592091352815909222972036726964724382069134619985 $920232193635928117761,1\}\}$.

$5^{735}-1=\{\{2,2\},\{11,1\},\{31,1\},\{71,1\},\{181,1\},\{211,1\},\{379,1\},\{491,1\},\{631,1\}$, $\{1471,1\},\{1741,1\},\{4201,1\},\{8821,1\},\{10781,1\},\{19531,1\},\{72031,1\}$, $\{519499,1\},\{1736701,1\},\{24556351,1\},\{56117251,1\},\{85280581,1\}$, $\{5071357999,1\},\{119461537021,1\},\{2035895040229,1\}$, $\{12388179201301,1\},\{21226783250214361,1\},\{16650328910366149531471,1\}$, $\{154576006904264792879340085445529610458109505205649255745195165$ 8399419576649313649300949233360011187902444795237240695638475373909128 2561586945544386967224867234036578784031410017827558434736486240113130 5918682029223872264116724727648750987159953478866870339784015222436398 6381771660879301476579224437916357908652304765272369996456557080301743 


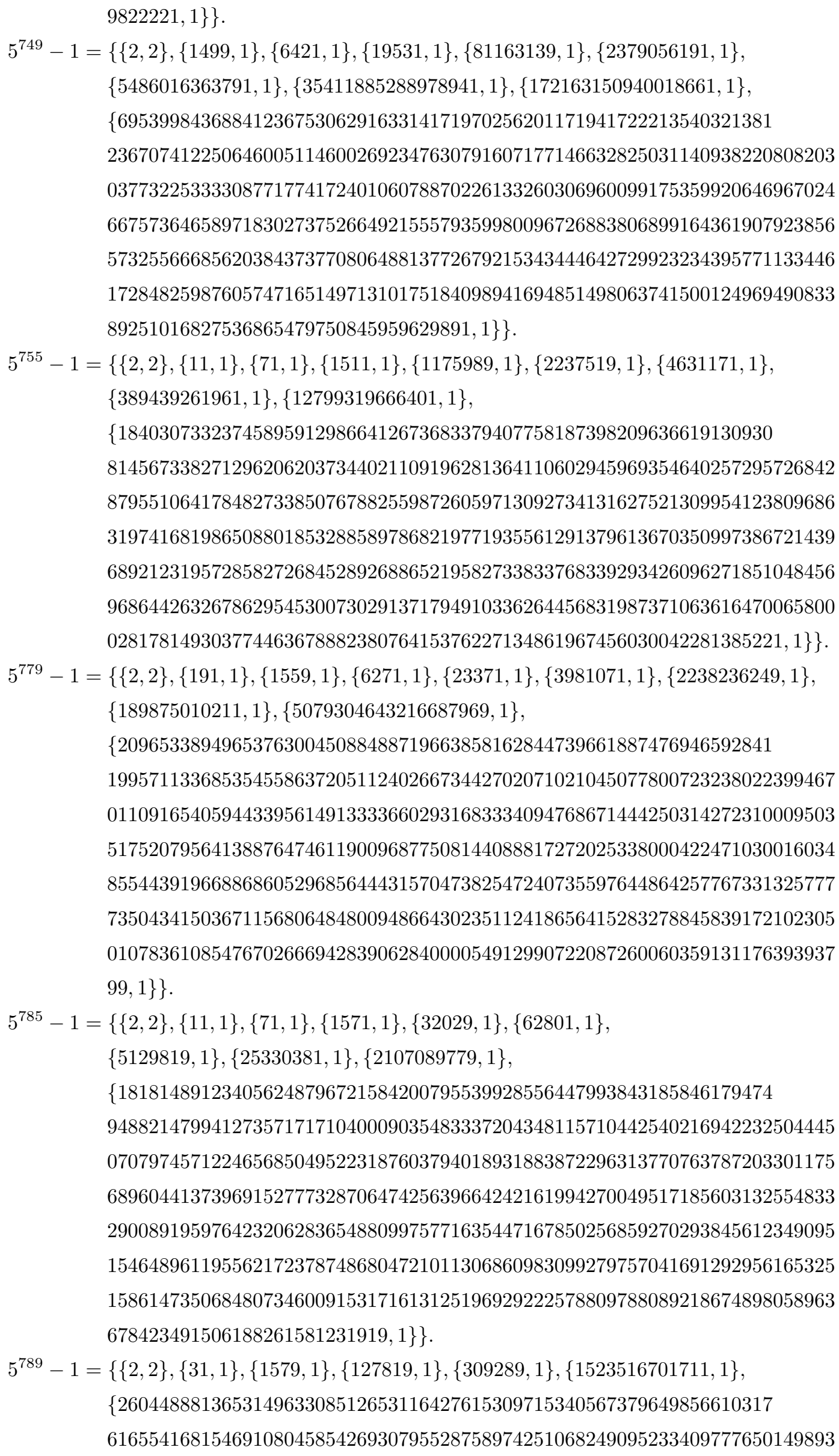


0577001227146142902326868045250100042253239547899214321626257217774505 4713359072744630844551955425840845648209119551734235805551254337392960 6784490187219454965113266542835995143071639348870413160991647706274452 9064774339803341614648212637196013044426089258739711079599730520793795 7308731697654480867743431049535833076461079748257780288084171159526454 $35097687870689614922710033346996338673069,1\}\}$.

$5^{809}-1=\{\{2,2\},\{1619,1\},\{50159,1\},\{397243271,1\},\{6516689161,1\}$, $\{348332697327606093148604562260564875908744361209971991539247938$ 1073650113837882192773808367170996510645200155426520255832304875377481 8230763448967827523961732561765942962808927515197117499871228430944122 6649438817659900591483058480624118315701834824277208320476077200739265 0021758031574544664295768690309439020727517790383887153829943837861103 53786717412708468597039768878672652022467671026270938855570893896881394 8054030739436355498384076152145928393029869673863327514926153308418690 $56022797844853293535387850190719558917122659497513826131,1\}\}$. $5^{905}-1=\{\{2,2\},\{11,1\},\{71,1\},\{1811,1\},\{1576511,1\}$, $\{700931551,1\},\{478171613586061,1\},\{10906993756490561,1\}$, $\{113386925240786316809501508104157064516108786378678418078872228$ 3483062969267936887682647720492356427291921625892962160394553562902800 2352166378273782439776027551591276863597125873525940375623631222669590 8317488314654419698518671406379907066458892518546825848071613924677390 3062876730878664939940225532110918846008291014101034102826997047771592 3268604153995893310679605616607624297094601170492449800711879085240809 7106898647323521268128439263577987062511524000680499145886478418391800 6863072688827556603943390883801698080722504977589556175158258719160307 $7979787718613621692095064011,1\}\}$.

$5^{935}-1=\{\{2,2\},\{11,2\},\{71,1\},\{409,1\},\{1531,1\},\{1871,1\},\{103511,1\},\{192611,1\}$, $\{511831,1\},\{4200769,1\},\{12207031,1\},\{65628751,1\},\{190295821,1\}$, $\{466344409,1\},\{53289827581,1\},\{1497355422751,1\}$, $\{351678366803441467892309697922410370627065083280387860269554806$ 4581904675475181281091167986329066013504927126276950771308858021188085 3443314230103626183962925366462840336279850862571459945455910778479463 9540406066785051791512230980737780195550854979952715899281959393891202 5085419793173371786651223197242155610694816370122284300826099709248450 6761061060762641307527660737826289166643154560060901898863704606355596 2645997908443152199988046833641459055143430974924675078912148567415433 5858877996845413465208312032312489840104474253321366178396521439673350 $9002346549,1\}\}$.

$5^{939}-1=\{\{2,2\},\{31,1\},\{1879,1\},\{229651231,1\},\{37421136592412809,1\}$, $\{107473119382166344580764931256482489517336196975297028974415854$ 8039237126956598691612184079420046616200019364883071718530423973956830 3491847575395277005226186685775803518061921567587573021112749975531419 
5876853515105575422709466710370352212018261971658049222452520739443502 2705840159707524094963475279126454154234900940126398582995963343457990 7329909893259476756577048238039228436906629354322765596506775301294650 9513563275356987502131757213787912310633244347192397551539210840540433 1410033560842965545883996467272943173870527436180991779376081873171578 4456650154972609033956227370737008218970700549883482566225952700991146 $7961,1\}\}$.

$5^{965}-1=\{\{2,2\},\{11,1\},\{71,1\},\{1931,1\},\{36671,1\}$, $\{295291,1\},\{1370755481,1\},\{749154725101,1\}$, $\{478031381057805845194338121164306039051947799182178207225303630$ 4850370021121144626967595938200220908105521396176240886703648967799679 9529641927610840730115173590104656823728658593829752924089720360131127 7982991675889323812453524194215241723300512771984291137281387991586485 8005725694204224853875000215233086586732252624033884312598852889068698 6149682175799115640355128445589656358912081928555554292995555841464744 0955325379073222391521790839453781132401241722723893692879942110501841 3845028738259322656083428226179113874236928635728804950602075491879140 7572828733413119566197309328951129043621981283232598353808112844291831 $66715947107131,1\}\}$.

$5^{975}-1=\{\{2,2\},\{11,1\},\{31,1\},\{71,1\},\{79,1\},\{101,1\},\{131,1\},\{151,1\},\{181,1\},\{251,1\}$, $\{401,1\},\{1741,1\},\{1951,1\},\{3301,1\},\{14821,1\},\{17551,1\},\{107251,1\}$, $\{1659451,1\},\{1989151,1\},\{9384251,1\},\{49015201,1\},\{49892851,1\}$, $\{183794551,1\},\{305175781,1\},\{933692761,1\},\{608459012088799,1\}$, $\{1034150930241911,1\},\{3879644432625001,1\},\{20986207825565581,1\}$, $\{142816611356873351,1\}$, $\{123850673278068987633010284192169839273122959719985297727636915$ 2555509239608205272717222422489532992990383902490582335996609093376089 4998751121826134250921655624067456494368160176515085989002475125564243 7076318409387913654975682997635264156215815530059761699975190368601159 5763519229195641703485715570189856084639570896488105298903083243306117 2263624645207026611266834814525143594194915890358668911119031823796487 7997430526580714121651634957345583693411786187541585122387562067422016 $4994672029728251,1\}\}$.

$5^{989}-1=\{\{2,2\},\{1979,1\},\{8971,1\},\{1644512641,1\}$, $\{332207361361,1\},\{8345878028531,1\}$, \{590295054674310093578012584727683108754458904079262716199606752 8573920869388812633714385114515020248410873951986034322638825921027042 3088354498466371567427243009899475242376276499749694558070577494626376 1298325977546834529414887353818911998912301998464114116857014960004227 3697159524546423738010567274095496361259295331292271902559367668116880 7441042370774960283326819619082573310919367880212160299113972001133360 3183854871049672464981430581854287294367479232742888495335636414089431 
8491223420842162546933312223547268698997347054902847094218958601219464 9962813507055665638644724530194971806814350774262546050724327752475443 $668258823327416118774099439,1\}\}$.

$5^{999}-1=\{\{2,2\},\{19,1\},\{31,1\},\{109,1\},\{149,1\},\{271,1\},\{829,1\},\{1999,1\}$, $\{3109,1\},\{4159,1\},\{15319,1\},\{31051,1\},\{344145511,1\}$, $\{13971969971,1\},\{966739015279,1\},\{8737481256739,1\}$, $\{434809233528041113653416827379371108979955050516301210232063963$ 1124204598589716888398392755428616230226668224059176988475689602245467 1871190019101495256757495956664354892722143753317869611857118066336524 6704987291976313467047782831696890312730698425294021762596469390292092 5730018139576476971806439440455225968488213104962677500279076595124533 23220565109085888493820603662819038711145323161116775219361971106954593 5321887064833740491738372559140737515652416818059530764394957286121899 6695182344245409183515320167308174718663303309173343809352893406600873 6252533296229266753719073823896349201046169342781948376016332230633821, $1\}\}$.

$5^{1005}-1=\{\{2,2\},\{11,1\},\{31,1\},\{71,1\},\{181,1\},\{269,1\},\{1609,1\},\{1741,1\},\{2011,1\}$, $\{4021,1\},\{26399,1\},\{126631,1\},\{5031363661,1\}$, $\{2454335007529,1\},\{598266261966511,1\}$, $\{110562601576528691339722276681709205161002122577562897413801590$ 3041055751095244566605372323402876447302420334083732561434566356269179 8857237126024490213052800329811796443634154541569511784715631881977604 1161942758931412495898115378039723411731572625857461082438216286013516 1138746891490408409832163961307278664049986838088043723917819046092511 4716571005678499798725578270665587324459806607359042789421610876308512 8492880002588483272737868689150485494567886437779334052055143276306049 3615717742208098273901785365766190630797753068351500638591766782933001 1714138096700565840208620492162367177110621603675602015521048706023775 $39542514431,1\}\}$.

$5^{1019}-1=\{\{2,2\},\{2039,1\}$,

$\{218251481952643588336462257707935661031801469393068350225927162$ 0291519798738517112167026149104964218307079293507636891618589930516482 0599400255148458830003149885022006199140073365474717435837528905557283 4423001837587873982423408594081231873341882410982696580642324731329943 1209715424031173013981118145612143650421825321628108467357350313990718 9169249554840422616636115668792075667997941626245214414823824341373149 9902997887818871374544147769791993306723621935437124636032454352410852 1473315200403538610997034882591162190528798454302547740675396926091751 0095979251758243275165878595182900732784434755606542314695194387825864 8954661498575071962692980980026394841210050766915389767367855878468329 $2997473884646129,1\}\}$.

$5^{1055}-1=\{\{2,2\},\{11,1\},\{71,1\},\{2111,1\}$, 
$\{20876816861,1\},\{1532829720131,1\},\{5594292675731,1\}$, $\{219407737270108201362995646029954902902692811181716394394141557$ 8897791667026346743252530018606588533820299322215327694018977690165000 9173449405691290292378406182498652060704532138881124645901655921473994 6302922508477355891132190856558570900028667520590042821747097119378328 0120453989331371954532868744179110034689886950915998822371601123714461 6321733211733736832505853481171515886570736806445726731080478459971272 7426451979415396372143069095397095208132280131277916217072945194140186 7667976961017556426739212219249048014588408986154312806593814664967370 3114382692054067817258013257812492542831350168718947387359794305447169 5483320912352084672632576855803565323547866623668299693215828201453642 $621,1\}\}$.

$5^{1049}-1=\{\{2,2\},\{2099,1\},\{472051,1\}$,

$\{418285872447881836127086617462267904694651105412092223380622808$ 48026668520593797621780223578888666503717210048931901995448480748982614 4809937876283623616678794991952016363843529252502700484864600978199900 7604425992457320014320715811181247036335360078703966881870020644368190 2261614443395841785572117142468056868207890714401635095531258872880984 5062184634282685622235455861810627389432680989330209038396750044717979 9536845878113336537130706684493891933273682221416470729895877630713384 8114481182282042172604656617869577297194309832407937625776372316802220 7949586559844548170539456795112945255333208611141534358205856277017706 8141575643182375281125000087358553933290920295436444407090952541885559 $3065230438086583447703638635169,1\}\}$.

$5^{1065}-1=\{\{2,2\},\{11,1\},\{31,1\},\{71,2\},\{181,1\},\{569,1\},\{1741,1\},\{2131,1\},\{10651,1\}$, $\{17041,1\},\{308851,1\},\{7484111,1\},\{8499626476216091,1\}$, $\{156309592445437909,1\}$,

$\{172738969156675153767554919928674301908139060841753166306164421$ 4189690123369378716610640063781794383565296376179134213098377939343412 2938358104966483627821570306090501776543835254737755203729273882552483 5087219377475874616098189001424373840184860240057527724132469969295370 7067572593162538139920611019617804562558081809511240642220870834243054 8836378595903422604791634167638102083478243042208574401147355783100718 4366499528617149355921769093354447482417100135630692353617760131813785 4681291328528104759469997970277925002438670435929251074098481597257894 6779869907717181891577228326912313981696581420287039081057456835228870 $07942904717760067893088665762897745112166019678391,1\}\}$.

$5^{1089}-1=\{\{2,2\},\{19,1\},\{31,1\},\{199,1\},\{829,1\},\{2179,1\},\{3631,1\},\{12101,1\},\{143551,1\}$, $\{486179,1\},\{1731511,1\},\{12207031,1\},\{386478495679,1\},\{22542470482159,1\}$, $\{315269911213473908876921694295763790765675764885015556112624070$ 4717104709342605504350627120321473849763044659567696037756471025274879 1271313429109795875682450977300504909593812851096269871559624188175367 
7593455432584442910301511639035210942217893094070012718879922636442627 19125164640846371065757394209552710924877102527168688888150435521610753 3010226847414439969521320715641295569168214391604793063309020454642278 2757470131476772693279111176795139536531904896751108650399074543385032 3958674637146061230669792512226263620148887674185118941338827527635590 1414531874744431757959291215382151529721120117611802635175360141647833 $1688882443590499211505497038162629043763429520830311107319424895362619,1\}\}$. $5^{1175}-1=\{\{2,2\},\{11,1\},\{71,1\},\{101,1\},\{251,1\},\{401,1\},\{941,1\},\{2351,1\},\{4231,1\}$, $\{9384251,1\},\{20885861,1\},\{68308861,1\},\{61909208515151,1\}$, $\{349569600611951,1\},\{640359292705751,1\},\{96766610646500911,1\}$, $\{365133874274123109588983278736440576978269837005094163089889204$ 3536974003444866851684254629249103727380093443470313102631007721295057 1758562029372433780176321852994174821320240867623898123433437513903238 0007616303229027376454441490740662030827282421051234521495964353477590 4823730922362420098726752327908456766998142791691211781341617562323349 2300819617095446631436215968809837585477736952200251886511230568785824 4825744318828320504209402422755090049410856749405895889740889293391568 7152278157978047563065698781578994209666630810242856105993506058224695 8313377804245463699925731017945073829005760138325373541524229928629466 5907416116951015354899986800455439045688046860176735606387346821661710 $26620332678059993786349151,1\}\}$.

$5^{1185}-1=\{\{2,2\},\{11,1\},\{31,1\},\{71,1\},\{181,1\},\{1741,1\},\{2371,1\},\{12799,1\},\{73471,1\}$, $\{190391,1\},\{657439,1\},\{19834531,1\},\{60098461,1\},\{68175421,1\}$, $\{38701052851841,1\},\{205367807127911,1\},\{809159522736541,1\}$, $\{16961361791934811,1\},\{104449388937445035371,1\}$, $\{241329614382387570241936215294982443103561047918220991508803358$ 4225183148008332996501517989971915105707914904179177309791936416269013 3979479132009878261479394937124012279598271936502418888452261414707356 4203639646818944721841430283204815337681758909697111906273188576549342 0292124816222693731221049708230173966340539871035791735592888206633080 4210183379532412145805659889820693536108315346304904076345485371127802 2520165654913040420342240947870724327081957425961070483010524253389127 1391226393719469721163109253894579717257468128488932919009218111107306 6167831058185933286421291813961812334752605936563683471314543850014035 1380513276369843819703726870494730684196332019144095185838065126791681 $, 1\}\}$.

$5^{1125}-1=\{\{2,2\},\{11,1\},\{19,1\},\{31,1\},\{71,1\},\{101,1\},\{151,1\},\{181,1\},\{251,1\}$, $\{401,1\},\{751,1\},\{829,1\},\{1171,1\},\{1741,1\},\{2251,1\},\{3301,1\},\{11251,1\}$, $\{169831,1\},\{1274851,1\},\{1989151,1\},\{3597751,1\},\{9384251,1\}$, $\{28707251,1\},\{31248001,1\},\{49892851,1\},\{183794551,1\},\{297315901,1\}$, $\{317286001,1\},\{1086749551,1\},\{281057814001,1\},\{527559073501,1\}$, $\{1390332823651,1\},\{4032808198751,1\},\{84244323125251,1\}$, 
$\{767186663625251,1\},\{38033863525621501,1\}$,

$\{161401612671764154910832523229158125157546315838504827258457148$

1474910287081516012227880722551166360794595919863514608094274368604155

2443617619334863005653111406455236842706768817381258793627306546556705

3789806231080979052436204562441927363272625809263342878928786429859934 9617364679411203587647877664130775288140742395408171387881770194157353 6300308518333261680847069904031262584473039226004909299010171480960899 3236855850639688332905788212477953531402005816340016940079169826481167 0758794249297492030701533132607914209012759108488326629782690583697698 $300758777951,1\}\}$.

$5^{1169}-1=\{\{2,2\},\{2339,1\},\{18371,1\},\{19531,1\},\{53441,1\},\{7330265231761669,1\}$, $\{948403860842441853681488418640586385531042275970744491555365726$ 1557315644654671962363511536602916400568413551235085603996648878809260 6046989882860679515071153389280913702501549482012599257767089136901935 0968707884274806035890919288681307665724823124949518092399305489722315 1027031607895625990981823450080108803188517429020235394272912244071542 2303311464701629460952727929732447571526317278769298373519254910699596 3222580663870706239898292057660925122511654059364839291367397387025261 1744438856732427097828914786437684294094794750623117198522715186132854 3054055105634265433218298753000236570135539480983982265237480663214831 9861847945752461245423618991900358541786676673925198739232183178552690 8274731433703586533434252032620509609657558060758833369156013544338796 $994588672709139165951,1\}\}$.

$5^{1199}-1=\{\{2,2\},\{1091,1\},\{2399,1\},\{1007161,1\},\{1528399,1\},\{12207031,1\},\{222354551,1\}$, $\{265539108941289292781121643363950775062829179497625215200686584$ 8887506385512110752895677678120337706686843668535045127758226584346923 8335822438836266124984906578163384928322694181882343322465723495365405 5969222576327085576354843095337575474817635361422846380594663235724686 9730220003273939919898393764326059382206825643721545992429061093875873 5469321368871758434275198671659226405229574772481492022736195252271148 2572863544100193516093204438261321459379745140340061028099575036456954 3521652787353917851744993498191366304191779238230131222256446255742143 3979171980414888407889130745202934611287415666286369269445252690491855 7371483914415101534891373696480285322209222349138250487081684180932943 3469982879915563978630671083436025361319873035634176642114839243303074 $50169625584181629021042313082041168178601,1\}\}$.

$5^{1205}-1=\{\{2,2\},\{11,1\},\{71,1\},\{2411,1\},\{79531,1\},\{578401,1\},\{882061,1\}$, $\{464913101,1\},\{26991752705081,1\}$, $\{2804771479250501,1\},\{19820497490365391,1\}$, $\{851271289277091156669646623080447333139093945827882767582922395178447$ 5912536575684313396165595504403328283623292210769697137978222553774522 7837296677465214477754759423724941660845776685995025712266869480264627 
3245548834810202797149320284877001333258994765524812949932893223050386 2632535386901322174053558547085059002795365672449587077724288574081679 4722017522092235184321700447891776234702194785015207790869176887232212 0657409966300237373762578819073013343453983603090232598258133053377299 1653957537554098411037884913381045657751547077562809419082919683542403 8597585222260816182698439306464078559104080846477441782190457383736665 2506594043108645746551934077661165322903925237445029665900383459938108 $157942821563595447279738701643702149237055679568309900692963655531,1\}\}$.

$5^{1229}-1=\{\{2,2\},\{2459,1\},\{887977841981,1\}$, $\{123855324096781217148195252177036890338938258043053688259927409$ 5136595666122657758579647259799758432570705386962476653361364732492506 1355349104197848169924339664696905904553125204426439922432658528257282 0395501904779952930209158161239168349964479049879204374198469192538709 8757067398043587418185044619198809720115407930948191511514847807041131 1629058643663814677795194268020918925671629860298071427335163237727730 9178551219861327383068651009022255856613042058680632377312902996575452 1960830899720230364318755945756207880228623296573012023714300928287445 9107733609461558952828386388500139488972468433864531865429068546791981 1947811561541393485111652629412862415140137486586470405105806587734733 1297270149177350301467306887640692095060168187321219461001926604604628 4089953962957102564114936913954058743274890516179436844050188994873386 $93573732139,1\}\}$. 


\section{Appendix E $\quad$ Factors of $5^{t}+1$}
(1) $5^{4}+1=\{\{2,1\},\{313,1\}\}$.
(2) $5^{6}+1=\{\{2,1\},\{13,1\},\{601,1\}\}$.
(3) $5^{22}+1=\{\{2,1\},\{13,1\},\{89,1\},\{1030330938209,1\}\}$.
(4) $5^{30}+1=\{\{2,1\},\{13,1\},\{41,1\},\{601,1\},\{2281,1\},\{9161,1\},\{69566521,1\}\}$.
(5) $5^{32}+1=\{\{2,1\},\{641,1\},\{75068993,1\},\{241931001601,1\}\}$.
(6) $5^{34}+1=\{\{2,1\},\{13,1\},\{1973,1\},\{20129,1\},\{45289,1\},\{12447002677,1\}\}$.
(7) $5^{56}+1=\{\{2,1\},\{17,1\},\{113,1\},\{337,1\},\{11489,1\}$, $\{2520917617,1\},\{37007700327977836913,1\}\}$.

(8) $5^{62}+1=\{\{2,1\},\{13,1\},\{8124604717965111529,1\},\{102651353523520067851969,1\}\}$.

(9) $5^{64}+1=\{\{2,1\},\{769,1\},\{3666499598977,1\},\{96132956782643741951225664001,1\}\}$.

(10) $5^{66}+1=\{\{2,1\},\{13,1\},\{89,1\},\{601,1\},\{1453,1\}$, $\{1030330938209,1\},\{6509387183417404924784917,1\}\}$.

(11) $5^{70}+1=\{\{2,1\},\{13,1\},\{41,1\},\{281,1\},\{9161,1\},\{234750601,1\}$, $\{13148832195720299503896018648521,1\}\}$.

(12) $5^{72}+1=\{\{2,1\},\{17,1\},\{1297,1\},\{11489,1\},\{152587500001,1\}$, $\{2739177855667309845605995807633,1\}\}$.

(13) $5^{76}+1=\{\{2,1\},\{313,1\},\{457,1\},\{21363981507860375753,1\}$, $\{21654472202540732126905077281,1\}\}$.

(14) $5^{116}+1=\{\{2,1\},\{233,1\},\{313,1\},\{929,1\},\{33409,1\},\{397823386118610689,1\}$, $\{668375848777845065895095866172488247660842757935193,1\}\}$.

(15) $5^{138}+1=\{\{2,1\},\{13,1\},\{277,1\},\{601,1\}$, $\{70942489,1\},\{22600337281,1\},\{66988220431117,1\}$, $\{6173240924263298830475044213655538296790181268066249595121,1\}\}$.

(16) $5^{142}+1=\{\{2,1\},\{13,1\},\{853,1\},\{72497533,1\},\{8367932201,1\}$, $\{200771218157,1\},\{554474665573,1\},\{1095832589179957,1\}$, $\{1092822132265806461035989014934429610237,1\}\}$

(17) $5^{146}+1=\{\{2,1\},\{13,1\},\{293,1\},\{27133517,1\},\{84128413,1\}$, $\{644660229355377335113807957224194811156238338635592348687079059$ $98833399876784982517,1\}\}$

(18) $5^{158}+1=\{\{2,1\},\{13,1\},\{317,1\},\{41081,1\},\{72997,1\},\{10390269663517,1\}$, $\{106574974400179673389676477182530542427426505210152936524875177$ $9411263689062240801237,1\}\}$

(19) $5^{166}+1=\{\{2,1\},\{13,1\},\{997,1\},\{4649,1\}$, $\{887141033302995056994713467520546276988118708626848063412547649$ $122524726582125833353427675039727600874069917,1\}\}$

(20) $5^{170}+1=\{\{2,1\},\{13,1\},\{41,1\},\{1361,1\},\{1973,1\},\{9161,1\},\{14281,1\}$, $\{20129,1\},\{45289,1\},\{14239201,1\},\{87393601,1\},\{12447002677,1\}$, $\{126360649744930309735312408866105725994974215298711571613719605$ $70761,1\}\}$

(21) $5^{176}+1=\{\{2,1\},\{353,1\},\{2593,1\},\{1827937,1\},\{2704769,1\},\{29423041,1\}$, $\{392043794951301890898248014184097556075760058828614220171074252$ $7266656440090784184414016132386689,1\}\}$

(22) $5^{186}+1=\{\{2,1\},\{13,1\},\{373,1\},\{601,1\},\{1117,1\},\{1489,1\},\{10269119521,1\}$, $\{8124604717965111529,1\}$, $\{126061025159642854132157189752003696842811740308347145923678374$ $49647876498016080563816161,1\}\}$

(23) $5^{198}+1=\{\{2,1\},\{13,1\},\{37,1\},\{89,1\},\{397,1\},\{601,1\},\{1453,1\}$, $\{6597973,1\},\{766142400277,1\},\{1030330938209,1\}$, 
$\{161015770297358190717594484428694459546167619869592876477497342$ $88071527463921911250763576406293,1\}\}$

(24) $5^{200}+1=\{\{2,1\},\{17,1\},\{1601,1\},\{11489,1\},\{25601,1\}$, $\{103390853395201,1\},\{909456847814334401,1\}$, $\{413358851438819728960350027504082170192096580314214059700983870$ $78334380286157790190547359523201,1\}\}$

(25) $5^{202}+1=\{\{2,1\},\{13,1\},\{809,1\},\{3637,1\},\{5728721,1\},\{3375268261753121,1\}$, $\{105174326056233443238401980067337481738107316832727246709094908$ $4443454114118856668976186837164720792055112948117,1\}\}$

(26) $5^{216}+1=\{\{2,1\},\{17,1\},\{433,1\},\{1297,1\},\{11489,1\},\{177553,1\}$, $\{39209617,1\},\{170735041,1\},\{152587500001,1\}$, $\{238654529871987179722647739647506102676996568195151715383257622$ $082030820162304344903519245682369139479265761,1\}\}$

(27) $5^{220}+1=\{\{2,1\},\{241,1\},\{313,1\},\{881,1\},\{21121,1\},\{148721,1\},\{632133361,1\}$, $\{224873451236210728258358609190892744857970891291803720297188245$ $67306261711831534960777195126498748181020732174835607000422936081,1\}\}$

(28) $5^{236}+1=\{\{2,1\},\{313,1\},\{1889,1\},\{66553,1\},\{38710282321,1\}$, $\{297249242512358547491888615800368810090644753770361512598340893$ 5193081812901050589463714219229769780015528104157404091135948335 $28895845309317193,1\}\}$

(29) $5^{252}+1=\{\{2,1\},\{73,1\},\{313,1\},\{1009,1\},\{6553,1\},\{90217,1\},\{390001,1\}$, $\{543097,1\},\{11824849,1\},\{1503418321,1\},\{2759159593,1\}$, $\{59509429687890001,1\}$, $\{819866248074619717120455482812290379487511077549784621303916037$ $4643036015305408138082028269249617715441177,1\}\}$.

(30) $5^{260}+1=\{\{2,1\},\{241,1\},\{313,1\},\{2081,1\}$, $\{51169,1\},\{632133361,1\},\{537181587281,1\}$, $\{989466084526315544694016787821734221726297373828571596209131430$ 4065297658445343396703549131884726806439116360332352366665113663 $745598178798333998289,1\}\}$.

(31) $5^{262}+1=\{\{2,1\},\{13,1\},\{1049,1\},\{17293,1\},\{48821081,1\}$, $\{586022872695362487415125212315099593756717010246775554400975007$ 0514328277914016286147107772387818856426543825163157869044664592 $0005215251236583389002007904038591671053,1\}\}$.

(32) $5^{266}+1=\{\{2,1\},\{13,1\},\{1597,1\},\{170773,1\},\{234750601,1\}$, $\{4885168129,1\},\{126286794969133,1\},\{2864226125209369,1\}$, $\{286729389765313125721925609608357082524297322817234204933292092$ $804164870508572541729524098355670110769591837945046868835094054837,1\}\}$.

(33) $5^{276}+1=\{\{2,1\},\{313,1\},\{1657,1\},\{390001,1\},\{3368857,1\},\{150220315444217,1\}$, $\{402297550539912605086596509583661220827334811807036762762868717$ 3078386032862664941787152719793655681013054394917306894307411392 $3800046207479053341773213890632297,1\}\}$.

(34) $5^{278}+1=\{\{2,1\},\{13,1\},\{557,1\}$, $\{1421780856088393569434871474512274262838939257349709384666871351$ 22319737041156689018188194968991282673415275258596318868770157807 $61896727104627241166150753931528110248588493803562075537618493,1\}\}$.

(35) $5^{288}+1=\{\{2,1\},\{193,1\},\{577,1\},\{641,1\},\{597889,1\}$, $\{75068993,1\},\{241931001601,1\},\{5207826497153857,1\}$, $\{249063976463696053897541171205805194685595629840418536549159091$ 4367246690460291622641850079223807032451616325315875604043659018 $33516758165042015104970817,1\}\}$. 
(36) $5^{292}+1=\{\{2,1\},\{313,1\},\{1753,1\},\{38907833,1\}$, $\{294339110898388933030666438674087732674377365128415431429470554$ 5331240383125935010573158144781268892750864406933733188794719258030249 $2291089490612485533127588619024028737968920842242864491249,1\}\}$.

(37) $5^{296}+1=\{\{2,1\},\{17,1\},\{593,1\},\{11489,1\},\{177601,1\}$, $\{190923785574671131653743190449789430957207593248212541787481096$ 5332328967741176419199814392853404493381153581876550320297624403508261 $3901266190175925748583706061771056186858601418196305366510257,1\}\}$.

(38) $5^{300}+1=\{\{2,1\},\{241,1\},\{313,1\},\{1201,1\},\{4001,1\}$, $\{132001,1\},\{390001,1\},\{776401,1\},\{632133361,1\}$, $\{268015942008645655782972335083605933065215410676092420009789340$ 8368820122825582473684672940279559807505636252923705499432233680678453 $1921675957836683199646871815576265626401,1\}\}$.

(39) $5^{306}+1=\{\{2,1\},\{13,1\},\{37,1\},\{601,1\},\{613,1\},\{1973,1\},\{6733,1\}$, $\{20129,1\},\{45289,1\},\{4613053,1\},\{6597973,1\}$, $\{19724557,1\},\{34288117,1\},\{455983453,1\},\{2194449013,1\},\{12447002677,1\}$, $\{69706205883723269045247554427866931903503113700425685700893703637833$ $96575220805069555573287953951783497010815989993679571729768142853,1\}\}$.

(40) $5^{308}+1=\{\{2,1\},\{313,1\},\{617,1\}$, $\{496480091466470356616586471672297140921286022641270883635570153$ 9193596217204939536429817927496447289156215099970043755066010993644391 7435737418133661475547675715466522403922044612552361363606186324603385 $2056953,1\}\}$.

(41) $5^{312}+1=\{\{2,1\},\{17,1\},\{1249,1\},\{1873,1\},\{11489,1\},\{247161617,1\},\{825256433,1\}$, $\{2220784177,1\},\{152587500001,1\},\{31308249137777,1\}$, $\{606074415387062678412559548607895202366463629864617011376740987$ 1229460244359687975532732870984667465498881690824336109844863077147895 $5444613274070471745777,1\}\}$.

(42) $5^{317}+1=\{\{2,1\},\{3,1\},\{4084229,1\}$, $\{152837540196438489848172875512405615842958310737691522401156757$ 0071897952399828592532114824295851067914508479856351515268177612770134 3978523607986505515170434340632108442646838495540184527007012174263493 $875473947749,1\}\}$.

(43) $5^{322}+1=\{\{2,1\},\{13,1\},\{1289,1\},\{424397,1\},\{234750601,1\},\{4195885464702037,1\}$, $\{835434658119628075220035657635966169042520482802263721821738480$ 3147739159410039153952222497500197147170505485125617423002057750608437 $3841986609334168774188284329748227979306119005808207269281,1\}\}$.

(44) $5^{326}+1=\{\{2,1\},\{13,1\},\{653,1\},\{2609,1\},\{189895001,1\}$, $\{869655550487180266344072498631650735681817697649453217338387875$ 0147004986203699135669385279111703381040686778239182004111154628467501 7625378824572082152572328861600485384920520491675698974411442827859200 $804531413,1\}\}$.

(45) $5^{330}+1=\{\{2,1\},\{13,1\},\{41,1\},\{89,1\},\{601,1\},\{1321,1\},\{1453,1\},\{2281,1\},\{9161,1\}$, $\{245521,1\},\{1458601,1\},\{69566521,1\},\{4411608961,1\},\{1030330938209,1\}$, $\{2802204291103029121,1\}$, $\{630005246595051274112917064925622507047391294321702273077957398$ 5265187306363726979961697471339384054138187777745310141217039742942676 $96079995983808277,1\}\}$.

(46) $5^{336}+1=\{\{2,1\},\{97,1\},\{673,1\},\{2593,1\},\{2689,1\},\{271489,1\},\{1149569,1\}$, $\{10922689,1\},\{29423041,1\},\{8906835789071809,1\},\{465314420407473281,1\}$, $\{188771740273710528624970810669294269237809741061817982897827641$ 
8453840306134730899905253198740159316022300870630717951772044195838210 $4072809394530745916100932702209,1\}\}$.

(47) $5^{338}+1=\{\{2,1\},\{13,3\},\{53,1\},\{677,1\}$,

$\{83181652304609,1\},\{349709778373997142089,1\}$,

$\{389405028944403743453918702180846517224301907787264264988554665$

5413928039417819520003167851100985417051859162163917826339258281156224

$5195804867673506545118774616078414982196530165997828063541609,1\}\}$.

(48) $5^{352}+1=\{\{2,1\},\{641,1\},\{1409,1\},\{75068993,1\},\{369059329,1\},\{241931001601,1\}$, $\{900314420999410329315082241356645899367693864865800214773511137$

3919082646867035456459477797373670741131631970209247976775640095404501 1363826100119510369838029228200065838125423529460804958222291076451922 $315543041,1\}\}$.

(49) $5^{356}+1=\{\{2,1\},\{313,1\},\{2137,1\}$,

$\{509263661549142609212706441504220792427543522307935982744320749$

6516012658449622143592103090059196660629428367883381977220096946028915 3607910429162969564423205560916789479383085750456285834270296848549416 $0218383095858740643423798517094256332073,1\}\}$.

(50) $5^{370}+1=\{\{2,1\},\{13,1\},\{41,1\},\{1481,1\},\{9161,1\},\{9769,1\}$,

$\{40849,1\},\{5121318001,1\}$,

$\{140680264279175963763498571557655236639189495448342387634086927$

1467718938809642591718369822367953423220834893516601602815291619922523

1447892833251624707882926691941456602256327851213484279405816911610123

$3210589802597659227215115841,1\}\}$.

(51) $5^{378}+1=\{\{2,1\},\{13,1\},\{37,1\},\{601,1\},\{757,1\},\{2521,1\},\{6597973,1\},\{234750601,1\}$, $\{4661402165281,1\},\{24587411156281,1\},\{147053007410401,1\}$,

$\{563926930546876101445055662754938357880160974326483141239232493$

3098131513014052219829546764566598755890764247289464689416339059653904 $1944631731046996358597276639862148988398519485771155181454736853,1\}\}$

(52) $5^{386}+1=\{\{2,1\},\{13,1\},\{773,1\},\{157489,1\},\{3357768242667569,1\}$,

$\{596991144619439286726859673687548389452047322110385930651836986$

1175244615885786906665854448799933864738938301552170168157493649521911

8788814386762099204247263928693864522395999146761726389395552928346300 $120464137435735000444664733168135230885357,1\}\}$.

(53) $5^{398}+1=\{\{2,1\},\{13,1\},\{797,1\}$,

$\{747532461123312088180297390847669500544391312044935984072534107005967$ 8309232093359449246663984199588665297833235314096205715348709569250681 4444900118779084876024246023946159689961046971782159136971515638383456 $08339313329143092305466299123888417438642224710633865528936931933,1\}\}$.

(54) $5^{428}+1=\{\{2,1\},\{313,1\},\{857,1\}$,

$\{881695186297,1\},\{1151785652724457273,1\}$,

$\{264799137712800759892621927038377156545797963073050688447010502$

6046758178350553814266771291431363200876556547539139076665205314920178 9996735679520958750537624008367561596991556958914602072607397544557013 $9750312396691966292820564141862400582061841299849206941363753,1\}\}$

(55) $5^{438}+1=\{\{2,1\},\{13,1\},\{293,1\},\{601,1\},\{877,1\},\{27133517,1\},\{84128413,1\}$,

$\{7032466681,1\},\{1278677279155561,1\},\{4725897742147117,1\}$,

$\{361697183870466527423525410190221612230419162055197629673670624$

2834410945903249376197101652635885900411054053670591576887957052525114

7490622791564792508023691413968380572743686407208745033152922272547695

$07355961432818549154008732733425114893,1\}\}$.

(56) $5^{450}+1=\{\{2,1\},\{13,1\},\{37,1\},\{41,1\},\{601,1\},\{1801,1\},\{2281,1\},\{9161,1\},\{14401,1\}$, 
$\{239201,1\},\{2516401,1\},\{5115601,1\},\{6597973,1\}$, $\{20478961,1\},\{69566521,1\},\{424256201,1\}$, $\{6794091374761,1\},\{25535754811081,1\},\{89620825374601,1\}$, $\{140222774541514676273575657072181995360342973930145870156030273$ 8592232351091447716987217069436484495284144032091609290478591508671759 2309851155850214835539604541264775253449726649894654067021910975343180 $1,1\}\}$.

(57) $5^{468}+1=\{\{2,1\},\{73,1\},\{313,1\},\{937,1\},\{51169,1\},\{390001,1\},\{543097,1\}$, $\{1503418321,1\},\{537181587281,1\}$, $\{350085589632986144124855155827661025192605691074693846093110667$ 3732948156939834005933056450513972166133986154897384010543483929764798 7742461535339868189060527068498663614404748967790081024648111285186566 8235510131305466545518265411790553140011877019458856340620421744527083 $3232978057,1\}\}$.

(58) $5^{476}+1=\{\{2,1\},\{137,1\},\{313,1\},\{953,1\},\{623017,1\}$, $\{938894988049,1\},\{1361753880209,1\},\{4959636586609,1\}$, $\{59509429687890001,1\},\{121008044805780049,1\}$, $\{220429053785324125717395844149771428966901833530768504710638771$ 3820457972242710179195505965797427440815747358294323352075351892184694 6334374002228589445832506727772294706984093361616132028556574933066005 $9736386168226475095963262131532566985710767033,1\}\}$.

(59) $5^{488}+1=\{\{2,1\},\{17,1\},\{977,1\},\{11489,1\}$, $\{46455649,1\},\{13438797761,1\},\{188732040042673,1\}$, $\{278267074028956475652980480030019198615537054871783247368584274$ 1346800899470085677056403681167873563332544060370682523398109759834149 0619820531900046828060902531325887480916478505197627467089238285442674 4359313360173417675580154580406271632389336212132098408775902766340942 $4757552702616003374260544929,1\}\}$.

(60) $5^{506}+1=\{\{2,1\},\{13,1\},\{89,1\},\{1013,1\},\{2453089,1\},\{1030330938209,1\}$, $\{805673999133305440716447735280032665499798706970849271569420304$ 1339488499303316022895147036094079333059901935968065565052360895220614 4638308837972583929612929460800791989642493473012635033847275665370792 5161662256589794256430846915868891511700529877446678168226035972274075 $08412581619044787195310807186696888835161190995628226293,1\}\}$.

(61) $5^{516}+1=\{\{2,1\},\{313,1\},\{1033,1\},\{4129,1\},\{18233,1\},\{390001,1\},\{3084493201,1\}$, $\{30185745097,1\},\{19511773794697,1\},\{45245904640297,1\},\{52396512454609,1\}$, $\{570052323619818830553659046643745387534547577907887650776887483$ 7779586769813557816189995160817243502393658871307615023434696074830226 4133693780145484675690724396733046572964740416228460874570348401745205 2822479940026600956558468104516471127390141121204768905092660633385310 $19686553,1\}\}$.

(62) $5^{522}+1=\{\{2,1\},\{13,1\},\{37,1\},\{601,1\},\{2089,1\}$, $\{31321,1\},\{382801,1\},\{6597973,1\},\{506670253,1\}$, $\{150456049677438917687187997004381249485867566704109650993869114$ 4676208555440398807594026590320501149837298067403736968456411083671475 7815162095922905013176049274011233964988977612521818011263591521388021 2024383537459305520283860535685242419619546302351140457412722294430269 $7877891747293751879590593630087587907493344440898447502693,1\}\}$.

(63) $5^{540}+1=\{\{2,1\},\{73,1\},\{241,1\},\{313,1\},\{2161,1\},\{8641,1\},\{73009,1\},\{390001,1\}$, $\{440641,1\},\{543097,1\},\{1853281,1\},\{3314953,1\},\{632133361,1\}$, $\{1503418321,1\},\{36280398313,1\},\{356646293281,1\}$, 
$\{50735307557193841,1\},\{23320317172851318360001,1\}$, $\{221821274240305797650139799907482950938580901848648426690221542$ 4930563212496300561334787899238530856720220040234593477239855558206468 3481245902397416917464143405011569617574279148121153181777391138521886 $58530135276851598629121685266588528146085030961,1\}\}$.

(64) $5^{546}+1=\{\{2,1\},\{13,2\},\{53,1\},\{157,1\},\{601,1\},\{1093,1\},\{2521,1\},\{8684521,1\}$, $\{234750601,1\},\{3640732369,1\},\{4056854881,1\},\{24587411156281,1\}$, $\{83181652304609,1\},\{667929705480493,1\},\{1636546425044077,1\}$, $\{141437955091157453,1\},\{9202941751066205689,1\},\{199852066328056058281,1\}$, $\{532268954040280358204574309336592613206940512768107642956497304$ 3035116436582420564370745254646578301207725133470563342680116166782387 4665372394272256832475752429800743681476117617281306619668838803879318 $400955482153557,1\}\}$.

(65) $5^{548}+1=\{\{2,1\},\{313,1\},\{1097,1\},\{591841,1\}$, $\{2670400296640306866605025933262480961124941182600601999918557590$ 7030359845065103435706725656905136427961423698846988942443608318113218 0822287432711801164268286906240308909276231842805013779405165361402496 1140403447988875086312284114156543707076708267900482848586696558632174 0421111698643522620272314003833564411203368243029049063446133505430014 $80302794963905793413966391513,1\}\}$.

(66) $5^{576}+1=\{\{2,1\},\{769,1\},\{1153,1\},\{3457,1\},\{740737,1\},\{1078601223169,1\}$, $\{3666499598977,1\},\{4134866393089,1\},\{9258693158017,1\}$, $\{588098015858334994923391953402349605574461466738756510696185444$ 8082706308647165968532379508679607599930838547990496969633588552241926 5722460029197237453024243273517352855801986953301127349286255976897608 9694574877537682449344905441680411821799341926002277380677553767183177 $3484044687116092571148101059083897097349115959649759100714198529,1\}\}$

(67) $5^{596}+1=\{\{2,1\},\{313,1\},\{1193,1\},\{31908060963841,1\}$, $\{161810758709030342748115336108890708166254282763015323842871164$ 2598640733741620285921978230013825246627192481537158200312726021407616 7597072712614372766086415532082112486444449772293061072705395712591003 5300393158449201787570998731984763032972994095404384657266121745510224 2658428369893597178161262205255396603289158769847084427257422440875299 $5154247168265542715903546685579790186985810141058096377,1\}\}$

(68) $5^{606}+1=\{\{2,1\},\{13,1\},\{601,1\},\{809,1\},\{1213,1\},\{3637,1\}$, $\{145441,1\},\{5728721,1\},\{3375268261753121,1\}$, $\{240087397921639578038881160320918428680654141260655896218139218$ 8692752196362886867882391438126981150996004784161756606069163286506740 2068893325545185868295434808435695601142918943001001186575062583850839 8900826129701387838620946895802172070960210989110924521609609566603456 5759382708913949908308524914329931554311165707359077832526986552923880 $7144405074449513063969237995634565731449,1\}\}$

(69) $5^{608}+1=\{\{2,1\},\{641,1\},\{1217,1\},\{69313,1\},\{75068993,1\},\{241931001601,1\}$, $\{479310809031887789380937713661303605693080937797915058960747213$ 9283380069971163948532551175799348917248113394169612506217066341644882 3285161017932596174057129880909071983844213558732122123159695460270101 6604978136405740096030482893883886257844999574327775760343909715196674 2017926455647548302948574549862056753442294621778469135910448676891691 $3339947482256220020370956889749372161945724318484481,1\}\}$

(70) $5^{618}+1=\{\{2,1\},\{13,1\},\{601,1\},\{1237,1\},\{788569,1\}$, $\{139210681,1\},\{968889277,1\},\{5223725024289757,1\}$, 
$\{856009224424023468974654018725947921712360581815387420089723734$ 5042490780684041517176526248043296414460001733341179897338353307839389 30080410734064411686062562891628255324036451947343886885512944621716471 8487744327840535468312166361147353197316796265059132324197593425396835 7477331668855237049808926278149396024234240565885298577563047791128799 $2453984242443816704194821343918429330229213,1\}\}$

(71) $5^{638}+1=\{\{2,1\},\{13,1\},\{89,1\},\{1277,1\},\{8933,1\},\{35729,1\},\{1030330938209,1\}$, $\{902222344372805779483701230276718510604516841495945057037940158$ 3608594928490668928234559968753121886607779270931261225654142124651207 1541116184628153900894918768721465931341979556281315538715617345375128 7067302566667221279099105580043019636384630976409751043120588752448019 6071588816454503602655000358188299220190171096138728297497212730724777 9055577508042125392422150199326381342435727654318824139088450007915122 $971409,1\}\}$

(72) $5^{686}+1=\{\{2,1\},\{13,1\},\{197,1\},\{1373,1\},\{41161,1\},\{234750601,1\}$, $\{26557257300533,1\},\{164204573839769,1\},\{74535675530760254357,1\}$, $\{141022227357142943005379913280349730268812456931066871663970999405412$ 3304754050455206253150526334291815463639765590129220104146423974152815 6232732687505749414882467676601815386773624396089242184503192197513645 8802843448643750247689104610741246253239345223827657299465430122029617 6266807385869941916326517087881556064355541221824129792694734226065050 $4068151031530858772242889627379508268823696135506468469983008249,1\}\}$

(73) $5^{716}+1=\{\{2,1\},\{313,1\},\{1433,1\}$, $\{323372125309573944624998797514232440959342196148782269394595415$ 3643170474188317966874650222876912359422062597391825969895677639638689 6242358707182345798786730119969511970890423733998942571406701112768862 9706374703591852185021174908174509089030600705264231752074065082088411 4129568870436529038393150914709534011556637604345136332379757274491111 8717693526892925275940474515275088494445950714563710908464756824390738 0424231501053245747407223162597335771846453161944857918675397509723340 $159041347097,1\}\}$

(74) $5^{746}+1=\{\{2,1\},\{13,1\},\{1493,1\},\{3701716157,1\}$,

$\{188012603975665635942758044355549405822374134083382683914211315$

75202435833463158577232073370648978021575445337839078625556939467600367 0986272235906671410661497883641598339472468076661943320893634610099742 9330229415161809590915391310932479259476702911686391703756173656906194 7681901027641400262842866060019767395479702913963856911320925602993970 5996498737979058662736908964469533532912770391937519173059545436321042 5630392573457107317823829015286753166874842336477273446526497785221675 $4637445120260142623473201,1\}\}$

(75) $5^{776}+1=\{\{2,1\},\{17,1\},\{1553,1\},\{4657,1\},\{11489,1\}$, $\{890603760576790734824675462256693669140888207812323548893643185$ 5548180321436422179622219320122431785933006272342820317179863118052254 8349076324626155315692326548870285646232600933774328982265417243864068 2255401889845274832892813021603001460487200988924991322470038454035986 0227721086451916987635021572601636420817421249457832697359405916722179 4160255191715778331782581183450157888985760955351753401871343110093919 2615792030474485771917720644059498549798703553665510162719672965869293 $11796056067415850458574124194430256186992742081,1\}\}$

(76) $5^{806}+1=\{\{2,1\},\{13,2\},\{53,1\},\{1613,1\},\{6449,1\},\{83181652304609,1\}$, $\{151173837543542328734054849876953576008873188692430175359118099$ 
7484263588048627320779544402125280388535964395432393687386573220771641 8977961424319517441747420923495158956612663587835168090379139718223988 9798855133291381975011102472191113710848220693526366967973118133698559 1744858178110767918566748351109278545850605202905130057714316552598677 0172858400594766282867322118065728492035533437705643787819339097372575 5825488627814615882674729447444215511834068582317583369502339648129490 $57910471174294785553647570783118842211799636850374074173,1\}\}$

(77) $5^{818}+1=\{\{2,1\},\{13,1\},\{1637,1\},\{782009,1\},\{2161157,1\}$, $\{795322831968988902432201834114320089852642548693195853170960260423956$ 2504475890789862057240928908533238080196321260158418670769939742471144 9579211511063902061120375203670213470124890870862837402303826093223891 6254416856031368083480669345634550256405092068628798830955025808172616 7710251051071897798983050994022145169434205841387350009778107412587341 4080060991364213507796957526574451952111440618510412558217652106384567 9796567242786688287074178165387958029829713119435839842411237136197322 $228690980348577671202568901294001046578497862618410214352992047921,1\}\}$

(78) $5^{846}+1=\{\{2,1\},\{13,1\},\{37,1\},\{601,1\},\{1693,1\},\{5077,1\},\{12409,1\},\{412849,1\}$, $\{6597973,1\},\{329573417220613,1\},\{351416125984969,1\}$, $\{254754032198578321,1\}$, $\{4300079834361938482890644545821301642929588583323544886323104982$ 4185436271342097003585782822212311296417957043397694650064672867702940 9286424720134237812523489811486691010159336445866763858287544486368369 8599603159707018260717996757243164715878655924957379822370301357967470 5991341102061812916198872151015898379011638638344924474936041656216081 2357867751322931992298028132526206809723950122979398321225888429696115 3342896059934432494220767238380485815951997796655038647060254716829373 $351703885345791579904693865353973,1\}\}$

(79) $5^{848}+1=\{\{2,1\},\{1697,1\},\{2593,1\},\{29423041,1\},\{53373121,1\},\{152929478928472033,1\}$, $\{252085553284359656246560557228561602333685812338980955536859620$ 9494645453334192310369345388566177658542201571159409377605355901338901 1927476131767885390166362599200630392741559057815265099017763554979828 0496283875635945134215708377737148460914214982095752291563790804449233 0745790943413733804817391348416208069904157365882612761217715288787785 1167425376734402086366730638087469094428315084561225653509046614201398 9603164407166721330307289196645183793394367082375195519192240201493792 0556964132717889618204494129308194562308993472031738542318554077575328 $1,1\}\}$

(80) $5^{866}+1=\{\{2,1\},\{13,1\},\{1733,1\},\{5197,1\},\{17321,1\},\{7748969,1\},\{136590198133,1\}$, $\{473437557538640117387606541254486417815890033163458845689133947$ 4469927497584443491499170051227963045459877947111319977881200929694636 6276325048882928678147907594461783655218554161878457584645748994792833 7127919158878183495336724150291551088313964043115927575480235619142786 6339938715813363558854229565369932356024882828184632793257521468438225 8030124354775803390632716635281684561643124712479732671068906694795503 1655490247938937838804598644320017259155192908088150694694251083313513 9701055137505887948011526657924668429655867317678043028650818436690467 $2495708691148838069453,1\}\}$

(81) $5^{888}+1=\{\{2,1\},\{17,1\},\{593,1\},\{1777,1\},\{11489,1\}$, $\{177601,1\},\{152587500001,1\},\{814954734175776096337,1\}$, $\{533043597598496948536122815402502415693996385980806925691980171$ 5733996704164077253517330837072799654773750462281476195760272093642920 
7453313828835904328984652167905301178380698932305825986951016368200518 8597656910683996205957480509763126890073232850503321314087732304511182 6506865231955226692748515877977991482086976565708347340763621792936958 1776502065075910177074869788596261048549264824484352888850451752262311 6485135783098409504475972211191307967822553343919541164853514754413416 6109569004071521972462900999514324440792135785529139383558210902046068 $7879840186619383793,1\}\}$

(82) $5^{938}+1=\{\{2,1\},\{13,1\},\{1877,1\},\{20369,1\},\{75041,1\},\{234750601,1\}$, $\{22602365853058769,1\},\{29502402733290397,1\}$, $\{368587094674615655859264709276274622069522630475983892545781056$ 1924758536317588923958376972719952155073047946622462985206145067017598 7731062357223693907136700169652055372306650502804101388837276483422148 3262227650337372332792716073329635878773409362124529260693794256313467 8550233649802214320830519525771982901024440494399266700008530544350561 4123617160896243936741659981042682797899490713745483717086349922318462 4309397052039042851802305377070675526292495169517352291217672669988245 5468708255041410612977452672195596138507159606401680538456147191573758 $892963115332905515483442337594938666144074613129,1\}\}$

(83) $5^{956}+1=\{\{2,1\},\{313,1\},\{1913,1\}$, $\{137099175184365798808422924004587140419347736015832870346356958$ 4109724790704675156319930836444036525519400719636968696550723945374050 2767752286923328031793906937567332307535893890567325962001784222092866 5113118535645953223658776783272898072221503868134067995517148656446621 3830266632156503789874393209031248417430475643652423639451202619423064 7535812520068712160733700607366285078927959188173207058033061639946060 0369813988434149775578469010967281146874871164782383685463033964137058 1787761820797286355883758797619720249564453033916593147940824925867851 2242669058791223442821241739219141515440856815407460629948601116722879 $2804076733732992935094668892572844517977,1\}\}$

(84) $5^{966}+1=\{\{2,1\},\{13,1\},\{277,1\},\{601,1\},\{1289,1\},\{1933,1\},\{2521,1\},\{424397,1\}$, $\{70942489,1\},\{234750601,1\},\{22600337281,1\}$, $\{24587411156281,1\},\{66988220431117,1\},\{4195885464702037,1\}$, $\{534199839612725905931780358867174243538398122527566766199044573$ 5402491283434418825145037419945710101369939578353014704178683613830193 4795268839863214416956116641138219781506904603947827662949980150913539 0672648247968075801687878338496599769241587265913779834602527998339549 3465119824762819498510188776933461665790583688685115016105337952650948 3773311879453859774779506705926924715007592466908526213360375524558113 0668345569857614899870973687472808837830149914478282326773112483774001 7158291835065155892222013536544447831897953501993632332717274581049946 $8897164394021168096652473403597,1\}\}$

(85) $5^{996}+1=\{\{2,1\},\{313,1\},\{1993,1\},\{390001,1\},\{2058554713,1\},\{2183732799044857,1\}$, $\{6826766595013505263246558239138585575484677654663588302901905792$ 4568155027142495813943632419925527234205737940648183189786007613605259 5713947521775199388308945316610105137418360929496911130170551375519885 4213229518685883879875275263922209846845884847257563438841769550829056 5728964124946861798798817068719278744175596932913268446958388428485087 9340169177518415464382606863844708363671241694542462385513879874015116 3597962565647526292474695883006641101477555027354119041946042442732055 6195186099134045130730316450151730828647780816773776546373710054531219 8082063260382406610607120239181600561486547298290321635245839498440759 
$0680736091667157606831548641638126777,1\}\}$

(86) $5^{998}+1=\{\{2,1\},\{13,1\},\{1997,1\},\{1413169,1\}$,

$\{361858067533,1\},\{6208302687649,1\},\{525570436885493,1\}$,

$\{430900245685035560839348419168240375335676137613344707098477473$

8249293564739160571890831604703541560629348320397640146436172158708202

2358964470576505865103939069297416789487402712420187759376332438696280

0673604733871779012947430667566900104852150292492377775819308882283459

6798040692435096869726145184407689295308765810915764110729932523910239

5358144607980012176448016507792445694777992035828170708652937224269289

3504933793265663043928344535497107600461280044640492043654416791501473

9378338212242737917329341478314529217280024955618702093398464043420485

6687279193673304152486403126744841065541425418530291366899965067616380 $5677316244052584312188797,1\}\}$

(87) $5^{1008}+1=\{\{2,1\},\{97,1\},\{673,1\},\{2017,1\},\{2593,1\},\{2689,1\},\{271489,1\},\{1149569,1\}$, $\{10922689,1\},\{29423041,1\},\{125837710159393,1\},\{8906835789071809,1\}$, $\{176607726594127565522376534268082605975813789484828001935299504$ 3374860042092578592102559212083387135867354581720136424739017982336711 8458224398140621713415851624622504892794280151187378637371242629991837 6780217665006728738224937837899833837580891445333127577033519377853240 5295990256829877275100515066623292198066609943151310684929688946480023 0598727974838172751048492594072254010822324542340855210496719746573441 9823104897563231576419609591114142849365707415679064713166020942053268 0001638586051392903650326409103104402805222933686676924447922799814471 2016530512269744543855526129505799931333621881415364854411452462673477 $24445068609,1\}\}$

(88) $5^{1026}+1=\{\{2,1\},\{13,1\},\{37,1\},\{601,1\},\{2053,1\},\{20521,1\},\{22573,1\},\{6597973,1\}$, $\{39262969,1\},\{50164561,1\},\{53590717,1\},\{4885168129,1\}$,

$\{16610859973,1\},\{197991079813,1\}$, $\{2108505761893,1\},\{2864226125209369,1\},\{90492378184051683013,1\}$, $\{413624777408487154693994160136794511500359567313163333275428933$ 7330851372120282209951433503542297486701594027806373096115102786536506 5556155803581419347186171377225474612287969786313063897696319593969930 1907047024843038877601321123278227487643635417569852307570463070911662 7898999577631694599963618040254782631717938777615719380571937752471752 8796321197076829272050241195845923628375680704799831832102356330287586 3069587089186498747878105393357685841378069622186229995601371033776304 7502212670483538896607886917425139786621639708225745649557269699729599 $64337857166932395242018338385790589213,1\}\}$

(89) $5^{1056}+1=\{\{2,1\},\{193,1\},\{641,1\},\{1409,1\},\{2113,1\},\{6337,1\},\{27457,1\},\{325249,1\}$, $\{18841153,1\},\{75068993,1\},\{369059329,1\},\{241931001601,1\}$,

$\{5207826497153857,1\}$,

\{ 472393277961844420483517026121640537173718336266522965835949891 0238825770159449020271279999575316449082698231501956554286743070880726 1688065094716534852221427561115328579673506065489049500158393997129920 4839829918240872703829721663772263352773881437531495477800364974137438 9145452067949377289642537507841539625473362488993070797848677398550816 0901600164521947722561988575999010498849645977531672235344379046059352 2889920682467939634535106668376566251286460764360073341171296728405975 2953601167297658454832455390580780563340020698681173748525208424739406 7688583829551995614644790884208651973944050445002678120182323940823429 $989632193763544039173800932893504270209,1\}\}$ 
(90) $5^{1076}+1=\{\{2,1\},\{313,1\},\{2153,1\},\{93375281,1\}$,

$\{981464014456781780989528670602116632801604737063001906146902749$ 6355282056090942170053035939621690516306170745374734133114212340461471 0946149741378977634976034464291834011110871218119232965215515662867873 4480999631903577025142512412594976134280582145883485352167112330805922 9385675717616665124253191085542153565738555625297788178020141506755358 3574998268088515059970938959269734031312822413905803590866126818923535 1811644511755393707976561516261096127997120262462467968804437672014484 4533085665569010603941313338568092415780844570198339891364607396154850 3648468570347986979195793694833521127941192373803106902620436980496581 5240276726140814828571502706932569718290902380801100475207748312130292 $825488008409148901202249250779245861276102857,1\}\}$

(91) $5^{1106}+1=\{\{2,1\},\{13,1\},\{317,1\},\{2213,1\},\{41081,1\}$, $\{72997,1\},\{75209,1\},\{234750601,1\},\{10390269663517,1\}$, $\{114646659906363022880159309631671040141042318631975395199181720$ 2356547189997029376963013079096852230362685404152383540560955225834344 1970375336722605253690338666210573170730803809887219124480040642830584 5691100823601602257423563208051807120354473776932021745620812543688541 4075626585815062128927117387757201409855569551441527443613838921689696 9554924779366433786463738002377080572026073465465523887067277809100767 7970246820965666067411872984458413576079364569630831067374569635192640 0781459724852717581272745675763809218633825440681561688990911716335355 1965028401437499077367648108320882259064318275652137855567807084243221 6702681939139733075635263940098473137971618374958251081141844252057317 $67391923134088475624360590317553121561,1\}\}$

(92) $5^{1118}+1=\{\{2,1\},\{13,2\},\{53,1\},\{173,1\},\{2237,1\},\{2028053,1\},\{16146157,1\}$, $\{210362881,1\},\{2171388367013,1\},\{83181652304609,1\}$, $\{325594874300625823665614491717103316803413413118550040095974429$ 1235368725098382966425511331272348430100953798782195506695651738079964 6711718461934776020194426797023996575532233551552704256127457660590510 3004187735076143200270906979671365463633055208277048242666878793441298 5018770533036819142090923980698876807209001667950890484978571575566926 0816618047889361357672856665932032035739490604509003383700527896522035 8020924794038185069858839569381380516985162601525929085866408207252868 9264980798733954773431979330803697876778593084113373885999136529667695 3405240916345983017157432115025811034270013530722880404492595686780618 8365781263156513230412630180821050728224705200261292868079825232162928 $0994004912512274316086694552477,1\}\}$

(93) $5^{1136}+1=\{\{2,1\},\{2273,1\},\{2593,1\},\{68161,1\},\{29423041,1\},\{2393066459969,1\}$, $\{189371538391364593000065428626609616606728279782962923926854028311223$ 0735742137672891256359810364264640479108676627534064586440131875952565 7110562812027352042087833619699369490659559597567692016243330039032053 0029315121223650374322130183894827837072974241421553884646719756047916 4496512458589026592576274575829726966957658801480105874156302417776041 1821779101359513901617617825023559144725631263888118741739399307161913 1624118837911205477629738872842860595587141974215677471635928906645930 9830521259560535107103491536371187881740299855596639426046373245342754 2035772660879017249732448779013505116768072126894686744680417771120514 3294063932489003659672676649481465634509046965523294779515827746045443 $0856125605934918253191500788179695074451016542682494075856698593,1\}\}$

(94) $5^{1146}+1=\{\{2,1\},\{13,1\},\{601,1\},\{2293,1\},\{1053557,1\},\{5412888049,1\}$, 
$\{206218283317,1\},\{146292198125317,1\},\{234499916148809,1\}$, $\{723760684383988294606731559072930448058062928347250444054978446$ 7752400341093105207784535613365132297692674890438475072932284755870922 5379051920868034720422100607063781412071924978396434566531826884124939 5064315626923263037742120596622161628235382869805655748264019642091054 0331009031778728444042916265616260406125890193184671349503244320187455 7135167498355640062096016187990941328593828248301416030354210708014119 7932577645539914621961049032119679100100216646094174028656754007385450 8520136579116496454156533246130511550235868498081280261973335744084119 4651101549440461764538939110764056425030176769747752655570140966334751 6457814676769955833754204385450516883651074164016174647112155916042521 $732953363634419050092275682245461728266477249,1\}\}$

(95) $5^{1148}+1=\{\{2,1\},\{313,1\},\{2297,1\},\{2953,1\},\{737017,1\},\{5643569,1\},\{23579921,1\}$, $\{811755457273,1\},\{59509429687890001,1\}$, $\{130011421527587174042053118998687356397474274214523561150022322$ 4141581634716962709165281926995849035075426719108471714796420031179570 2386744272436693263000143855009361293164055564592413029099942265957976 5726829360373555308091984198021217519944660263043699891661313637127889 8891922881734031426108854809575762715719912102910143796290005601982990 2383693743843109497604614434068926776723734525710047675543675302923525 0114901938052375355768327421300122886925230502487318976159465991157946 8739738290426307968553944613124896886069455435341902778446709957293619 6457170971036487699246504639680465620502617629241162954137107144161869 6791361897136937647241907468121283945483857793296692229945521790878206 $1089177766960374001518527229979285034812092820125329,1\}\}$

(96) $5^{1166}+1=\{\{2,1\},\{13,1\},\{89,1\},\{2333,1\},\{1030330938209,1\}$,

$\{179378431261898945649166596314569100954763906609627684937958083$ 3863209859158418065812341990654679103083800110460322225031399278135901 0300970208834040783907662647584389424276384305997437787063633570811253 2283928566359683664159222255709273614733884137938329693020616182275084 5425306558197555086313649880273810255960602818611645769452425153311189 1878339340289650383078479552077987031255074351926065478046176380203628 4591450110940948188118354350172892931472877246532440859963953591259728 3778351345699015037934886521934932641198864600790627729879153790058181 4623551669731501257884692896503229932920058043157819785695285786031507 2405973693910361312803019794785582515800192664520276712928987431279432 0460455071963327318188674808789824788620657447708841748369393921859102 $1077481188547743210528881009228997,1\}\}$

(97) $5^{1178}+1=\{\{2,1\},\{13,1\},\{2357,1\},\{695437013,1\},\{4885168129,1\},\{2864226125209369,1\}$ $\{408495765793286142074897354283745125163347203645197827804753366$ 2491869851859606041895838187951276121979102693488519767073186093655198 5124775969683493262932723830547590578432429397794624958538514691278674 3352692359622202526094807609725876086842921584987439216094414460936897 1779865912173219629767827242462066309412974115365596362398997380680553 3014380644152274808211550568721974042194329747711093373461456438603353 9501283497248261151258265098284270013788622106490032504849391849390100 6086666173589306853949454630701069296341493539926084208394077981684090 8396426053055208113670725728532360940816221261981196002405992844188882 0736992225788195449748261108405843431213461972230676071493461525123364 9153532397347880150910323443743175820608558744590534247606067772655366 $0497090873993667580961,1\}\}$ 
(98) $5^{1188}+1=\{\{2,1\},\{73,1\},\{313,1\},\{2377,1\},\{73009,1\},\{390001,1\},\{543097,1\}$, $\{1853281,1\},\{3314953,1\},\{331660297,1\},\{1503418321,1\},\{36280398313,1\}$, $\{4352560631862697,1\},\{112835047682166217,1\},\{346866834441245977,1\}$, $\{748003465152963352127485818438857767619524836938715873533649800$ 4623682344956161485495236678010784380505806235765570511656566531408971 4388815178521948708444803618569264630465809530179145066084558716830330 5430487092219258013388357422213686150149204231840704959341818632663680 4410020894027249204055431956142779984490784776164381363798138674718067 2434697367090233241073903249457819990987274618176065861359418672139150 1450852591230474399384864204526980378343372851825432628789429287289182 4915202488494314508384966098067849721177724249881280434451252949214674 5800565269580364027831108392354848409291279348778352685168631719039758 9954953429351794872084557944185677759706258821911609306647877561762955 $1364997898831276891433,1\}\}$

(99) $5^{1196}+1=\{\{2,1\},\{313,1\},\{2393,1\},\{7177,1\},\{51169,1\},\{4099889,1\},\{537181587281,1\}$, $\{41383425174937,1\},\{150220315444217,1\}$, $\{7288953048306073,1\},\{129044008326199409,1\}$, $\{131161677173623738996438129908476011411205790413776421152759759$ 6005384677017730345802075703947584050957211196901495364640833236041780 1486655700195759513781581667265618742457465604719066281539360491419817 2051782007752409724518862691469439022012473367718703659695118860270453 5123112639215608162286091584041122726931092635782400342550142887138325 8183209070466778592394070176607208914795700127549586174332241711877711 5845410795092096260503690708084329602339944659269243416159648349798472 5314952106691211093020629226356320916383704094836809355159652018748154 4990780074239208326759827930283776296671473308981042711155056818483052 8111172892110213160482486552382469600148430201074841583545840868403031 $91972526055642004926643749948645910722684343151257,1\}\}$

(100) $5^{1208}+1=\{\{2,1\},\{17,1\},\{2417,1\},\{11489,1\}$, $\{10640829750743377,1\},\{17718805276155041,1\}$, $\{127443447430668730205889091330105122762626146088780506350565282$ 1279583685854753527124944822233684786070634581392854681048228875240624 2833188428721272925423813119292349235740611542593005118833155510117756 6508170298434855238797662013160349824019671640183293802799529691390905 0200432545770920012072271294288099032864212660192080488587004039381030 0531586917938996837991228379722890673936539954949747843611057096211344 4361743797637541126521779776997691996474869873776557321252002463342543 8734437441569977808090733494723102779216907646079284866193387134676640 9848223428458504873245185314420249272154681880974612052052530574404978 7922102543947321697923766727646761241711215807769509929950595193671767 1009029962440149247356160542923836007354293205252548715409483384916132 $01892963385984975433982383800689416446529,1\}\}$

(101) $5^{1218}+1=\{\{2,1\},\{13,1\},\{601,1\},\{2437,1\},\{2521,1\},\{382801,1\},\{234750601,1\}$, $\{506670253,1\},\{918848200649,1\},\{24587411156281,1\}$, $\{224350648831980416190188176664936920104575864809271676137598661$ 7068358769942242099667244181826045649320051253652991892050179079364492 4498463743875038012531661272291373986459146317716114731152525280702152 9465114926999530014243644712852715724632387207877709376665857768447036 9481585535481991214450874440286151381233522385188366914067326497869712 8348882383785807871703018530894423638354346523300295111294373209033938 1062625620570295796652865005348107146479127429274171986287993837242751 
8931195012824322428049807204505488001121481018949869965203676200385449 3871421294464553971850853589147447208090649983118852411136739825576390 7108375931204560533977308879002792104460155640842007607482090118932467 4388666980247022778037525615458620625008984556838325856746371224695309 $597850531659274256010358779609,1\}\}$

(102) $5^{1236}+1=\{\{2,1\},\{313,1\},\{2473,1\},\{390001,1\},\{875089,1\},\{143724625337,1\}$, $\{111295544672183565855896129724234764282302277864323988990592680$ 0657827722730965481161510105345239632977226342619750318148042057703234 0088145361108278292855986221460083695070332677953091565083913381993427 9017433584435966265206429546635100604895029561263080829815916178012432 8488769989905646787299306960234909905806320349767752462721582663787877 9472944536597522290448220910704756156897983601203335038248669509136649 6839555005924116062945779550524605408119519092741589284495965605139228 0077052586388178699232921498472096380382889879522089957584893509447857 3138222114041795272852558883088843707701492974430228116634832238193129 7670548268799957884861493061218269570336094970135710921729005516540197 8494957448993536100415158154613284706927824041267797860388181332042845 8865458259129786417300654602458524549494165031657145027811698507383022 $209,1\}\}$

(103) $5^{1238}+1=\{\{2,1\},\{13,1\},\{2477,1\},\{66853,1\},\{12096323689601,1\}$, $\{405687580707536691887496200203391158297150270626210432597491772$ 3898116684905969256495431893693592770345115468575576030461076195422485 0572715332439967396625465922763093217441161681873596147442922864549304 6596918248299150290982442357118144687523470898198138895759736921559919 8876837424709462102233473531138266950382497942970970580954119988489885 5431136579706326978360370965500825528912759984135203915420000756476976 5685155156961532046615175079998678267914490548652639943856017226097290 6444599170050747151429687002759936462176930237320672516057012305242266 7613067050154582138986140081692224169333269008623538805176070027602797 9262184987228167416062422813433741694464570983285092725881661551671174 2821874332088162839516303179426806027771026065475633161461100221971187 5744527991967546217529920447111161018373473457266410129948052226615384 $1969838521,1\}\}$ 


\section{Appendix F Factors of $7^{t}-1$}

(1) $7^{5}-1=\{\{2,1\},\{3,1\},\{2801,1\}\}$.

(2) $7^{7}-1=\{\{2,1\},\{329,1\},\{4733,1\}\}$

(3) $7^{9}-1=\{\{2,1\},\{3,3\},\{19,1\},\{37,1\}, 1063,1\}$.

(4) $7^{11}-1=\{\{2,1\},\{3,1\},\{1123,1\},\{293459,1\}\}$.

(5) $7^{15}-1=\{\{2,1\},\{3,2\}\{19,1\},\{31,1\},\{2801,1\},\{159871,1\}\}$.

(6) $7^{19}-1=\{\{2,1\},\{3,1\},\{419,1\},\{4534166740403,1\}\}$.

(7) $7^{21}-1=\{\{2,1\},\{3,2\},\{19,1\},\{29,1\},\{4733,1\},\{11898664849,1\}\}$.

(8) $7^{23}-1=\{\{2,1\},\{3,1\},\{47,1\},\{3083,1\},\{31479823396757,1\}\}$.

(9) $7^{25}-1=\{\{2,1\},\{3,1\},\{2551,1\},\{2801,1\},\{31280679788951,1\}\}$.

(10) $7^{27}-1=\{\{2,1\},\{3,4\},\{19,1\},\{37,1\},\{109,1\},\{811,1\},\{1063,1\},\{2377,1\},\{2583253,1\}\}$.

(11) $7^{29}-1=\{\{2,1\},\{3,1\},\{59,1\},\{127540261,1\},\{71316922984999,1\}\}$.

(12) $7^{31}-1=\{\{2,1\},\{3,1\},\{311,1\},\{21143,1\},\{3999088279399464409,1\}\}$.

(13) $7^{35}-1=\{\{2,1\},\{3,1\},\{29,1\},\{2801,1\},\{4733,1\},\{2127431041,1\},\{77192844961,1\}\}$.

(14) $7^{37}-1=\{\{2,1\},\{3,1\},\{223,1\},\{2887,1\},\{4805345109492315767981401,1\}\}$.

(15) $7^{39}-1=\{\{2,1\},\{3,2\},\{19,1\},\{486643,1\},\{7524739,1\},\{44975113,1\},\{16148168401,1\}\}$.

(16) $7^{41}-1=\{\{2,1\},\{3,1\},\{83,1\},\{20515909,1\},\{4362139336229068656094783,1\}\}$

(17) $7^{43}-1=\{\{2,1\},\{3,1\},\{166003607842448777,1\},\{2192537062271178641,1\}\}$.

(18) $7^{47}-1=\{\{2,1\},\{3,1\},\{13722816749522711,1\},\{63681511996418550459487,1\}\}$.

(19) $7^{51}-1=\{\{2,1\},\{3,2\},\{19,1\},\{103,1\},\{14009,1\},\{365773,1\},\{2316281689,1\}$,

$\{2767631689,1\},\{10879733611,1\}\}$.

(20) $7^{53}-1=\{\{2,1\},\{3,1\},\{8269,1\},\{319591,1\},\{38904276017035188056372051839841219,1\}\}$.

(21) $7^{57}-1=\{\{2,1\},\{3,2\},\{19,1\},\{103,1\},\{14009,1\},\{365773,1\},\{2316281689,1\}$,

$\{2767631689,1\},\{10879733611,1\}\}$

(22) $7^{59}-1=\{\{2,1\},\{3,1\},\{459257,1\},\{134927809,1\},\{550413361,1\}$,

$\{354639323684545612988577649,1\}\}$.

(23) $7^{61}-1=\{\{2,1\},\{3,1\},\{367,1\},\{4759,1\},\{177237331,1\}$,

$\{1914662449813727660680530326064591907,1\}\}$.

(24) $7^{63}-1=\{\{2,1\},\{3,3\},\{19,1\},\{29,1\},\{37,1\},\{1063,1\},\{4733,1\},\{11898664849,1\}$,

$\{2643999917660728787808396988849,1\}\}$

(25) $7^{65}-1=\{\{2,1\},\{3,1\},\{131,1\},\{2801,1\},\{157951,1\},\{787021,1\},\{16148168401,1\}$,

$\{4446437759531,1\},\{434502978835771,1\}\}$.

(26) $7^{69}-1=\{\{2,1\},\{3,2\},\{19,1\},\{47,1\},\{139,1\},\{3083,1\},\{402011881627,1\}$,

$\{31479823396757,1\},\{235169662395069356312233,1\}\}$.

(27) $7^{73}-1=\{\{2,1\},\{3,1\},\{439,1\},\{3675989,1\},\{359390389,1\},\{1958423494433591,1\}$,

$\{7222605228105536202757606969,1\}\}$.

(28) $7^{81}-1=\{\{2,1\},\{3,5\},\{19,1\},\{37,1\},\{109,1\},\{811,1\},\{1063,1\},\{1621,1\},\{2377,1\}$,

$\{3727,1\},\{2583253,1\},\{3368791,1\},\{70722308812401674174993533367023,1\}\}$.

(29) $7^{83}-1=\{\{2,1\},\{3,1\},\{167,1\},\{66733,1\},\{76066181,1\},\{7685542369,1\}$,

$\{62911130477521,1\},\{303567967057423,1\},\{18624275418445601,1\}\}$.

(30) $7^{85}-1=\{\{2,1\},\{3,1\},\{1531,1\},\{2801,1\},\{4931,1\},\{14009,1\},\{2767631689,1\}$,

$\{138497973518827432485604572537024087153816681041,1\}\}$.

(31) $7^{87}-1=\{\{2,1\},\{3,2\},\{19,1\},\{59,1\},\{127540261,1\},\{2576743207,1\}$,

$\{71316922984999,1\},\{196915704073465747,1\},\{358475907408445923469,1\}\}$.

(32) $7^{97}-1=\{\{2,1\},\{3,1\},\{389,1\},\{971,1\},\{4160924156784953821413999107825795646911807584$ $554735726748631757909547764679,1\}\}$.

(33) $7^{99}-1=\{\{2,1\},\{3,3\},\{19,1\},\{37,1\},\{199,1\},\{1063,1\},\{1123,1\},\{3631,1\},\{173647,1\}$, $\{293459,1\},\{1532917,1\},\{12323587,1\}$,

$\{14658652062814508164798530246267135510037033,1\}\}$. 
(34) $7^{101}-1=\{\{2,1\},\{3,1\},\{607,1\},\{809,1\},\{6263,1\},\{12269621530729809529800670968884$ $60031879516017670288348423584305043494594529,1\}\}$.

(35) $7^{103}-1=\{\{2,1\},\{3,1\},\{17923,1\},\{10316589882658148664018432412741304154103858145$ $253645339510927287724805842171756259,1\}\}$

(36) $7^{113}-1=\{\{2,1\},\{3,1\},\{227,1\},\{2300919443481866466139853110309106017717326808587$ $25360845463092718251327185092028815673428363,1\}\}$.

(37) $7^{115}-1=\{\{2,1\},\{3,1\},\{47,1\},\{1151,1\},\{2801,1\},\{3083,1\},\{188831,1\},\{1446701,1\}$, $\{723461377501,1\},\{31479823396757,1\}$, $\{880569873730854523701834429798456157718737303721,1\}\}$.

(38) $7^{117}-1=\{\{2,1\},\{3,3\},\{19,1\},\{37,1\},\{1063,1\},\{1873,1\},\{322921,1\},\{486643,1\}$, $\{2280097,1\},\{7524739,1\},\{44975113,1\},\{7687225261,1\},\{16148168401,1\}$, $\{661353842305791342187228684843715341,1\}\}$.

(39) $7^{119}-1=\{\{2,1\},\{3,1\},\{29,1\},\{4733,1\},\{12377,1\},\{14009,1\},\{34273,1\}$, $\{2767631689,1\},\{305213588009240737,1\}$, $\{8918549001667773167139703843417987904524866634456577513,1\}\}$.

(40) $7^{121}-1=\{\{2,1\},\{3,1\},\{1123,1\},\{1453,1\},\{65099,1\},\{293459,1\}$, $\{965927365520136304449338827049103841305646805820126141875147843$ $2947875933991840016519,1\}\}$.

(41) $7^{125}-1=\{\{2,1\},\{3,1\},\{251,1\},\{2551,1\},\{2801,1\},\{31280679788951,1\}$, $\{128863605961145736707054168690536807555069447341593411117481172$ $65345044452558757251,1\}\}$.

(42) $7^{127}-1=\{\{2,1\},\{3,1\},\{260461507,1\},\{13600544081983527726186578957448801595417$ $9502319831499442705083909311214909086172079670578359445251,1\}\}$

(43) $7^{129}-1=\{\{2,1\},\{3,2\},\{19,1\},\{10837,1\},\{63350976270733,1\},\{166003607842448777,1\}$, $\{26720288538918886221101121286198760763472341645886160933$ $7071699981802929,1\}\}$.

(44) $7^{131}-1=\{\{2,1\},\{3,1\}$, $\{850534611647968019495395416395428057706663923306826733025308197$ $74105141531698707146930307290253537320447270457,1\}\}$

(45) $7^{135}-1=\{\{2,1\},\{3,4\},\{19,1\},\{31,1\},\{37,1\}$, $\{109,1\},\{271,1\},\{811,1\},\{1063,1\},\{2377,1\},\{2801,1\}$, $\{159871,1\},\{185221,1\},\{2583253,1\},\{1527007411,1\},\{125096112091,1\}$, $\{175561876921802311,1\},\{797937984757981841530188510084780901,1\}\}$.

(46) $7^{137}-1=\{\{2,1\},\{3,1\},\{2741,1\},\{213721,1\},\{251533,1\}$, $\{679092646604348848436230554836412626083799829151411191949277186$ $09108870191730640104317939852348887577,1\}\}$.

(47) $7^{141}-1=\{\{2,1\},\{3,2\},\{19,1\},\{283,1\},\{5960917,1\}$, $\{249860742197241948192966954622328194519562899140309550615431262$ $021195649000642066629971358028858497510982063,1\}\}$.

(48) $7^{143}-1=\{\{2,1\},\{3,1\},\{1123,1\},\{293459,1\},\{16148168401,1\},\{528808508322667,1\}$, $\{418330546708532740480353541335711575735343912784641220943864002$ $340111144746922701991203,1\}\}$

(49) $7^{153}-1=\{\{2,1\},\{3,3\},\{19,1\},\{37,1\},\{103,1\},\{307,1\},\{613,1\},\{1063,1\},\{4591,1\}$, $\{14009,1\},\{44371,1\},\{365773,1\},\{2316281689,1\},\{2767631689,1\}$, $\{10879733611,1\},\{350379330748449481709242776279131$ $66980194052061378185812052293578251,1\}\}$.

(50) $7^{157}-1=\{\{2,1\},\{3,1\},\{4397,1\},\{5653,1\},\{1039945344901,1\}$, $\{308883753093321746997477641577980838910192868291755541765153082$ $11775675085491164240495874534303554491469173279661,1\}\}$.

(51) $7^{163}-1=\{\{2,1\},\{3,1\},\{653,1\},\{9781,1\},\{1260643,1\}$, $\{116665034851129307902624357139195905667066806190114833939064618$ 
$49438300220825949916393836219097792825097358411554362573921443,1\}\}$.

(52) $7^{175}-1=\{\{2,1\},\{3,1\},\{29,1\},\{701,1\},\{2551,1\},\{2801,1\},\{4733,1\}$,

$\{2127431041,1\},\{77192844961,1\},\{31280679788951,1\}$,

$\{368146726267000523206403038500165782925504879615058104729767146$

$296343146902066797513736881223461301,1\}\}$

(53) $7^{177}-1=\{\{2,1\},\{3,2\},\{19,1\},\{709,1\},\{89209,1\},\{459257,1\},\{134927809,1\}$, $\{550413361,1\}$,

$\{518114623020975074018002739309396360820240761273419101595672842$

$382758920962433239800530545492386878647395928477993621,1\}\}$

(54) $7^{181}-1=\{\{2,1\},\{3,1\},\{1811,1\},\{13873726021,1\},\{618805947190964867,1\}$, $\{983847868716989204697137458368555065405633130650561289091916359$ $7629083410743170729625030794757094333626527049443631027813,1\}\}$.

(55) $7^{185}-1=\{\{2,1\},\{3,1\},\{223,1\}$,

$\{1481,1\},\{2801,1\},\{2887,1\},\{6661,1\},\{219041,1\}$,

$\{942543691081369277773543497051844330874255595087425378964554439$ 488086830103129908995194

$71507227553132967611796458335693694020432329021,1\}\}$.

(56) $7^{189}-1=\{\{2,1\},\{3,4\},\{19,1\},\{29,1\},\{37,1\},\{109,1\},\{757,1\},\{811,1\}$,

$\{1063,1\},\{2377,1\},\{4733,1\},\{2583253,1\},\{11898664849,1\}$,

$\{651259195745827996896316544656062759285664227683642978154892763$

$46542233477985235950854607642865066771524511985220612893,1\}\}$

(57) $7^{191}-1=\{\{2,1\},\{3,1\},\{383,1\}$,

$\{112817278337559699810610328050113979428873512903449877053938675$

9511711123029010767110697767747142118787440023100116678238692690

$03853437734035902480275113415479,1\}\}$.

(58) $7^{193}-1=\{\{2,1\},\{3,1\},\{14669,1\}$,

$\{1443344374232042324865855590464004980021928503419390813461808380$

3650983887510085559095130207056419397592466215387544461142923560839401 $09479490884303451022801029,1\}\}$.

(59) $7^{203}-1=\{\{2,1\},\{3,1\},\{29,2\},\{59,1\},\{4733,1\}$,

$\{60089,1\},\{127540261,1\},\{71316922984999,1\}$,

$\{465941598913454377106883133376466664725890139166855163821838467$

8242557421304316103945154298716030603806966568704529767484855377352658

$621,1\}\}$

(60) $7^{205}-1=\{\{2,1\},\{3,1\},\{83,1\},\{2801,1\},\{20515909,1\}$,

$\{614419495366821529684317346059223964584194912846855305721440483$

1768597271836450638822525073986039974017465321311451759587334029901332

$082469935090737961932590783,1\}\}$.

(61) $7^{213}-1=\{\{2,1\},\{3,2\},\{19,1\},\{968299,1\},\{582053568181,1\},\{990643452963163,1\}$, $\{530843622319233468052258575559783397851526138568242511969574674$ 0445453578246760415887042361718235798297611223331504058841458542849352 $963113117069,1\}\}$.

(62) $7^{219}-1=\{\{2,1\},\{3,2\},\{19,1\},\{439,1\},\{877,1\}$, $\{3675989,1\},\{359390389,1\},\{1958423494433591,1\}$,

$\{350052570384438952909856290057637776638078892935130278004864659$ 6216462598019656046892083534715954735182304033668202205533353387492203 $27885461620797,1\}\}$.

(63) $7^{233}-1=\{\{2,1\},\{3,1\},\{467,1\},\{79227874003,1\}$, $\{364331892843505027296810945837058370824234171587813242775720155$ 3776250997296568609753935062992480523710205974489800023295868212705247 $21707945571681669954428431170606756683410356963001,1\}\}$. 
(64) $7^{237}-1=\{\{2,1\},\{3,2\},\{19,1\},\{2371,1\}$, $\{239484727876950684371642911666557190326884324967198043170126995$ 3766615830511447901288233785756775256772323788203171421130216403600807 $26956444197395407028365617023366854323555768814446622829244083,1\}\}$.

(65) $7^{239}-1=\{\{2,1\},\{3,1\},\{479,1\},\{871986721,1\},\{643384173113837,1\}$, $\{590152912740383546405391589472730230353037907605911579446756624$ 3450430617191658073783019793033763511377636151597787 $848749314554190951736701031949191094038770259580403873964979,1\}\}$.

(66) $7^{241}-1=\{\{2,1\},\{3,1\},\{1447,1\},\{28439,1\},\{33486078253526557,1\}$, $\{563935264197604425767391369648190015332073194595000645196540604$ 0846243915457765763636379382438528586672787890669697456610083951476498 $8288600174813891810007545918197353618197589621,1\}\}$.

(67) $7^{243}-1=\{\{2,1\},\{3,6\},\{19,1\},\{37,1\},\{109,1\},\{811,1\},\{1063,1\},\{1459,1\},\{1621,1\}$, $\{2377,1\},\{3727,1\},\{169129,1\},\{1382671,1\},\{2583253,1\},\{3368791,1\}$, $\{5563209703472098148970340309375524202401384108544444002078410534$ 889174201543076743361402681111820934427879395534500731216181 $8328810514486668104015552658777,1\}\}$.

(68) $7^{247}-1=\{\{2,1\},\{3,1\},\{419,1\},\{6917,1\}$, $\{16148168401,1\},\{22931541257,1\},\{4534166740403,1\},\{14975869588530371,1\}$, $\{125454655753267202797365863162986582917855663926667180423229018$ 4801205923867264403869350563252415166745855607627536006024738516449922 $74401607378216198999,1\}\}$.

(69) $7^{251}-1=\{\{2,1\},\{3,1\},\{503,1\},\{27611,1\},\{8895962998159,1\}$, $\{177669837175335119929176935701194838324252821819693597497081108$ 5363985997487004550001243529297221170285499484822210130977061003539415 $50906564040701778898732706050502407347416105293671115465331,1\}\}$.

(70) $7^{257}-1=\{\{2,1\},\{3,1\},\{1543,1\}$, $\{167370574641632502572384103567991159647198307969545382257664341$ 4741081747467696044279112555289002232175368458288635585784410406565303 9447077114058181760234621579650963117104467101671132607220834791233206 $22966778807,1\}\}$.

(71) $7^{261}-1=\{\{2,1\},\{3,3\},\{19,1\},\{37,1\},\{59,1\},\{523,1\},\{1063,1\},\{5743,1\}$, $\{127540261,1\},\{2576743207,1\},\{83226515419,1\},\{71316922984999,1\}$, $\{710074092185677,1\},\{196915704073465747,1\}$, $\{190746601014030887864608468381789807871176990791091071309234221$ $71376582328749617552725810181717044484513956643473221164545432614167,1\}\}$.

(72) $7^{265}-1=\{\{2,1\},\{3,1\},\{1061,1\},\{2801,1\},\{8269,1\}$, $\{319591,1\},\{3041671,1\},\{19069931,1\}$, $\{326807465819807698664810434867151304791310583012772124434804633$ 0001810484247056344701514282001017644502270562551132505922939451140708 $1604329366571993639434007656461042295654450080243087591264579,1\}\}$.

(73) $7^{269}-1=\{\{2,1\},\{3,1\},\{2153,1\}$, $\{166026527134191738669017246117715558254761946534264717237735920$ 2904266654414497656802586328365814688845871766391485540737993243972662 9980882705818126069237954655533417171726708563512476887150568030563589 $828993766083011007017,1\}\}$.

(74) $7^{273}-1=\{\{2,1\},\{3,2\},\{19,1\},\{29,1\},\{1093,1\},\{4733,1\},\{486643,1\},\{7524739,1\}$, $\{44975113,1\},\{7304123737,1\},\{11898664849,1\},\{16148168401,1\}$, $\{434230520613371097330409506950589908590603411661538993453297817$ 1022240582981318653732106062735677777169176587762334543654115724827134 $1177406015814087823821413193751043061,1\}\}$. 
(75) $7^{281}-1=\{\{2,1\},\{3,1\},\{563,1\},\{8431,1\},\{341614931501,1\},\{4420135738596254569,1\}$, $\{690300293879467318725802791181078584337726859227278494125038904$ 3225504690360237770469223932470619433141299551559599723644440976450348 $0503519019425751472990866406060745178201267888210392265449385908193,1\}\}$.

(76) $7^{283}-1=\{\{2,1\},\{3,1\},\{644316289,1\},\{182739497717749501,1\}$, $\{205902934361811678298102407850776182717715918337281553010436129$ 8126760824454698600268045597984639491824861889662493857701547885282574 3463384569393075657811525494018980546799598254701012863146448540067959 $3806467213,1\}\}$.

(77) $7^{293}-1=\{\{2,1\},\{3,1\},\{587,1\},\{15823,1\}$, $\{737305620346685452610890179165842772318404303441737795706818250$ 1781812795756403594118084649017691810978187751305157409426280909845017 0572726312667936273335791334222244969867032297622930275468789727720634 $6738438488327308295011451230700046301,1\}\}$.

(78) $7^{303}-1=\{\{2,1\},\{3,2\},\{19,1\},\{607,1\},\{809,1\},\{1213,1\},\{3637,1\},\{6263,1\}$, $\{250124533671949762689649229221474874676628240086834326710321934$ 8977261211314249290872113622297400983238054091221848413213952860927834 6483285416579124741617517032352627319438437181113141607115516500127860 $64898890375492487075375036575557409,1\}\}$

(79) $7^{309}-1=\{\{2,1\},\{3,2\},\{19,1\},\{619,1\},\{17923,1\},\{220400431,1\}$, $\{163288073459981092911015911012725583672896202807153549442940304$ 7190946323263119646461629801005446789341358508910933126672476042196489 1836653958609125022740991792042267923506388743680814495698839059209762 $59827795935697966768313068258679505800919,1\}\}$.

(80) $7^{311}-1=\{\{2,1\},\{3,1\},\{3733,1\},\{106363,1\},\{310379,1\}$, $\{904895224625893812988091213178336448427885830750199254579820207$ 5926830593856438218727198123712362081034991295942894065364300983317639 6455633249723589075290773968553785932225666553217320800343546877962394 $282558134170112622912472967513595460760710077,1\}\}$.

(81) $7^{321}-1=\{\{2,1\},\{3,2\},\{19,1\},\{643,1\}$, $\{859121000285791,1\},\{23884590227767303,1\},\{477280063808143381,1\}$, $\{877583282802140001932641556077928960670841896102746743335476661$ 6807440985568003608180342518051972841774133114526771709199423860941226 3876741731967024657616960716232656547073388025894443432191529410296366 $32000334190927,1\}\}$.

(82) $7^{323}-1=\{\{2,1\},\{3,1\},\{419,1\},\{647,1\},\{14009,1\},\{2767631689,1\},\{4534166740403,1\}$, $\{323880241032003815611424054193417184849407584561140218199717320$ 6276516917508471991862672908044771280670973237703703859638676446108725 7769643549274642275374272053418451975679564675745290760899763552107347 $30289807495655369230252665522194720183,1\}\}$.

(83) $7^{343}-1=\{\{2,1\},\{3,1\},\{29,1\},\{1373,1\},\{3529,1\},\{4733,1\},\{8233,1\},\{49393,1\}$, $\{734021,1\},\{1074473,1\},\{13473433,1\},\{83517610741606021,1\}$, $\{513140077902355851342749648661257956433045126418464938580294806$ 4367774703259901993060121856989531303372742276989977287777088529732507 8699422515610967309872822767053019367395599291739593443661018899591776 $121648725541902044825589426973,1\}\}$.

(84) $7^{345}-1=\{\{2,1\},\{3,2\},\{19,1\},\{31,1\},\{47,1\},\{139,1\},\{691,1\},\{1151,1\},\{1381,1\}$, $\{2801,1\},\{3083,1\},\{159871,1\},\{188831,1\},\{1446701,1\},\{402011881627,1\}$, $\{723461377501,1\},\{31479823396757,1\},\{430907693063791,1\}$, $\{376720035234076771,1\}$, $\{849138250161001102357523697384563489983132248356861435290718983$ 
9155674455325336922868252448825736138714840135092635324042980061278082 $6500783925034546954928397201454738596445364852203,1\}\}$

(85) $7^{355}-1=\{\{2,1\},\{3,1\},\{2131,1\},\{2801,1\},\{25561,1\},\{990643452963163,1\}$,

$\{112787554991591748609446118316967746548630991690591447185175668$

5490632594097525660306356133279367860420205822648680558621581778080725

6103372050556683948807495980871744846528142484058220756519112855262668

0636930149450198377036387747248338424169506260806622437554679861531172 $9,1\}\}$.

(86) $7^{357}-1=\{\{2,1\},\{3,2\},\{19,1\},\{29,1\},\{103,1\},\{1429,1\},\{4733,1\},\{12377,1\},\{14009,1\}$, $\{34273,1\},\{365773,1\},\{1175001241,1\},\{2316281689,1\},\{2767631689,1\}$,

$\{10879733611,1\},\{11898664849,1\},\{23992463461,1\},\{305213588009240737,1\}$, $\{467359137012519524745085559680145177955576162943849370422675752$

7545380691244773959907809874702898502514707910713081851858120570837398 $85436953356774953222032584307716650057149421897411512698692297,1\}\}$

(87) $7^{359}-1=\{\{2,1\},\{3,1\},\{719,1\},\{2051327,1\},\{3093863,1\},\{22452201769,1\}$, $\{399504166411930750130060066333350120458321100187652796764254466$ 1340392371765053820295350615233064691592099826565322042292209634978840 7558234059615473189861841278901449748850068530739850957509245062002161 8193131622344312301908097910841648846690567658842644750697896472086452 $9287,1\}\}$.

(88) $7^{363}-1=\{\{2,1\},\{3,2\},\{19,1\},\{727,1\},\{1123,1\},\{1453,1\},\{3631,1\},\{65099,1\}$, $\{293459,1\},\{1532917,1\},\{3191497,1\},\{12323587,1\},\{11795261559086963,1\}$, $\{294629918693044686536577670072041952949482099054592094102293885$ 0472637307592911440297452280017202573604595981604156780625944532985003 3012645476751789588307555286670643504684649488438359564418489045024676 $8668599410503263759386843085818528440739523,1\}\}$

(89) $7^{367}-1=\{\{2,1\},\{3,1\},\{5713457,1\},\{1232836358179,1\}$, $\{334984751006532638999044491536734803873372303151368396835965652$ 7753729565790949210627097330568856757176117295431570999797780110009347 2087167188336164696709263301535370447850236385953473729513224804853991 9773275058057737689763862070923808764007580831046256992824296786410399 $236230986324568019,1\}\}$.

(90) $7^{373}-1=\{\{2,1\},\{3,1\},\{2239,1\}$,

$\{123983411278666201465275824886878236062619639609393056374034439$ 0672549926340487645362799478663369621241108503516173197080189856051337 0823807426267871952628198206351615193956723736950307792274066753963392 73063069055522749918887003753235733822085824963766888706418342193399013 $948363838377348588249231750476876639759,1\}\}$

(91) $7^{393}-1=\{\{2,1\},\{3,2\},\{19,1\},\{787,1\}$, $\{493774760688250301025743342014503276925509144831235100252208497$ 8489036738064057339456047841654751845219451365647980146008919404707456 7583442241031066697971446985404262365898946325422380623984100640858305 1651654768336929258593693732157555883648978574995751233108862859799673 $145984813227829071059017052505318432532194083046261139,1\}\}$

(92) $7^{397}-1=\{\{2,1\},\{3,1\},\{2383,1\},\{11117,1\},\{232643,1\},\{7843916473,1\}$, $\{110010980972989993361557141323866023203663554796277769167636671$ 3157440212345503588231355559135637602354822704276850020342308140773926 1650874075262659232463446752142762476195039884132707150094613594505979 0253223646345808633437129955820952494401651878802257710137199785861040 $4003449859297709651129371099832646597169,1\}\}$.

(93) $7^{419}-1=\{\{2,1\},\{3,1\},\{839,1\}$, 
$\{247836664067439706199082599350319190850274222123522368953984623$ 0586223391782632962073387669776856234022072911851876546479087908633411 3244577967613559121918301723574964540298588081293911481966913577158462 5701521041375008572532138642782565388662464695867154911290362200968196 3529680256131393154036199315003078835077145645666828968565244527128015 $59864063,1\}\}$.

(94) $7^{429}-1=\{\{2,1\},\{3,2\},\{19,1\},\{859,1\},\{1123,1\},\{3631,1\},\{293459,1\},\{486643,1\}$, $\{1532917,1\},\{7524739,1\},\{12323587,1\}$, $\{44975113,1\},\{16148168401,1\},\{528808508322667,1\}$, $\{377341692572662931188133035082603892338658591856975927188200180$ 0857772357588561670469201162772876458402202499843337198950436034569654 2904615559023035416060154235134480496946296415286468604937100935461483 5050144792275811013076454937345992749437597454954587283969132028028201 $68169533928217,1\}\}$

(95) $7^{433}-1=\{\{2,1\},\{3,1\},\{1733,1\},\{885919,1\},\{341532829437623,1\}$, $\{268952162809985221897141157821615038103735770342502660185627855$ 0864245636463780705825308856812960359344093432790474339695446296470761 8157555946021586803829438876461023122960769310068225616286776285249950 9575935768721668064925236917874261311773153509683938727340398606803365 $801259364253633851530717295982026386127653883009134923078993631730181,1\}\}$.

(96) $7^{443}-1=\{\{2,1\},\{3,1\},\{887,1\},\{111637,1\},\{409333,1\}$, $\{982815371658598023237972434879107363433114542369440869126771930$ 7782722191043994331931766605162650888840368973259884112743817895835884 8069350540933211193625284873482169873829197802857616422679978234266830 6907462058631500227256517113617670241298941100529659299989657691574308 7524019479386122636167076271131930900315466637270313954034852598132950 $24090577680585991,1\}\}$.

(97) $7^{491}-1=\{\{2,1\},\{3,1\},\{983,1\},\{696239,1\}$, $\{213636046315531171819814770033633796110206120743960531746437437$ 6485985291118432663838669219902167547144328323526300584798640281891071 0546858670390310273388006263516494356000419713805588862656004125168660 1170886046472643280510837137774426320912787965970942528041786780248192 2511401732758931991537880767055653278144121627006794589416442754930582 $405031531750394670114684319306998076067984711534942456532108961,1\}\}$.

(98) $7^{499}-1=\{\{2,1\},\{3,1\},\{1997,1\},\{85829,1\},\{2841035543,1\},\{106026323287223,1\}$, $\{163255653904702308743046167822624592993565768865361681563884440$ 0394426106344357842428861331391395023982286977868793342236690338550615 8188516969394084182727849401951207666857193653675602683455030203593783 9912250286010618436362150695661923360330368584084850339923012224844656 0652174946481524138719734376770604120679141619192409314535600977012205 $68474769671043789091101816706361630173538326601,1\}\}$.

(99) $7^{517}-1=\{\{2,1\},\{3,1\},\{1123,1\},\{2069,1\}$, $\{293459,1\},\{13722816749522711,1\},\{33547155065898563,1\}$, $\{437269108109919626538333361896365344143689087264636569330106946$ 3405382659543262345694982837435574548747313096857923496074620684995811 1450301358032633775752806453688313336358078113980529591893333228095831 4268857093681019810116033683507937652851286079298017578110784036877031 6785249897133692679994509761401784813283249492207095276050479221724636 $7207085587662017641147704396296549902728834871729,1\}\}$

(100) $7^{519}-1=\{\{2,1\},\{3,2\},\{19,1\},\{1039,1\},\{9343,1\},\{8248987,1\}$, $\{147351359041383404073180336980537552978587366553021175053065971$ 
8214736337714733537726756138808016543579495190007747843403695929606896 0774189957714003631045024091527325056220428223624480795767219485645621 8037153493729623641021251315970706271146422877420191720937461294246832 0886124811313317253978827473301034162436595179222042823485912096805043 4873558752127441572879774160507917610231284250492734282844808663375205 $5002564499,1\}\}$.

(101) $7^{545}-1=\{\{2,1\},\{3,1\},\{1091,1\},\{2801,1\}$, $\{2156171040211,1\},\{2470529440391,1\},\{14551288401737861,1\}$, $\{266543445478275126210202011640794735935468745898133975974317008$ 0045842947967845111636142557649417651322461860294863738470446727797664 5057686996284696504879526519616358292975682016770610492897833301773976 2861759005943053334978808496228988999701696926256990952228470166830695 0096592799634754868524778401231534511894979434215650697325036360127208 $2232103124774546921074755968719748519837643020938568608041107549781651,1\}\}$.

(102) $7^{553}-1=\{\{2,1\},\{3,1\},\{29,1\},\{2213,1\},\{4733,1\},\{6637,1\},\{33181,1\},\{402592849,1\}$, $\{135151732264934206729504864995218031842940332378546653979092857$ 6164790629975110628544084036212010571734803988451399376303213732623957 3162012576439455892558353520725116375943892452877507335281300660208176 63161289806976871262586191167218008824123147470597436866896808626662490 4789248744045194447708183427026359987334469616395197685930585388000507 5159282346757950068349449729278843436220698244583132224007074525737593 $66946007551802054980126690237,1\}\}$.

(103) $7^{567}-1=\{\{2,1\},\{3,5\},\{19,1\},\{29,1\},\{37,1\},\{109,1\},\{757,1\},\{811,1\}$, $\{1063,1\},\{1621,1\},\{2269,1\},\{2377,1\},\{3727,1\},\{4733,1\}$, $\{2583253,1\},\{3368791,1\},\{11898664849,1\}$, $\{131595417902225378190845108794977413431720870526695171336243254$ 6290042674637870166928620009245766230661636552071056923168076336676421 4850958742935412625536692680366084168140243694207129500937524793734996 3429521824797862254871202982999628774729528044523381954113062699725115 8415501660754069621136920655197513337446866156883309819359370807673365 4508259187409337036700880448762779689142186004620457151014811086771236 $508200319,1\}\}$

(104) $7^{583}-1=\{\{2,1\},\{3,1\},\{1123,1\},\{2333,1\},\{8269,1\},\{82787,1\},\{166739,1\},\{293459,1\}$, $\{319591,1\},\{3896341085695040687,1\}$,

$\{750689448124948247816237094042250176504177915583992805848791674$ 1612233825150993960453409641227100348272808677440916262268104839050219 7295070456711529518084239208737425065939287987031414234336598675997348 5278269654930986770154567380871085723529409885868307011760087111669804 5072005528253710903997980150760916764842028545541009177237586686495105 0668290813588805001071976374912138261991688108477579556544115354626293 $50284597561249000264896682073,1\}\}$

(105) $7^{559}-1=\{\{2,1\},\{3,1\},\{2237,1\},\{5591,1\},\{16148168401,1\}$, $\{7823113057733767,1\},\{166003607842448777,1\},\{2192537062271178641,1\}$, $\{744612452058572976318327781749257232398433706414089279801654488$ 6309794285163450665215661369721602403823862622726148437248048472559626 2399942952877495218360398320252120994019479986310109402236300328877205 0651923238022669072258630626002794080633914281658542775790101476340667 34299682604167119559992721722868666191270484710423419316042184734892916 $264351409839043822642245804612432165252431028370927369367509,1\}\}$.

(106) $7^{611}-1=\{\{2,1\},\{3,1\},\{1223,1\},\{298169,1\},\{16148168401,1\},\{80614984399,1\}$, $\{3364901012227,1\},\{8715379679683,1\},\{13722816749522711,1\}$, 
$\{1975246678169248534933818223874358602312326155568254686556946727$ 3689052660641870862863272844304929321231644443543016068153998711475794 8829576955504907644728137087202737819970634475912642192684856999009365 4421042942354413972624303816216956397948798438098005122356386851312236 4400949541010677315073370928710414882448909059183274234335380996174705 0472169276820436157785323340431987063380541878501945934805757589908963 $88237035302721817488855194974439,1\}\}$.

(107) $7^{615}-1=\{\{2,1\},\{3,2\},\{19,1\},\{31,1\},\{83,1\},\{1231,1\},\{2801,1\},\{18451,1\}$, $\{159871,1\},\{267403,1\},\{20515909,1\},\{8736087301,1\}$, $\{126734235588747502855138501515705918224796131162528527045435276$ 7209258217453375321560378933987563415436844534721243844813090955132137 5506470103825555106316944986921061752623483369372539789008898639449521 6903480337396563107648299927210637220034081053653773728391794269455647 6507315553507454374509342125257550112953518924541833792261673818240712 3220242814199775141434509920655814905829798609439101804139222378553457 $266547940674207823930842955458419351336531724454430319748853581,1\}\}$

(108) $7^{629}-1=\{\{2,1\},\{3,1\},\{223,1\},\{1259,1\},\{2887,1\}$,

$\{14009,1\},\{308827679,1\},\{2767631689,1\},\{150757054187851,1\}$, $\{419980621112513304192306330823995821280066683350396866248395839$ 4605272988218758850071021172110108247488777454478722394336855948651077 4579242326929289195079790408061026833847139284389397891361888781405818 0271266456305964433949461582149314203272992713354799013368325453652385 3462609362089286065293930689330028114692169256401572549883367335054920 6631924707383271018772872845513924809132508021671581011032225760886874 7897314196451332685483164343619324822923122176992287446283093066597104 $991,1\}\}$.

(109) $7^{639}-1=\{\{2,1\},\{3,3\},\{19,1\},\{37,1\},\{1063,1\},\{1279,1\},\{7669,1\}$, $\{968299,1\},\{582053568181,1\},\{990643452963163,1\}$, $\{471266017668915946914465845185545449452039130814322125370206044$ 6907210013599683944055720253846530749120885278407880380867790232122168 2852286251981029815580066785442764250028215084778943407580603335742849 8277641309727702841005709560635904209557877385919705332743113249343278 63507397525282473705697408592365662105575833259689792566884301402806441 8471667418016592287300506292512856977292996210764652231315579947248850 1507977801649170495860300938806974662958373667651150138087133567071417 $4012833431,1\}\}$

(110) $7^{645}-1=\{\{2,1\},\{3,2\},\{19,1\},\{31,1\},\{1291,1\},\{2801,1\},\{10837,1\},\{31391,1\}$, $\{159871,1\},\{822161,1\},\{63350976270733,1\},\{166003607842448777,1\}$, $\{679668326738868015843129875427170473182525255856689586930814430$ 3221372530503323613205654575005180649641185541683714140659211707416634 5347560855071884027340400205462235843867106184462280338008545750762279 3399613847235546141291912567815183247729818924123007215374299324945085 1888127541937261305667689459997734998584007680545262959149090790356577 1083578160174790014872793179013467203007941395076226404762187818789247 4296098303447460991267296713529417735207605553929221153874712812465746 $9,1\}\}$

(112) $7^{653}-1=\{\{2,1\},\{3,1\},\{1307,1\},\{3919,1\},\{270475213,1\}$, $\{849748793036383123981364808737337709281070886985941254135396635$ 5493248438026964379224469439546371191838136120257678016407180190336738 0192567843849858771948655056966985238663305808740254044488352923807212 9324197195632675913072988542158875179519926906498052583376946076673916 
6224603711752135645342375310513590145838724730287813206612401408628306 2738962841489220932383245036689341715968467211032214134686688698041510 2462532986216436229923597279854042201376563486410117590931089109387150 $31808265096051855013316354250751345993382022559826369,1\}\}$.

(113) $7^{659}-1=\{\{2,1\},\{3,1\},\{1319,1\},\{5273,1\},\{282053,1\}$,

$\{706031095169933204062094887134964662176902494363276931963753011$

2554063996570435332285886722534242768040584051769314130234194130998954 3229088125474966919396518611007849111206630179359296123168940075069266 1024445119088876205798256486780016099586228848682352170731637675712768 9896882706187113637494011250062252908682830426575405521870330419734009 7188943236725647565634642103393170904730061106786544955502228305886287 2344734862658288180371190212538009606764201211883824439789278661568494 $7038541661557693475834084488295505356376634654474351221699787,1\}\}$.

(114) $7^{699}-1=\{\{2,1\},\{3,2\},\{19,1\},\{467,1\},\{1399,1\},\{391441,1\},\{79227874003,1\}$, $\{763527975915837345372727844865944590404083572316310215043076724$ 6974383210980226672747362091878350268126980480929278845045610612662980 3062700517815735712650910714217972060823187757737934157044122148610765 7755330924889617393361722473054112348270488298087058585154704193719925 6709581229578801293994385282688330331264423778015269770600851854855868 4387478786577135278626192393710920956567764783141202405523360562707585 4601111030007304334580477543475921638481228332626578229159214520958668 7822368613623406439997722181261183748861545482417381166541553218231533 $9755149665439,1\}\}$.

(115) $7^{713}-1=\{\{2,1\},\{3,1\},\{47,1\},\{311,1\},\{1427,1\},\{3083,1\},\{21143,1\},\{5451599,1\}$, $\{31479823396757,1\},\{3999088279399464409,1\}$, $\{640931687414161184785903385322136074480249136327973631586621238$ 1530323996784634794578225418083414826754732539487035076101194743462143 7063545890167430061132606588691492672398934129897274637803255705941207 9576453233904889632760944443012395144933735790816539329637572387049931 2435141803627972556708506932164483087712165587600949642858944761796572 2429279448674579103253129696987794992635708906167600181685958871330201 3723991772138517747893218220780504715987084382777915051255776712003094 $28821696782850213923220959938914282184569200870014459952617551013,1\}\}$.

(116) $7^{741}-1=\{\{2,1\},\{3,2\},\{19,2\},\{419,1\},\{1483,1\},\{6917,1\},\{7411,1\},\{14821,1\}$, $\{19609,1\},\{486643,1\},\{7524739,1\},\{44975113,1\}$, $\{879399649,1\},\{16148168401,1\},\{22931541257,1\},\{4534166740403,1\}$, $\{6957533874046531,1\},\{14975869588530371,1\}$, $\{108298732378534933789088359287735866001474951245023775391029716$ 0707687570398750140687694199769911248719060168580527791338491089487607 0535073592215052709699475110166812588202834303937461599218355936520439 5666018755396243674558787326350576568103566192362346411253090701319854 2856022747379537943904840802123689282409383953433437835266672646085357 9095278120794754792053950570759650907570403819377954231995456418917733 4549998869114400426008492178559246955680111563273494957356288353393715 $752118088392955261253563,1\}\}$

(117) $7^{743}-1=\{\{2,1\},\{3,1\},\{1487,1\},\{205069,1\}$, $\{11713923500359,1\},\{97986253240756675481,1\}$, $\{385135864714658907353934583309851755516134366646349055542017998$ 9695308564344402786454912806645885004619646676576819396515459064501250 8181872914035263846059114113080024278640510969085964321692867944673943 1877893486157222521845489434108946774532805680059739721785291790667501 
1534879827446614634240909436868204038086528412353759789691583826625683 7280611467094616570674307898873685128127623913110282718105745598654896 1705703611616717708558809915828472844769087463065469427444065882443680 5108086238931674921604477989398299201719821889132503842562365664933807 $165343914599824452988084725245261,1\}\}$.

(118) $7^{755}-1=\{\{2,1\},\{3,1\},\{1511,1\},\{2801,1\},\{36241,1\}$, $\{332201,1\},\{6788519081,1\},\{115356256117,1\},\{36076003832567,1\}$, $\{129615117850063249409028029604792628510020043125918208852361576$ 4293495450004503424396362121525124177697324077771954005149044954757141 8333427655954104221522409596898917492471953814326661638431808177703590 5914769393520872575626932612676899891766092459090545065342841113030884 1183978216703705889213423608382513303182036982630487096700913401303737 3314714856139900047928959963426678068078653523268850399425300800839677 1895577304108600939013635610398457377895216067638348617966008321444618 5957252581569544041784271835115685550247755811076187011349495530702384 $0698693348115675395124296540299973,1\}\}$.

(119) $7^{779}-1=\{\{2,1\},\{3,1\},\{83,1\},\{419,1\},\{1559,1\},\{140221,1\}$, $\{20515909,1\},\{4534166740403,1\},\{1253761670999103569,1\}$, $\{403150674199670165537040121423837975649488300269950963540862964$ 5483767682579967794585560627381062787670139482228143508032809375940200 2983866215180926914190411582440168312465175565155818661087707616829790 2141397606496350451847463182793332480563744896582374776181870696586656 3563113700680696403746151572640058101598974761071321469687240153138961 3595611858619117139243981798941948952610243756880945983443628231839623 2011261480355717401457577828975758907337740254206661481525663729517330 9988704835673824924304907977358746843779398800636997411663369841005850 $820478350857205702436692408220425313806450729752554813,1\}\}$.

(120) $7^{783}-1=\{\{2,1\},\{3,4\},\{19,1\},\{37,1\},\{59,1\},\{109,1\},\{523,1\},\{811,1\},\{1063,1\}$, $\{1567,1\},\{2377,1\},\{5743,1\},\{86131,1\},\{375841,1\},\{2583253,1\}$, $\{127540261,1\},\{768631951,1\},\{2576743207,1\},\{83226515419,1\}$, $\{71316922984999,1\},\{710074092185677,1\},\{196915704073465747,1\}$, $\{415831983474196104805174275626152034262137695646498991155957538$ 6464440146769846494389879409780484238969206034663175493596444106453340 74709508756666555119523677026857759057569826088015218028127177797525104 0667604341225814236664608309481663924470513181306502918491362814401171 4641987433742180225414239868721057112194668334558261983667701449055739 4267041135139566284973021829435249647973496626749600539739415034730913 4063614790267508818642994799186046625942527366276358633553678397949037 $569925949333392962378876972420598905199329347691581,1\}\}$

(121) $7^{785}-1=\{\{2,1\},\{3,1\},\{1571,1\},\{2801,1\},\{4397,1\},\{5653,1\}$, $\{1039945344901,1\},\{1263410268121,1\}$, $\{292637850687560867301661576750236411379685228882495094213408261$ 5881737031408016565703416127004122189676962543400691126395018467944328 7874840709854775004389708658604062139195431248778368822728044212941851 0504169616754773540652315525889498340630624145801855768818902369514611 6823717703327033868697925341612317578890264984828599217438815990629200 4099773145815323736348040348060403095451257614774066141406295831733293 3013201330887902307537917058080066727290016418603927128391549857552166 9648515969515052634944308437925297121346624875931155517970499843294553 0642920565689109967604545986589953183311343594240058544218367390466400 $71,1\}\}$ 
(122) $7^{813}-1=\{\{2,1\},\{3,2\},\{19,1\},\{1627,1\},\{430891,1\},\{6780421,1\},\{164514887,1\}$, $\{433973874722904244461669937388408309498270699336221221520466829$ 4552555100699358235671397833426625555856713268411447914165031115670866 0399285079467987205474821775538089480787172342643442455364958283367303 0177611083093661053116153677377082448059574387682966243001895426991304 1576476494676045025661445918452428916700455608548275160517777712666818 1751052531146897340878218736073482175601114766178712551935177108913799 5776167596186033089929705466273830871932413603976874200063259836863524 2697892517428121165656380705855275155058614584532715921503853719821223 3075528749973645014001537213391964087512648912953850135798375560382605 $26643404410454709282286342588367182587,1\}\}$.

(123) $7^{849}-1=\{\{2,1\},\{3,2\},\{19,1\},\{1699,1\}$, $\{25471,1\},\{644316289,1\},\{182739497717749501,1\}$, $\{176619623057721469494665905197712573170858228527234195931317596$ 1715248385180458725476382563595453077528090430415169066751989727332491 4437476333640928874112714139653712614137218165215142502557106396935684 8884180817376460752593443514212042763791134461183706105344577899910384 6244899110407521827145367037470014894496145986282341980510870674517449 1205784623592735742964035825661062354643716467400896679017514848674282 0679941050005893493477008829608598027056442900478169773807135348578501 0233101802347360681234893584538322862944701923987979297168063027335980 2218502595071383435563093065328403568313908048715070734528021335041976 $57843089232283369513777994982986881041423886873308458281953,1\}\}$

(124) $7^{891}-1=\{\{2,1\},\{3,5\},\{19,1\},\{37,1\},\{109,1\},\{199,1\},\{811,1\},\{1063,1\},\{1123,1\}$, $\{1621,1\},\{1783,1\},\{2377,1\},\{3631,1\},\{3727,1\},\{5347,1\},\{173647,1\}$, $\{293459,1\},\{1532917,1\},\{2583253,1\},\{2904661,1\},\{3368791,1\},\{12323587,1\}$, $\{27170749,1\},\{57156633859051,1\}$,

$\{712383793896664420694508055707541026895532017325610737876304833$ 5257028028592336278603208240152505532546859443034108019381968285987534 7625737942824984475921928934624941799812104269496229396801974189139950 4638914671075058594961033339307926614929686768297886248628777627646595 4021973293919904183799308758827670033164751267287366567611896881770308 6431336906265018730947849113560863635496830558582063864199847849415461 7278886015697605419369522578801409409716945403152614811261161039962106 5320222524717429137386500274956588616391113957339529533482766158816466 1970630920478069425960126590366373039480689864563423359922778802860859 $75820104877141459098825681,1\}\}$

(125) $7^{911}-1=\{\{2,1\},\{3,1\},\{1823,1\},\{32797,1\},\{382621,1\},\{10664977216349,1\}$, $\{523375850555417066359627992485313473766730966562254880741120704$ 3714546473895334713926465701979213242468843184918648407395875112521053 9189287576339148485175593578998505570448552591557742513594608294113407 1718738039876862956809193547896574170988493195571435544460922125742959 8839224040540648811404874329189416716305642854438943370504879800904778 6829111984363480925105101647937864763639685398479942677027232306381872 1340503853779404678262192250718044514905243953162016352647188620096769 1996141346759017899565120585923949098120646227376979816068207320647839 9451560009448367965089144626205422289161663125941562405239340798335024 0352002066751752817843397332329790726622749157568945127229692768490947 $31367504684218070896687044486076291983388379225643,1\}\}$.

(126) $7^{923}-1=\{\{2,1\},\{3,1\},\{1847,1\},\{34055009,1\}$, $\{16148168401,1\},\{11112633233131,1\},\{990643452963163,1\}$, 
$\{158064435094761907685095513114240511793170518770509622837632485$ 2922748853915824501117732843729666710232182184729017900477295994697997 7250579963977283282947195487484574872261813904876432221394508307611995 3724658898063134437674563274052798516964424308259012896506301775793788 5972695626606235271195436057124302906623285856816912606479788435577215 9102830869189580056675332527651165124527170590916062037267244721330475 4621452945171118003388441635593728811442604177010851526909159787306739 1958128763741114314342276332523158504280243351998029835755104479681890 3246910705171219759747916121308593184358681210535126798642133335658368 2034463335426878938609969754904221325188343847277833313861690169098413 $69682810129960329495336812933851318503,1\}\}$.

(127) $7^{933}-1=\{\{2,1\},\{3,2\},\{19,1\},\{1867,1\},\{3733,1\},\{106363,1\},\{310379,1\}$, $\{380679956994670592853283063915754932979660111721703293455451513$ 7022960743464420609390180528124824805348932740897145412379343168964486 2217366235735834723718350238493382746589263325753348584600617300599541 7690171815737585651073075788807262714318410007214883190205502380380973 5763216507188479361132656449053837244037073260485241031036386576336027 7454777037098451635164870200315306329735355183906320658537590218617442 1905531162039956913047758100760007399951572163326273249322978345773492 1060175498135349860313449742472388119227504896370695064659103778541628 8979602955723143679845011276640038207172475133868935375348524419070167 2791843490717314240677265606586954524828193989161569606339439460906177 3485807373608696141218434629475564025591616911104439001626702463406347 $670919,1\}\}$

(128) $7^{939}-1=\{\{2,1\},\{3,2\},\{19,1\},\{1879,1\},\{50707,1\},\{9450044264774621,1\}$, $\{114447135581366713082578980606578212845180629562906779241097648$ 0925652120970967270778866452540981280385535104031053815252829305575834 7753142255131437647132927549853250467508632342704354013924725688314465 8408791411428928546689306660518897520988201731644651313309465734844788 2549140322341006941797033959638941813392991025502841637634037292644419 6690891509188257394712665398178999512872886015734551605437590300093731 2614462404465839839855393039154672722757321224589279598837681361635889 8941865476621532309703875206137097144298048277076538428780919295430436 6639621113034064657491026050393700481954803112145487681517693834986936 8339788716875352764741580652464963154392945916864171634937237257114802 7854584931474533251694821663517245263489719807387787416477992219298381 $58177,1\}\}$.

(129) $7^{953}-1=\{\{2,1\},\{3,1\},\{1907,1\},\{32778579857,1\}$, $\{637294332766552047134795973179850474042172459956121404172695173$ 5621796256876446534877988501967599464960803080336314194252004438354628 0179934824302540125114315713824652869380915812251630130263920381736447 4794414406535212952833043231130078924950533576320029500584007545571074 9646575026413290577281490171095872179968215142130391244887871797247811 2327529776804818830836222308279495295719763981625077510315577331814200 6792838484166460859631936918290935528762210153810544315303370144867292 4325478484310130081466150351550867858366626515985904714792273066760991 4091940043148336356881894244122186341986187248367654606981985184395337 5857017257996682883172019602937375392157898975968901284514327302854420 3683416126758024577531965293084276353460621307402667428210589382878518 $4922432525705688364676376299,1\}\}$.

(130) $7^{965}-1=\{\{2,1\},\{3,1\},\{1931,1\},\{2801,1\},\{14669,1\},\{627250310113980671,1\}$, 
$\{110795108594697691391285390088639305292922074143911879562146792$ 5383769734472237208710298791106105163916558010616406444591747683447678 4714448678638465429323733543809109059998863507655409937320330164026685 1699258079342007106355972260884634814301357494666702421742867788155739 6109042955338572579446183125224475321806005162606104858041808470146275 2028088765399423173583615156563850339520411589193178120718947572863347 1938219805503077394982856718906254852839998500125941618688401940850308 5535838684271576237310450300445566193214036576432576043730249277783555 2003428619312542623581416371283821300382424336461445745133760202489348 17301602576482969145394414659265063446086157569091458866465618498386076 4077861933825647004296543797880166207047993359049805325803157178896554 $238225886297432449426129,1\}\}$.

(131) $7^{975}-1=\{\{2,1\},\{3,2\},\{19,1\},\{31,1\},\{131,1\},\{1951,1\},\{2551,1\},\{2801,1\},\{29251,1\}$, $\{157951,1\},\{159871,1\},\{486643,1\},\{787021,1\},\{7524739,1\},\{44975113,1\}$, $\{567538141,1\},\{14046059431,1\},\{16148168401,1\},\{3960157950451,1\}$, $\{4446437759531,1\},\{31280679788951,1\},\{434502978835771,1\}$, $\{163639934079213693740168151783708511290067217129148380475137912$ 0685466723386311195174756660665224493987484429364852712710842569232293 2461399282643956712594938508286074037178498798090246281631336576743101 3100792158227912398654231361581623042219479124050856272124180181600630 2339314657658835961984525386418409440484824099466267299771550838146450 7571627184398051520453364871408494715407683097917032883783149679005427 1277516088655604592812895581772998304872513693678152635849085307729897 0181976349553347114604615360432775548134616108509443778405463612450518 4108507970527263750425972435081081333381761897862851060956923505778817 $24756693500272757965794897324774920189097214487661396459750981,1\}\}$

(132) $7^{989}-1=\{\{2,1\},\{3,1\},\{47,1\},\{1979,1\},\{3083,1\}$, $\{670543,1\},\{1501303,1\},\{31479823396757,1\}$, $\{116242749546290070635225720313398222365731449862421729706637003$ 0131838834638292717045289561535399292325969134548125225718548199759304 4241831730134784002845360917435077570925321548504360697283392436976244 6750030450891705435139725277745020924139436316846440638310591301085733 1355981295687725874989518494853793250166407270489060725643832954491729 5457877660726692960376765653509626783668359015106730747941714618178859 0482445199715171541138637445887437655502674374751758470708005727910292 7841780997254146601616265984028751247728954213565936009761029051975978 3488446632223408497684022917882288033384978048553418678454039934722874 1873395514135199879692270400403260611221687220935429656527881704197565 3533942074788560238263917773583090593899701123824820142696422616251228 $731411685529924733153294617803486379523,1\}\}$

(133) $7^{993}-1=\{\{2,1\},\{3,2\},\{19,1\},\{1987,1\},\{7283,1\},\{25819,1\},\{66170604553,1\}$, $\{179976139119387795239433391101349117722352295579878515570857947$ 3461927122114750152573472049905516505428948127573947256445238234614165 2285041995301335877757032895249226692319165859739545164377599232935918 3834858032256556573863106796426279204744683489208814168763876419188153 4701467787470798878651420787587312084389136822216201318361765169519584 6557221575803546851775510607413776814199403224993314361993705246343103 1713314777896257070615893594884264796128212833199576345796006854490163 0951785705208210824305231074322508025527332411183042854350551567316977 7006559991603096763565541340661862377867046820408146845689846244756605 4342058365116898836137799288763398354678985394384773786941931492042710 
0233939204532341399831943813100937208964873796405963120216831765697273 $2093908364880117290309116960996936146743793819871819,1\}$.

(134) $7^{1031}-1=\{\{2,1\},\{3,1\},\{2063,1\},\{285960223,1\},\{871749679,1\}$,

$\{640813058375167219449763593588812763167792559500329558015768558$

3154271065797312389797589383732158068642085443426448207905982655165733

7073129304834387194211659300115388372369477620481832796836077250352517

2108246509473012886090589002483932420055555865904049634334260247602541 6174461060508950825827403615817243499778432386753785896338544257957551 4075476522229495170553655253169462767447693354028717010689726202058332 2570465311380286472464244210079472945111312905223591121678045313360525 3027420106486526448956039479541449773502837359756882377517023014215110 7474274303808769699971018929725648361246956853505106230797542104928312 2137859453491989078895603147962418386605352961551527710184377891501724 0351512945439081080368470508356231663477826217228285817192473197132731 5493381699554002612073538060405393059457028337929522737026390081928998 $33179449277333367,1\}\}$.

(135) $7^{1049}-1=\{\{2,1\},\{3,1\},\{2099,1\},\{48992497,1\},\{420164363,1\},\{284330642001457,1\}$, $\{436825347906424797401289373041438960887095681951458264406595082$ 9378119871598869227232939600341115409793699165095703858112429161713674 7023659125040143436617116791718564639915983118949234877707325789447055 5246508840774196675925899257568853893256815256458002192821250373363909 3703619766166127806528021267867637395000292463244002730666595356973122 0587972839825824530539760447040343821563055063925553752147809989939264 5347035545097834388399669103675511711017720567977042518548188006995217 1290319100913444165962731267687744107152596203803113937289136338767816 4188286244417091635649138083757100509015688942271685324206391375783404 2300625512774141178135853953501353936140843150667266554282812827211578 1312765037930747726143242489229379641044179300963334162062135023268108 0493992916600271920044808439645793493842916857998671573120662868858946 $1062105651324877137,1\}\}$.

(136) $7^{1101}-1=\{\{2,1\},\{3,2\},\{19,1\},\{2203,1\},\{5713457,1\},\{9127291,1\},\{12088981,1\}$, $\{1232836358179,1\},\{555169875729277,1\}$,

$\{8728499082904554302545956605251578587262586261753436555889104626$ 0621556438728200613258087030148119378717267690876278420982644910307584 0351971975008253249999709879284802086691673810649367769374685715694678 1642316153894950827730914493830619262722442627531778628717593401700067 3064777276022180886658401609599261717349609469660171824624734105979353 3627224068700327850837546357816522191932789188321809908250711517912967 4226510880139618299070832429290204913736789170254805950325833598264670 7438536341511265720370259535864556576327671429633588661925841883872329 8357277321639699949176934350713270146196027044843816060232896622387054 7403763629120470527947791286252644382250304077180292619873793572126352 6138829533781270640488782058899534112933771233119688270061566527071124 5207437580882115816264199971817007276825600598624548978731748472051849 $80173442823952053900286823502259807616826331,1\}\}$

(137) $7^{1121}-1=\{\{2,1\},\{3,1\},\{419,1\},\{2243,1\},\{8969,1\},\{459257,1\},\{134927809,1\}$, $\{550413361,1\},\{4534166740403,1\}$, $\{289481342287376532691829948925003693983795819012520488989672831$ 9094718147097855569042441423255350241361917004176284294355641771205918 1081986428070569022658888002207647015720366769127542307384823609386550 2171451726689993548951096093181401240734493246374491479647065804472268 
6138143023424459405800054825051589529511151410077910950642982375635939 0964301730595782322608142789271752369308028040005012173774506667723197 5358545618935512731588760773775894851625922012983093784637813732140814 0432298626232587470076609572980856157191221221375322213291426016572738 1338202349439363653805326801233164749453838955763788998341779388630823 7782621588090155765400170352298300161903730950788634490992245624996707 0892106702062444641929419144432200609875327952773987561903484787887552 2373063813188809931735562563044584017632155947832239296378162380009921 $502773559492958544285808213753631441426691181541231684644283072153203,1\}\}$ (138) $7^{1133}-1=\{\{2,1\},\{3,1\},\{1123,1\},\{2267,1\},\{17923,1\},\{293459,1\},\{344210899583,1\}$, $\{113321953624108734148501587859108700112951593165094394602122174$ 2085179619773833454298336344980860257105288813529240951536823161713287 8603429559620203644586476956594729892598778203353680394916813882952869 1392064539738496260451861255316451892592584621949970034526705054502109 6914598373692681791224841579538953152705210257382188908583239236619651 5940429733504513181472830574120712855213354392383721463255662888425788 0546392570314161902741929614330238714153852095447231081315914923597068 6471566616073694933834473141025344224212561740683120626837466106584749 8394058798291354224039633997726551506265957774744267087318257070923024 7783287308549135790956428285574541169829616723688169479112314889908075 1135665473645386366135736152889016377681603501281426739356730475725154 3391644279656425503401715249076968927606432967976089325247048939206942 9503306769363915243765841053475047442940889091702624019695704077176638 $443773214296469686816814231,1\}\}$

(139) $7^{1143}-1=\{\{2,1\},\{3,3\},\{19,1\},\{37,1\},\{1063,1\},\{2287,1\},\{130303,1\},\{2208277,1\}$, $\{10920223,1\},\{260461507,1\},\{9809667961,1\}$,

$\{11699360074183,1\},\{3451948895547127,1\}$, $\{295833553111890954448612314093327906546577022386017975692244985$ 0928004739744048055261444047972549962631885500379716973033115286228724 8853056544418521802908869281010902238238746889432995180028912089446372 7528805380136624347334558793667584981389441900209443237645424545985354 3046186759643498083262960988764147527952796892706626337543646849079561 6425193696882898176500309357583940049466890280046025517211835100637816 6974752695164801106903971405633971030527754036587643595825193115170207 9590606982008883097581680623216753373745545637888375058449399006746009 5946759330948212706616071376698102857979522884139730908777892378894674 1087926233918077656443998959854088836815761508505528993621189605191555 0133806379359566599324682486105743827849058795499621811934050813819402 6159353044169298136013271921128218790519497759392736541913125580567596 $539496022305173951855891770082242411345106699001420373921,1\}\}$

(140) $7^{1175}-1=\{\{2,1\},\{3,1\},\{2351,1\},\{2551,1\},\{2801,1\},\{77551,1\},\{138711101,1\}$, $\{31280679788951,1\},\{13722816749522711,1\}$, $\{210063937677829240482609778912761612020923670468129558980782110$ 8676376216803652173847908777443876027871384631031676802192588116602210 4079976536754175236154473480555658182514436736190188005263064151102060 0769004001033031496387090821018302543746168747450529753244466885609384 5530946632372598747262413070184584093090731720202967225286876744320577 2967407039846239554139279153326809251991857317111887918650177869410049 0284378516888218171891248660750631175055595558508893803488199515986488 1081195251082914939593283201651824154980967582824922119647365896170406 0366235306818554185446002019922355159040825893793823053779883819169374 
0593430405467036578596179490544181727990953251878701742516841549480063 7405233193364442766013168296626956124967956828362718761368220751570410 6212234471601312103237350998470909097998739547432269179443283344250951 6853624193713513904884117463547027070633231799407418357752365317072489 $2412271700280514331716414396502279787,1\}\}$

(141) $7^{1199}-1=\{\{2,1\},\{3,1\},\{1123,1\},\{2399,1\},\{59951,1\},\{293459,1\}$, $\{1583492923,1\},\{2457808519,1\},\{38793043519253,1\},\{14551288401737861,1\}$, $\{299795689062637983704249728058685524733298287883656536648078247$ 4770136752367329062687529364482737701175421615127173474927122160279360 62165096625151015621945311099084871205791176488774133885550085527837495 8512515889819833081166032050227404189975793583830429241661626851141009 4641842313641522735702683520336260527742078237314147511005284290784955 9302860526514428885202610014429565938939802010715698345116208376011855 8088674811324990314818356380230217419218144789164032905127193667043092 1575239278025273514371969213854599086945819456921179822733719616922466 8226212135764764466676103646237755489492035468765298436833748461783309 0471216990680614719483896502945835825566395109446192378873371921689272 1573262521320774628510437217950876113128178310780880647642329345575208 0731219737786555970212419779433770638372509398257202649393244109295030 1684792442943187139140241244625090960123510546038397123746742744650014 $402943673578806851167892604968329424970860269,1\}\}$.

(142) $7^{1205}-1=\{\{2,1\},\{3,1\},\{1447,1\},\{2411,1\},\{2801,1\},\{28439,1\},\{595271,1\}$, $\{1471180803061,1\},\{33486078253526557,1\}$, $\{98544033012195431,1\},\{580351872045743557,1\}$, $\{788001439410563834670596313758909272514128954482820092062783513$ 0192529880516274450648722239947115081550028544445092274701705996936026 3952139550327990097559765599730102955830977428267731112966492919245803 0390455270975558863529020294474015266670930078177261061012446415774435 8196200052959993507638938322214790716198036929811964619099943989370719 7663458607378690513024503857733119721476063903744080959634768360131724 5265344292318804867768980954788583361338952031491535094834948909521391 5962762668999609455607060522009233758797546923220352583625024538449801 8121414178850613034259241271715843955820230100565073593239447362201863 8595429501038128407435350791200359216490950343746056026446659476319150 1427438941908854200686618956523875734031219269601793715470783880408067 1782771543233625114242749197784689485030870974396521363532704984034889 7942939842687831822880222901904937094739086686131645691670871050793801 $7331189363226777538374225661943,1\}\}$

(143) $7^{1233}-1=\{\{2,1\},\{3,3\},\{19,1\},\{37,1\},\{1063,1\},\{2467,1\},\{2741,1\},\{213721,1\}$, $\{251533,1\},\{443881,1\},\{575401,1\},\{9712753,1\},\{44807221,1\},\{109781389,1\}$, $\{329437051,1\},\{27500724319,1\},\{8350989090751,1\}$, $\{748462250678523518049517456077821793235105640889442254843797349$ 2130808709118007170851869798035153873029468042251699417376580973596096 7138759506033392185534668445576218515502008906669094142836764844622442 8182192504002034282542832223931987197650259392783449319738712666900643 0971750186303349048943837423939277083401757122840463876099145798628936 1805637095131104971998640438917987369335084761801082309047102582376364 7382216363233087789210249592779978762626280296917990065384000347544703 0722796882541703851744122470606029371127569844588927367141961826283674 2673473512799524007946308558839130075516332180087328157328692522158583 2717341792716761318719533723120991928549921200336234675920916761615354 
4632928749024689398931212271521547766134325611234411977913499694495044 5525788437443773548219999649461554558590181395160568353215685660027415 1601974498798869987073338087892180324801599531249597434514933046649381 $224638307375303346152075195264096947223082424697,1\}\}$ 


\title{
Appendix G Factors of $7^{t}+1$
}

\author{
(1) $7^{4}+1=\{\{2,1\},\{1201,1\}\}$. \\ (2) $7^{6}+1=\{\{2,1\},\{5,2\},\{13,1\},\{181,1\}\}$. \\ (3) $7^{8}+1=\{\{2,1\},\{17,1\},\{169553,1\}\}$. \\ (4) $7^{10}+1=\{\{2,1\},\{5,3\},\{281,1\},\{4021,1\}\}$. \\ (5) $7^{12}+1=\{\{2,1\},\{73,1\},\{193,1\},\{409,1\},\{1201,1\}\}$. \\ (6) $\left.7^{14}+1=\{2,1\},\{5,2\},\{13564461457,1\}\right\}$. \\ (7) $7^{16}+1=\{\{2,1\},\{353,1\},\{47072139617,1\}\}$. \\ (8) $7^{20}+1=\{\{2,1\},\{41,1\},\{1201,1\},\{810221830361,1\}\}$. \\ (9) $7^{22}+1=\{\{2,1\},\{5,2\},\{661,1\},\{1409,1\},\{83960385389,1\}\}$. \\ (10) $7^{26}+1=\{\{2,1\},\{5,2\},\{157,1\},\{1195857367853217109,1\}\}$. \\ (11) $7^{28}+1=\{\{2,1\},\{337,1\},\{1201,1\},\{2129,1\},\{517553,1\},\{515717329,1\}\}$. \\ (12) $7^{30}+1=\{\{2,1\},\{5,3\},\{13,1\},\{61,1\},\{181,1\},\{281,1\},\{4021,1\},\{555915824341,1\}\}$. \\ (13) $7^{34}+1=\{\{2,1\},\{5,2\},\{137,1\},\{59361349,1\},\{133088039373662309,1\}\}$. \\ (14) $7^{40}+1=\{\{2,1\},\{5,2\},\{661,1\},\{1409,1\},\{83960385389,1\}\}$. \\ (15) $7^{44}+1=\{\{2,1\},\{89,1\},\{1201,1\},\{8713,1\},\{8206973609150536446402438593,1\}\}$. \\ (16) $7^{148}+1=\{\{2,1\},\{97,1\},\{353,1\},\{104837857,1\}$, \\ $\{47072139617,1\},\{108604397663266369,1\}\}$. \\ (17) $7^{50}+1=\{\{2,1\},\{5,4\},\{101,1\},\{281,1\},\{4021,1\},\{13001,1\},\{25301,1\}$, \\ $\{38327966300231909291101,1\}\}$. \\ (18) $7^{52}+1=\{\{2,1\},\{313,1\},\{1201,1\},\{85094881,1\},\{1377454635342537460935008154217,1\}\}$. \\ (19) $7^{56}+1=\{\{2,1\},\{17,1\},\{449,1\},\{673,1\},\{169553,1\}$, \\ $\{39648001,1\},\{21535258550401,1\},\{142256806230113,1\}\}$. \\ (20) $7^{58}+1=\{\{2,1\},\{5,2\},\{233,1\},\{136853089,1\}$, \\ $\{55716067510309,1\},\{116714640028973541741413,1\}\}$. \\ (21) $7^{78}+1=\{\{2,1\},\{5,2\},\{13,2\},\{157,1\},\{181,1\}$, \\ $\{3445182122061576024844195480886675473399933083881531303593,1\}\}$. \\ (22) $7^{86}+1=\{\{2,1\},\{5,2\},\{173,1\},\{1033,1\}$, \\ $\{533721886283112766162388808829964093134556068537042973846397895277,1\}\}$. \\ (23) $7^{100}+1=\{\{2,1\},\{41,1\},\{401,1\},\{1201,1\},\{810221830361,1\}$, \\ $\{101087819444250339960209489699235199115942108498312937463657783601,1\}\}$. \\ (24) $7^{114}+1=\{\{2,1\},\{5,2\},\{13,1\},\{181,1\},\{229,1\}$, \\ $\{814233839701286841407600426610905218940681274414001855960390665$ \\ $81389479689875320467177661,1\}\}$ \\ (25) $7^{120}+1=\{\{2,1\},\{17,1\},\{241,1\},\{881,1\},\{169553,1\},\{542081,1\}$, \\ $\{33232924804801,1\},\{2312581841562813841,1\}$, \\ $\{5061247714620122417251446860692932425823228722764561,1\}\}$. \\ (26) $7^{126}+1=\{\{2,1\},\{5,2\},\{13,1\},\{181,1\},\{1009,1\},\{250993,1\},\{2304793,1\}$, \\ $\{13564461457,1\},\{13841169553,1\},\{31864919689,1\}$, \\ $\{195489390796456327201,1\},\{378063753860405247739880147553087649,1\}\}$. \\ (27) $7^{128}+1=\{\{2,1\},\{257,1\},\{769,1\},\{197231873,1\}$, \\ $\{190845781233779568947114378127612565912345052718201774886297099$ \\ $41792454027327157612573467077889,1\}\}$. \\ (28) $7^{132}+1=\{\{2,1\},\{73,1\},\{89,1\},\{193,1\},\{409,1\},\{1201,1\},\{1321,1\},\{8713,1\}$, \\ $\{532489,1\},\{98138029441,1\}$, \\ $\{482121267192714407437280028916490787579914240064587528159545948$ \\ $6484414160017,1\}\}$. \\ (29) $7^{134}+1=\{\{2,1\},\{5,2\},\{269,1\},\{3217,1\},\{4591913,1\}$, \\ $\{39144122221793,1\},\{27982218262152281,1\}$,
}


$\{804306663862573118378356240292749022674214720777202165810697852$ $844101,1\}\}$.

(30) $7^{138}+1=\{\{2,1\},\{5,2\},\{13,1\},\{181,1\},\{829,1\},\{21529,1\},\{26497,1\},\{26681,1\}$, $\{649981,1\},\{15513961,1\},\{5755716973,1\}$,

$\{487795294255062097167753810284203040831025519730964911030937505$ $5914548053,1\}\}$.

(31) $7^{142}+1=\{\{2,1\},\{5,2\},\{569,1\},\{853,1\},\{29537,1\},\{810253,1\}$, $\{173741413084695514697186489009077890080494674659824796210993409$ $5631917002616726770787354278635009866473,1\}\}$.

(32) $7^{146}+1=\{\{2,1\},\{5,2\},\{293,1\},\{20149,1\},\{36793,1\}$, $\{223078399569228140808235717345501553739210275293675092238683688$ $084402056924870062767172694258955411407146512673,1\}\}$.

(33) $7^{154}+1=\{\{2,1\},\{5,2\},\{617,1\},\{661,1\},\{1409,1\}$, $\{5302529,1\},\{13564461457,1\},\{83960385389,1\}$, $\{804952974589975605271303169284712837112899485096159546067244817$ $83541711761172675099569755257,1\}\}$.

(34) $7^{160}+1=\{\{2,1\},\{641,1\},\{7699649,1\}$,

$\{134818753,1\},\{163344191041,1\},\{531968664833,1\}$,

$\{142097814329729959157191760967108795941606877838729187172485674$ $75215750584159203240471457715521,1\}\}$.

(35) $7^{170}+1=\{\{2,1\},\{5,3\},\{137,1\},\{281,1\},\{1021,1\},\{4021,1\}$, $\{59361349,1\},\{15425560249042501,1\}$, $\{128290761593369346640656752099676373244020397780199865772887889$ $90444811691865608161310987056313352306899829,1\}\}$.

(36) $7^{174}+1=\{\{2,1\},\{5,2\},\{13,1\},\{181,1\},\{233,1\},\{349,1\}$,

$\{136853089,1\},\{55716067510309,1\}$,

$\{152774412550304154499976319449661669094591663391286688440608267$ $93893488209951205979084398683456973501189197527509737,1\}\}$.

(37) $7^{192}+1=\{\{2,1\},\{1153,1\},\{35969,1\},\{55681,1\}$,

$\{12644353,1\},\{1110623386241,1\},\{2474441815297,1\}$,

$\{113080655548928099034032445260779110394683137640279688986449751$ $33006042944929223059791867913583416051929003284243949313,1\}\}$.

(38) $7^{198}+1=\{\{2,1\},\{5,2\},\{13,1\},\{181,1\},\{397,1\},\{661,1\}$,

$\{1409,1\},\{29569,1\},\{257401,1\},\{2784937,1\}$,

$\{678240817,1\},\{13841169553,1\},\{83960385389,1\},\{116320403133001,1\}$, $\{252561853778422700978578305180968432370632969651396656080904012$ $6733269201040907313372303091533,1\}\}$.

(39) $7^{216}+1=\{\{2,1\},\{17,1\},\{433,1\},\{2161,1\},\{19009,1\}$, $\{129169,1\},\{169553,1\},\{455761,1\},\{25726609,1\}$, $\{51385969,1\},\{635311009,1\},\{338325042961,1\},\{33232924804801,1\}$, $\{609928137331215931325743981240140163846633907421573770915524903$ $0236383102520548972645581362856247870137873,1\}\}$.

(40) $7^{230}+1=\{\{2,1\},\{5,3\},\{281,1\},\{461,1\},\{4021,1\},\{26681,1\},\{649981,1\},\{15513961,1\}$, $\{48203401,1\},\{1853224081,1\},\{9118947413041,1\},\{55681942361860463813,1\}$, $\{148380801538465137230106248713887408397705874784316169458289482$ $226910803482493259028339119907427804070043125754977821,1\}\}$.

(41) $7^{244}+1=\{\{2,1\},\{977,1\},\{1201,1\},\{2441,1\}$, $\{279181359188709713738914433888025302486858307167679087718299549$ 0630951285104641635563749811425948601767651894624464762116116461867852 $0760666493897822366899888278279230753986537753226380807806637993,1\}\}$. $(42) 7^{254}+1=\{\{2,1\},\{5,2\},\{509,1\},\{3049,1\},\{15241,1\},\{23128733,1\}$, 
$\{165154969792908878964905702171414995584595891837393457853668730$ 87744704695018672013258340367450667515014783912648886664691652498571181 $457376599083272732606377874814039093046414151557343329209409849,1\}\}$.

$(43) 7^{260}+1=\{\{2,1\},\{41,1\},\{313,1\},\{521,1\},\{1201,1\},\{85094881,1\},\{810221830361,1\}$, $\{480003171956223529669924055211056738057773418463805298730047823$ 5836109704015636304685106145889317907823261087555922161075168527065033 $810660211151300206528306837101541526983017467890835617377,1\}\}$.

(44) $7^{270}+1=\{\{2,1\},\{5,3\},\{13,1\},\{61,1\},\{181,1\},\{281,1\},\{4021,1\},\{9901,1\},\{26893,1\}$, $\{68347801,1\},\{13841169553,1\},\{154625887441,1\}$,

$\{167039577217,1\},\{555915824341,1\},\{2295769290481,1\},\{590297313273013,1\}$, $\{755463210554036658915233148466172822754547618293087404662727832$ $2370361975704921396754516475617944257276206903845753955042539181,1\}\}$.

(45) $7^{282}+1=\{\{2,1\},\{5,2\},\{13,1\},\{181,1\},\{1129,1\}$,

$\{86857,1\},\{95881,1\},\{4574857237,1\},\{23146232881,1\}$,

$\{177405126822295090374135014406451784279375509548214737594895898$ 5034697350054321705425609884506325995277278744003670501725597568651468 $04863789194255265263574825331541511769200218595589069995280171557157,1\}\}$.

(46) $7^{288}+1=\{\{2,1\},\{577,1\},\{2113,1\},\{7699649,1\},\{9917569,1\},\{134818753,1\}$,

$\{596878081,1\},\{16356157057,1\},\{531968664833,1\}$,

$\{187521384109685338945221918793894283983642255950664916404230950$

2518236429244063009068272341167688352584801206501887858699863790733380

$5480760496653511449018481339543611301976909064254337,1\}\}$.

(47) $7^{296}+1=\{\{2,1\},\{17,1\},\{593,1\},\{1777,1\},\{169553,1\},\{15731809,1\},\{274166594399233,1\}$, $\{537898242355005363187080995372272918566624712854651450653774432$ 2860468093101928150451804702426401669342197467279838010286865747038545 0233182538192600698131831590942560194904424902429808259023614766264343 $4918210070753,1\}\}$.

(48) $7^{300}+1=\{\{2,1\},\{41,1\},\{73,1\},\{193,1\},\{401,1\},\{409,1\},\{601,1\},\{1201,1\}$, $\{100801,1\},\{12913561,1\},\{810221830361,1\},\{85560261859655897641,1\}$, $\{274186405564458718822730366656762022450762143859152488613621009$ 8214372367903385699545490402166104632279949424133493215602256993670017 $804664203292362084118703404079394610084356510519536806170201,1\}\}$.

(49) $7^{324}+1=\{\{2,1\},\{73,1\},\{193,1\},\{409,1\},\{1201,1\},\{1297,1\},\{40177,1\},\{42409,1\}$, $\{137089,1\},\{7821361,1\},\{32952799801,1\},\{317328896377,1\}$, $\{189027978606574721147194185783957778308052370125560933766132969$ 7467168909049024246322586163111533061543629476265022591567289276244918 6669749964134790830864803067349198835488601701433176386332698667132106 $050871780296857,1\}\}$.

(50) $7^{338}+1=\{\{2,1\},\{5,2\},\{157,1\},\{677,1\},\{2029,1\}$,

$\{407752988127621883728249622256495347982646900218466489535182883$

7416768123411313591641262155295034377849280758525399540854087640809336 0250980478626569098994746546168036961270222166849897815938877072434498 0705060706604899902744591077986192226629425614564895011566921447432525 $749,1\}\}$.

(51) $7^{366}+1=\{\{2,1\},\{5,2\},\{13,1\},\{181,1\},\{733,1\},\{4497653,1\},\{133440673,1\}$, $\{390762221068173008631919921610897124445518925029000609050379099$ 8111252226401779950215205406309800002589985110535439833300300040167758 5284645253404090089418337176108494618127999367803527571223435941603508 6597322276361006332381471031593590341976471459531227362658010403667052 $68266736460993,1\}\}$.

(52) $7^{380}+1=\{\{2,1\},\{41,1\},\{761,1\},\{1201,1\},\{44118001,1\},\{700197881,1\}$, 
$\{1081419961,1\},\{810221830361,1\},\{1295161807633,1\}$,

$\{1299254811081649,1\},\{135214434686939513,1\}$,

$\{297192146530040983693236598002549974703128082980666561064628938$

7688457678285916722066405393413108681418760467844887584362190496307223

5916371312472660093608762961299121460113543772724495648394354130061611

$13200696008671657840393102481,1\}\}$.

$(53) 7^{386}+1=\{\{2,1\},\{5,2\},\{773,1\},\{64849,1\}$,

$\{378881050637,1\},\{286400155926593,1\},\{18154096793869693,1\}$,

$\{326843522221684320050996089287320622150668420701127273038700122$

8915639100950331605521558758484764326707629228013434160355745791011266

4124655956826479422504201363921551366051616192722428756882747021378097

1334031235820085526429366154645695884433630898987017567189948729018474 $93,1\}\}$.

$(54) 7^{398}+1=\{\{2,1\},\{5,2\},\{797,1\}$,

$\{560520628036828456417819095325154111233326440150414148124558629$

3065521538235178296271623529407150041263923149234540437945996246647924

7503510046204313557670197137257954048530336296807243274052846674457241

7423884852370768058674561357111608662871466553010200458029522099401937 $82792456728304261958421492680816234265743216657413256205317,1\}\}$.

(55) $7^{428}+1=\{\{2,1\},\{857,1\},\{1201,1\}$,

$\{244572318786606967144121561158907356389231395610027178755620501$

4777370755484140090779011652213145433927986440110821179599566987261005

0466643353078984633268486302517704713837561253234444886288015634933426

9611970773719241278888182191144959061509404114062833622072033294204343

2619201332389489739745040068652346074425674358480342429631432496472451

$2114681787593,1\}\}$.

$(56) 7^{464}+1=\{\{2,1\},\{353,1\},\{929,1\},\{47072139617,1\}$,

$\{432420438051647977193741371406724027589259912090358365754932041$

5255451813094318046176134766598442928590290097182421224440560803370609

3251354236228756081115960401604718398433166114295760494480535808961567

3683554981664401412712204660392381954291859345755721113198843046591754

0372517964946814117933615906401999103544722358212913389126745939256755

$412252209508238705859260638780769,1\}\}$.

(57) $7^{468}+1=\{\{2,1\},\{73,1\},\{193,1\},\{313,1\},\{409,1\},\{937,1\},\{1201,1\},\{8737,1\}$,

$\{42409,1\},\{45553,1\},\{137089,1\},\{85094881,1\},\{32952799801,1\}$,

$\{121698886045196705760022198997936443485344085899528665468062736$

1263263437964255878546512118074406630531197320214663003523933411203614

3193629649201198461799924510817091399701023266014269903285898559022313

9715857391725226376224238344876635979499920774927721951857244336608874

7212723780483065500973717886536777661957750101614066187049436994926328

$1,1\}\}$.

(58) $7^{470}+1=\{\{2,1\},\{5,3\},\{281,1\},\{941,1\},\{3761,1\},\{4021,1\},\{95881,1\}$,

$\{4574857237,1\},\{148625151254595761,1\}$,

$\{240992228467773695219512658281902639556218539586107388693398583$

6005802637755693567049253766721490165521646674013071415790091204716558

3297561760273066063742738085354311145303873787486406987904751484142175

2309667234718928548466127813963723254093376770767286377558278342576044

2939097169415277084121939639651127814439182464994009300592078804427001

$29197241,1\}\}$.

$(59) 7^{498}+1=\{\{2,1\},\{5,2\},\{13,1\},\{181,1\},\{997,1\},\{1077673,1\}$,

$\{571544217341103656799649059690394694778933342803760590061774352$ 
6075595972156196240857472998444961169839581838344803041539749821209293 3674844904422952862337955372165667849404912458039251079138702444564196 5432561201230632566257457026684864301295041156572662235973961484578904 9031569952403070878080320707570403298351496983780683939254080749570323 $8780839368294569580164678608794005643734312625492103006844629493,1\}\}$.

$(60) 7^{506}+1=\{\{2,1\},\{5,2\},\{661,1\},\{1013,1\},\{1409,1\},\{3037,1\},\{26681,1\},\{173053,1\}$, $\{649981,1\},\{15513961,1\},\{83960385389,1\}$, $\{743691019606182795106905681637881393793033662532562296126376555$ 1610973660854319923976766230611889336008246241030630446416382691450144 7053990439443742113783919435105338376907343716117293534455632124927756 6841412134228766697356883353409623549357269625171979269795489906360869 8241234316260510758429795677117790697681047603096968969957910260915461 $5055015042543698236092762498976335241,1\}\}$.

$(61) 7^{524}+1=\{\{2,1\},\{1049,1\},\{1201,1\},\{63929,1\},\{8306449,1\},\{183191479867795697,1\}$, $\{276697917901086773801604134053666414102778614273439509365223961$ 2836002199569711683699520371118976814342035357891832897719576179414832 6803950887027924851652369800607549871857865395875005228447390694399070 5162645529078030218068323821452037526128681733290880995526449244134605 0165899529462157566088421740109800960501386899505915139227519231761090 $83329980575909135561350958171297849112045686793632260214720938577,1\}\}$.

$(62) 7^{534}+1=\{\{2,1\},\{5,2\},\{13,1\},\{181,1\},\{1069,1\},\{2137,1\},\{1154656453,1\}$, $\{61734284877458883038930187984630789511072449111060326898669770$ 3966187229860896854353144530707253120268528533455441225623951688026919 1903551352735441980853051426229640441268423914516986301794331414520246 5615711546305426746207699379312487541120270090919501704082489405763833 2453521865694037699244648921847645655083995040426846341885001699296741 2957045094702281113131125660186996135076585565695621229094386267984835 $8838660433228883721,1\}\}$.

(63) $7^{548}+1=\{\{2,1\},\{1097,1\},\{1201,1\},\{1384249,1\},\{56779677401,1\},\{3885538791193,1\}$, $\{161468536478521209631397377810414069933660635009246965996491189$ 0910038631971775428038603752556615673874564609815708431079172986471838 6381596762319101105858014315639239616774317149468978463381942297386449 1091460580999640371704003399543367777192017966195705197052094795386959 1218028508962895536411029907119047594364077087363068520781660975077340 6823884319994704883796025506301444311196333282587375785955363021573487 $341884219626769,1\}\}$.

(64) $7^{554}+1=\{\{2,1\},\{5,2\},\{1109,1\}$,

$\{275684670287677801721447274913248486262516103861123516308456547$

4782139968524965006530354658458567611576378105861992799147922745435972 0242986855823223733537154087742679841820940216328778327975820961715493 7288557893803527800388066955283410889750491815713541492814002988139111 0380454919335107873412451751502526945083180410171837609849138158682311 3834130189985657412418101646802077246686728398162582361317879105644583 $965038928858205260745858219204121675386640725077853,1\}\}$.

(65) $7^{590}+1=\{\{2,1\},\{5,3\},\{281,1\},\{1181,1\},\{4021,1\},\{149153,1\},\{165437,1\},\{1024477,1\}$, $\{109508800175101,1\},\{551659699804141,1\},\{1084026572852341,1\}$, $\{733983419866121120992853909777483206676046590167917120040528249$ 7556950634485161659210044658724146139462197411174468025069076024750769 3932278578399696036890464615491499564353959216233003356023520246302241 0079033498261465922786762264994404769710202556208182061978590841348095 8686406572210101817502685735684044766224196493831420561582898904621545 
6546053425975466843501725868541335723810900646113919221578038876736583 $39382021776177,1\}\}$.

(66) $7^{596}+1=\{\{2,1\},\{1193,1\},\{1201,1\},\{100129,1\},\{36688884217,1\}$,

$\{453027448666561066192949074650688831446927838321432205913446452$

1052285907948405276236148580156367081134369731233617551519032512663500

9455788681671187756771863515750141274506976497581312523218062105130447 6536636772811799072607571118572036789959205633713311080143212762789781 1508309077875587560828009309972942111119660758638960799128869905879046 03959788325732471746417601773988568883280185183393279607885477324739944 6059390194207959948821293859807721896637254324752885877763818281181249, $1\}\}$.

(67) $7^{608}+1=\{\{2,1\},\{1217,1\},\{908353,1\},\{7699649,1\},\{134818753,1\},\{531968664833,1\}$, $\{540661960800636410432261551798363257159976259191748263657563339$ 5531653656738789246658064465022160156523581669863498255243263043708227 2776044125553080762567255575776589990420981572380304681760760519755142 4608248222330406066611968883209902252487012128531362243702190524967488 8976493633649972717459665920551510708403634732483706548075846779697261 1101356683857323859149858972078441217767440741420284226491564441136200 $10884782039371137873745904183803807089148482162751260286789747201,1\}\}$.

$(68) 7^{618}+1=\{\{2,1\},\{5,2\},\{13,1\},\{181,1\},\{1237,1\},\{2473,1\},\{3709,1\},\{51913,1\}$, $\{115361,1\},\{924529,1\},\{38774969,1\},\{122087137,1\}$, $\{53292869106059277827026427507910346281899738412481515821255942$ 9293557699165214976173550788657447825385987773059461116768206375561031 0906839510696272160596553999067783980708136062793800095569166420214027 0956125821895410854617961031798812631173420392873119988599273741263989 9815688482478073378288716364498711199809974260518034697147008739354336 5774316442052905580906622984850211287014976842109585124283191883524462 $0250148311559458689911059797296679647085043827479426931073850137,1\}\}$.

(69) $7^{624}+1=\{\{2,1\},\{97,1\},\{353,1\},\{1249,1\},\{4993,1\},\{3307201,1\},\{41237249,1\}$, $\{104837857,1\},\{47072139617,1\},\{1409781090433,1\}$,

$\{54136789659630890129797456581264194990354809295313025476347593$ 0876818333931109796396329560841324257560720229181632093393454793073640 0567728487323811054377024406250294650621131899795196819330431731232506 3697972114707947175565511204474449341129130420042674327530175523464533 4581403907275134879799343635006048686648469889107334645549011811755633 0469866720718599019809854108611096275811332242400805802033798204126583 $39303643829195774735517205430416292897602969675169668119201,1\}\}$.

(70) $7^{638}+1=\{\{2,1\},\{5,2\},\{233,1\},\{661,1\},\{1277,1\},\{1409,1\},\{1984181,1\}$,

$\{136853089,1\},\{83960385389,1\},\{55716067510309,1\}$, $\{845336231282845839193231315417379311551764282289934245445374003$ 5948764277157248891402529018405712187500813266946407932089802940261544 8079300303267347296063577819916547151659958342730712261327685990748261 3387310660172992106612071349533539791038357880340820368566794519520813 3076697726398665763231906486063232134815427417718687360207496523501693 9025446923125103571780265183812309036247668106290049962853685361906568 2040445985970034766297864595125841073043721041049124805754373371689054 $2149,1\}\}$.

(71) $7^{650}+1=\{\{2,1\},\{5,4\},\{101,1\},\{157,1\},\{281,1\}$, $\{1301,1\},\{4021,1\},\{13001,1\},\{25301,1\},\{134358901,1\}$, $\{159917647633605219301574043888615680683004725711334386186831858$ 2327574700182265918333738395283244292790015993235028081076741688614751 
2604119351725778584068760639824227908938897350500099258222668153868633 4524314361133165194069770767600215223575755160260286992976988693389546 1193347502516675639658581052196573990371599632392863764502396314419657 3725361233066079506280676635089090907195246485743968222889287447444402 6607238051847333614830215680746271746544855195638556772330058235684245 $5910169625330567548519139647670409,1\}\}$.

(72) $7^{680}+1=\{\{2,1\},\{17,2\},\{881,1\},\{1361,1\},\{169553,1\},\{542081,1\},\{2354161,1\}$, $\{173539292561,1\},\{5388077170097,1\},\{49853897949169,1\}$, $\{664001044859779160166480957521739378730361653192287148213517070$ 3101731457401821114876867047922053708439137967566363537398888006051166 1291067988032091394502403720190532574240797476384808048205578456561059 62906326109829257602677340505380637746158983362885566680729114815754381 3397703692087067005345214743887634859863364841156164876173468961133732 3802862640834148351873958567880764170121592197272018509084166657595871 3727913639528631866222316229030555403081152573879670169474714329270825 $4973537383319241108034968081,1\}\}$.

(73) $7^{1068}+1=\{\{2,1\},\{73,1\},\{193,1\},\{409,1\},\{1201,1\},\{2683661857,1\}$, $\{988103554184969480814372452535171515127558675953793259908534715$ 6993889803509846103836387289744340241860992203459584611128386805287422 9548463921663466052112961551029166716843971614632646077460204268094400 9597783806520335927176836962425990492142333193100478687853536791088668 4227385410807561525134885758522089921457143852217518773389405130119595 6873415275998384552748908327684684972200595453417310967500761993071303 8012051858512068072011691907361024969501022641550992235850275122520900 0019787661044182759562583346932911405148692695467876927983003335256912 9302226707177699544810972352592278600473565623148698563999561528428778 2814823488666573644881618152853125505090917646376482173480570668693518 6570771274666610390151981540807856254966814210402953067646498827335321 8042502668574828271919607408558899747431772697766199275730427112812650 $59741517172685945796785744633674435271649156631393,1\}\}$. 